\title{
INVERTING THE LOCAL GEODESIC X-RAY TRANSFORM ON TENSORS
}

\author{
PLAMEN STEFANOV, GUNTHER UHLMANN AND ANDRÁS VASY
}

\begin{abstract}
We prove the local invertibility, up to potential fields, and stability of the geodesic X-ray transform on tensor fields of order 1 and 2 near a strictly convex boundary point, on manifolds with boundary of dimension $n \geq 3$. We also present an inversion formula. Under the condition that the manifold can be foliated with a continuous family of strictly convex surfaces, we prove a global result which also implies a lens rigidity result near such a metric. The class of manifolds satisfying the foliation condition includes manifolds with no focal points, and does not exclude existence of conjugate points.
\end{abstract}

\section{INTRODUCTION}

Let $(M, g)$ be a compact Riemannian manifold with boundary. The X-ray transform of symmetric covector fields of order $m$ is given by

$$
I f(\gamma)=\int\left\langle f(\gamma(t)), \dot{\gamma}^{m}(t)\right\rangle d t
$$

where, in local coordinates, $\left\langle f, v^{m}\right\rangle=f_{i_{1} \ldots i_{m}} v^{i_{1}} \ldots v^{i_{m}}$, and $\gamma$ runs over all (finite length) geodesics with endpoints on $\partial M$. When $m=0$, we integrate functions; when $m=1, f$ is a covector field, in local coordinates, $f_{j} d x^{j}$; when $m=2, f$ is a symmetric 2 -tensor field $f_{i j} d x^{i} d x^{j}$, etc. The problem is of interest by itself but it also appears as a linearization of boundary and lens rigidity problems, see, e.g., [19, 18, 23, 24, 26, 6, 5, 4. Indeed, when $m=0, f$ can be interpreted as the infinitesimal difference of two conformal factors, and when $m=2, f_{i j}$ can be thought of as an infinitesimal difference of two metrics. The $m=1$ problem arises as a linearization of recovery a velocity fields from the time of fly. The $m=4$ problem appears in linearized elasticity.

The problem we study is the invertibility of $I$. It is well known that potential vector fields, i.e., $f$ which are a symmetric differential $d^{s} v$ of a symmetric field of order $m-1$ vanishing on $\partial M$ (when $m \geq 1$ ), are in the kernel of $I$. When $m=0$, there are no potential fields; when $m=1$, potential fields are just ordinary differentials $d v$ of functions vanishing at the boundary; for $m=2$, potential fields are given by $d^{s} v=\frac{1}{2}\left(v_{i, j}+v_{j, i}\right)$, with $v$ one form, $v=0$ on $\partial M$; etc. The natural invertibility question is then whether If $=0$ implies that $f$ is potential; we call that property s-injectivity below.

Date: October 18, 2014.

1991 Mathematics Subject Classification. 53C65, 35R30, 35S05, 53C21.

The authors were partially supported by the National Science Foundation under grant DMS1301646 (P.S.), CMG-1025259 (G.U. and A.V.) and DMS-1265958 (G.U.) and DMS-1068742 and DMS-1361432 (A.V.). 
This problem has been studied extensively for simple manifolds, i.e., when $\partial M$ is strictly convex and any two points are connected by a unique minimizing geodesic smoothly depending on the endpoints. For simple metrics, in case of functions $(m=0)$, uniqueness and a non-sharp stability estimate was established in [13, 12, 2] using the energy method initiated by Mukhometov, and for $m=1$, in [1. Sharp stability follows from [23. The case $m \geq 2$ is harder with less complete results and the $m=2$ one already contains all the difficulties. In two dimensions, uniqueness for simple metrics and $m=2$ has been proven in 21 following the boundary rigidity proof in [16. For any $m$, this was done in [14].

In dimensions $n \geq 3$, the problem still remains open for $m \geq 2$. Under an explicit upper bound of the curvature, uniqueness and a non-sharp stability was proved by Sharafutdinov, see [18, 19] and the references there, using a suitable version of the energy method developed in [15]. Convexity of $\partial M$ is not essential for those kind of results and the curvature assumption can be replaced by an assumption stronger than requiring no conjugate points, see [20, 7]. This still does not answer the uniqueness question for metrics without conjugate points however. The first and the second author proved in 23, 24, using microlocal and analytic microlocal techniques, that for simple metrics, the problem is Fredholm (modulo potential fields) with a finitely dimensional smooth kernel. For analytic simple metrics, there is uniqueness; and in fact, the uniqueness extends to an open and dense set of simple metrics in $C^{k}, k \gg 1$. Moreover, there is a sharp stability $L^{2}(M) \rightarrow H^{1}(\tilde{M})$ estimate for $f \mapsto I^{*} I f$, where $\tilde{M}$ is some extension of $M$, see [22]. We study the $m=2$ case there for simplicity of the exposition but the methods extend to any $m \geq 2$.

The reason why $m \geq 2$ is harder than the $m=1$ and the $m=0$ cases can be seen from the analysis in 23, 24]. When $m=0$, the presence of the boundary $\partial M$ is not essential - we can extend $(M, g)$ to a complete $(\tilde{M}, \tilde{g})$ and just restrict $I$ to functions supported in a fixed compact set. When $f$ is an one-form $(m=1)$, we have to deal with non-uniqueness due to exact one-forms but then the symmetric differential is $d^{s}$ just the ordinary one $d$. When $n \geq 2, d^{s}$ is an elliptic operator but recovery of $d f$ from $d^{s} f$ is not a local operator. One way to deal with the nonuniqueness due to potential fields is to project on solenoidal ones (orthogonal to the potential fields). This involves solving an elliptic boundary value problem and the presence of the boundary $\partial M$ becomes an essential factor. The standard pseudodifferential calculus is not suited naturally to work on manifolds with boundary.

In [25], the first two authors study manifolds with possible conjugate points of dimension $n \geq 3$. The geodesic manifold (when it is a smooth manifold) has dimension $2 n-2$ which exceeds $n$ when $n \geq 3$. We restrict $I$ there to an open set $\Gamma$ of geodesics. Assuming that $\Gamma$ consists of geodesics without conjugate points so that the conormal bundle $\left\{T^{*} \gamma \mid \gamma \in \Gamma\right\}$ covers $T^{*} M \backslash 0$, we show uniqueness and stability for analytic metrics, and moreover for an open and dense set of such metrics. In this case, even though conjugate points are allowed, the analysis is done on the geodesics in $\Gamma$ assumed to have no such points.

A significant progress is done in the recent work [28, where the second and the third author prove the following local result: if $\partial M$ is strictly convex at $p \in \partial M$ and $n \geq 3$, then $I f$, acting on functions $(m=0)$, known for all geodesics close enough to the tangent ones to $\partial M$ at $p$, determine $f$ near $p$ in a stable way. The new idea in 28. was to introduce an artificial boundary near $p$ cutting off a small 
part of $M$ including $p$ and to apply the scattering calculus in the new domain $\Omega_{c}$, treating the artificial boundary as infinity, see Figure 1 . Then $\Omega_{c}$ is small enough, then a suitable "filtered" backprojection operator is not only Fredholm, but also invertible. We use this idea in the present work, as well. The authors used this linear results in a recent work 27] to prove local boundary and lens rigidity near a convex boundary point.

The purpose of this paper is to invert the geodesic X-ray transform $f \mapsto I f$ on one forms and symmetric 2-tensors $(m=1$ and $m=2)$ for $n \geq 3$ near a strictly convex boundary point. We give a local recovery procedure for $f$ on suitable open sets $\Omega \subset M$ from the knowledge of $\operatorname{If}(\gamma)$ for $\Omega$-local geodesics $\gamma$, i.e. $\gamma$ contained in $\Omega$ with endpoints on $\partial M \cap \Omega$. More precisely, there is an obstacle to the inversion explained above: one-forms or tensors which are potential, i.e. of the form $d^{s} v$, where $v$ is scalar or a one-form, vanishing at $\partial M \cap \Omega$, have vanishing integrals along all the geodesics with endpoints there, so one may always add a potential (exact) form or a potential two-tensor to $f$ and obtain the same localized transform $I f$. Our result is thus the local recovery of $f$ from If up to this gauge freedom; in a stable way. Further, under an additional global convex foliation assumption we also give a global counterpart to this result.

We now state our main results more concretely. Let $\rho$ be a local boundary defining function, so that $\rho \geq 0$ in $M$. It is convenient to also consider a manifold without boundary $(\tilde{M}, g)$ extending $M$. First, as in [28], the main local result is obtained for sufficiently small regions $\Omega=\Omega_{c}=\{x \geq 0, \rho \geq 0\}, x=x_{c}$; see Figure 1. Here $x=0$ is an 'artificial boundary' which is strictly concave as viewed from the region $\Omega$ between it and the actual boundary $\partial M$; this (rather than $\partial M$ ) is the boundary that plays a role in the analysis below.

We set this up in the same way as in 28 by considering a function $\tilde{x}$ with strictly concave level sets from the super-level set side for levels $c,|c|<c_{0}$, and letting

$$
x_{c}=\tilde{x}+c, \Omega_{c}=\left\{x_{c} \geq 0, \rho \geq 0\right\} .
$$

(A convenient normalization is that there is a point $p \in \partial M$ such that $\tilde{x}(p)=0$ and such that $d \tilde{x}(p)=-d \rho(p)$; then one can take e.g. $\tilde{x}(z)=-\rho(z)-\epsilon|z-p|^{2}$ for small $\epsilon>0$, which localizes in a lens shaped region near $p$, or indeed $\tilde{x}=-\rho$ which only localizes near $\partial \Omega$.) Here the requirement on $\tilde{x}$ is, if we assume that $M$ is compact, that there is a continuous function $F$ such that $F(0)=0$ and such that

$$
\Omega_{c} \subset\{\tilde{x}<-c+F(c)\},
$$

i.e. as $c \rightarrow 0, \Omega_{c}$ is a thinner and thinner shell in terms of $\tilde{x}$. As in [28], our constructions are uniform in $c$ for $|c|<c_{0}$. We drop the subscript $c$ from $\Omega_{c}$, i.e. simply write $\Omega$, again as in [28, to avoid overburdening the notation.

A weaker version, in terms of function spaces, of the main local theorem, presented in Corollaries 4.17, 4.18, is then the following. The notation here is that local spaces mean that the condition is satisfied on compact subsets of $\Omega \backslash\{x=0\}$, i.e. the conclusions are not stated uniformly up to the artificial boundary (but are uniform up to the original boundary); this is due to our efforts to minimize the analytic and geometric background in the introduction. The dot denotes supported distributions in the sense of Hörmander relative to the actual boundary $\rho=0$, i.e. distributions in $x>0$ (within the extension $\tilde{M}$ ) whose support lies in $\rho \geq 0$, i.e. for $\dot{H}^{1}$, this is the $H_{0}^{1}$ space. 


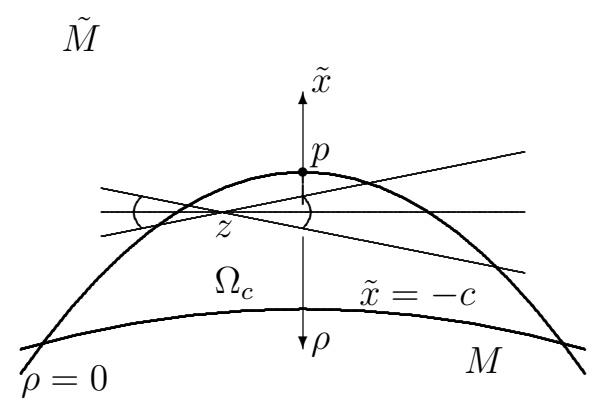

Figure 1. The functions $\rho$ and $\tilde{x}$ when the background is flat space $\tilde{M}$. The intersection of $\rho \geq 0$ and $x_{c}>0$ (where $x_{c}=$ $\tilde{x}+c$, so this is the region $\tilde{x}>-c$ ) is the lens shaped region $O_{p}$. Note that, as viewed from the superlevel sets, thus from $O_{p}, \tilde{x}$ has concave level sets. At the point $z, L$ integrates over geodesics in the indicated small angle. As $z$ moves to the artificial boundary $x_{c}=0$, the angle of this cone shrinks like $C x_{c}$ so that in the limit the geodesics taken into account become tangent to $x_{c}=0$.

Theorem 1.1. (See Corollaries 4.17 4.18,) With $\Omega=\Omega_{c}$ as above, there is $c_{0}>0$ such that for $c \in\left(0, c_{0}\right)$, if $f \in L^{2}(\Omega)$ then $f=u+d^{s} v$, where $v \in \dot{H}_{\mathrm{loc}}^{1}(\Omega \backslash\{x=0\})$, while $u \in L_{\text {loc }}^{2}(\Omega \backslash\{x=0\})$ can be stably determined from If restricted to $\Omega$ local geodesics in the following sense. There is a continuous map If $\mapsto u$, where for $s \geq 0, f$ in $H^{s}(\Omega)$, the $H^{s-1}$ norm of $u$ restricted to any compact subset of $\Omega \backslash\{x=0\}$ is controlled by the $H^{s}$ norm of If restricted to the set of $\Omega$-local geodesics.

Replacing $\Omega_{c}=\{\tilde{x}>-c\} \cap M$ by $\Omega_{\tau, c}=\{\tau>\tilde{x}>-c+\tau\} \cap M$, c can be taken uniform in $\tau$ for $\tau$ in a compact set on which the strict concavity assumption on level sets of $\tilde{x}$ holds.

The uniqueness part of the theorem generalizes Helgason's type of support theorems for tensors fields for analytic metrics [9, 10, 3]. In those works however, analyticity plays a crucial role and the proof is a form of a microlocal analytic continuation. In contrast, no analyticity is assumed here.

As in 28, this theorem can be applied in a manner to obtain a global conclusion. To state this, assume that $\tilde{x}$ is a globally defined function with level sets $\Sigma_{t}$ which are strictly concave from the super-level set for $t \in(-T, 0]$, with $\tilde{x} \leq 0$ on the manifold with boundary $M$. Then we have:

Theorem 1.2. (See Theorem 4.19) Suppose $M$ is compact. Then the geodesic $X$-ray transform is injective and stable modulo potentials on the restriction of oneforms and symmetric 2-tensors $f$ to $\tilde{x}^{-1}((-T, 0])$ in the following sense. For all $\tau>$ $-T$ there is $v \in \dot{H}_{\mathrm{loc}}^{1}\left(\tilde{x}^{-1}((\tau, 0])\right)$ such that $f-d^{s} v \in L_{\mathrm{loc}}^{2}\left(\tilde{x}^{-1}((\tau, 0])\right)$ can be stably recovered from If in the sense that for $s \geq 0$ and $f \in H^{s}$ locally on $\tilde{x}^{-1}((\tau, 0])$, the $H^{s-1}$ norm of $v$ restricted to compact subsets of $\tilde{x}^{-1}((\tau, 0])$ is controlled by the $H^{s}$ norm of If on local geodesics.

Remark 1.3. This theorem, combined with Theorem 2 in [26] (with a minor change - the no-conjugate condition there is only needed to guarantee a stability estimate, 
and we have it in our situation), implies a local, in terms of a perturbation of the metric, lens rigidity uniqueness result near metric satisfying the foliation condition.

Manifolds satisfying the foliation condition include manifolds without focal points 17. Subdomains $M$ of $\mathbf{R}^{n}$ with the metric $c^{-2}(r) d x^{2}, r=|x|$ satisfying the Her-

glotz [8] and Wiechert and Zoeppritz [29] condition $\frac{d}{d r} \frac{r}{c(r)}>0$ on $M$ satisfy it as well since then the Euclidean spheres $|x|=r$ form a strictly convex foliation. Conjugate points in that case may exist, and small perturbations of such metrics satisfy the condition, as well. We can also formulate semi-global results: if we can foliate $M \backslash K$ with $K \subset M$ compact, then we can recover $f$ up to a potential field there in a stable way, with stability degenerating near $\partial M$. This can be considered as a linearized model of the seismology problem for anisotropic speeds of propagation. One such example is metrics $c^{-2}(r) d x^{2}$ (and close to them) for which $\frac{d}{d r} \frac{r}{c(r)}>0$ holds for $a \leq r \leq b$ and $M \subset\{|x| \leq b\}$. Then $f$ can be stably recovered for $|x|>a$ up to a potential field.

Similarly to our work 27, this paper, and its methods, will have applications to the boundary rigidity problem; in this case without the conformal class restriction. This paper is forthcoming.

The plan of the paper is the following. In Section 2 we sketch the idea of the proof, and state the main technical result. In Section 3 we show the ellipticity of the modified version of $L I$, modified by the addition of gauge terms. This essentially proves the main result if one can satisfy the gauge condition. In Section 4 we analyze the gauge condition and complete the proof of our main results.

\section{THE IDEA OF THE PROOF AND THE SCATTERING ALGEBRA}

We now explain the basic ideas of the paper.

The usual approach in dealing with the gauge freedom is to add a gauge condition, which typically, see e.g. the work of the first two authors [24], is of the solenoidal gauge condition form, $\delta_{g}^{s} f=0$, where $\delta_{g}^{s}$ is the adjoint of $d^{s}$ with respect to the Riemannian metric on $M$. Notice that actually the particular choice of the adjoint is irrelevant; once one recovers $f$ in one gauge, one could always express it in terms of another gauge, e.g. in this case relative to a different Riemannian metric.

In order to motivate our gauge condition, we need to recall the method introduced by the last two authors in 28] to analyze the geodesic X-ray transform on functions: the underlying analysis strongly suggests the form the gauge condition should take.

As in 28 we consider an operator $L$ that integrates over geodesics in a small cone at each point, now multiplying with a one form or symmetric 2-tensor, in the direction of the geodesic, mapping (locally defined) functions on the space of geodesics to (locally defined) one forms or tensors. The choice of the operator, or more concretely the angle, plays a big role; we choose it to be comparable to the distance to the artificial boundary, $x=0$. In this case $L I$ ends up being in Melrose's scattering pseudodifferential algebra, at least once conjugated by an exponential weight. (The effect of this weight is that we get exponentially weak estimates as we approach the artificial boundary.) The main analytic problem one faces then is that, corresponding to the gauge freedom mentioned above, $L I$ is not elliptic, unlike in the scalar (function) setting. 
Concretely $L$ is defined as follows. Near $\partial \Omega$, one can use coordinates $(x, y)$, with $x=x_{c}=\tilde{x}+c$ as before, $y$ coordinates on $\partial \Omega$. Correspondingly, elements of $T_{p} M$ can be written as $\lambda \partial_{x}+\eta \partial_{y}$. The unit speed geodesics which are close to being tangential to level sets of $\tilde{x}$ (with the tangential ones being given by $\lambda=0$ ) through a point $p$ can be parameterized by say $(\lambda, \omega)$ (with the actual unit speed being a positive multiple of this) where $\omega$ is unit length with respect to say a Euclidean metric. The concavity of the level sets of $\tilde{x}$, as viewed from the super-level sets, means that $\frac{d^{2}}{d t^{2}} \tilde{x} \circ \gamma$ is bounded below by a positive constant along geodesics in $\Omega_{c}$, as long as $c$ is small, which in turn means that, for sufficiently small $C_{1}>0$, geodesics with $|\lambda|<C_{1} \sqrt{x}$ indeed remain in $x \geq 0$ (as long as they are in $M$ ). Thus, if $I f$ is known along $\Omega$-local geodesics, it is known for geodesics $(x, y, \lambda, \omega)$ in this range. As in 28] we use a smaller range $|\lambda|<C_{2} x$ because of analytic advantages, namely the ability work in the well-behaved scattering algebra. Thus, for $\chi$ smooth, even, non-negative, of compact support, to be specified, in the function case [28] considered the operator

$$
L v(z)=x^{-2} \int \chi(\lambda / x) v\left(\gamma_{x, y, \lambda, \omega}\right) d \lambda d \omega
$$

where $v$ is a (locally, i.e. on $\operatorname{supp} \chi$, defined) function on the space of geodesics, here parameterized by $(x, y, \lambda, \omega)$. (In fact, $L$ had a factor $x^{-1}$ only in [28], with another $x^{-1}$ placed elsewhere; here we simply combine these, as was also done in [27, Section 3]. Also, the particular measure $d \lambda d \omega$ is irrelevant; any smooth positive multiple would work equally well.) In this paper, with $v$ still a locally defined function on the space of geodesics, for one-forms we consider the map $L$

$$
L v(z)=\int \chi(\lambda / x) v\left(\gamma_{x, y, \lambda, \omega}\right) g_{\mathrm{sc}}\left(\lambda \partial_{x}+\omega \partial_{y}\right) d \lambda d \omega
$$

while for 2-tensors

$$
L v(z)=x^{2} \int \chi(\lambda / x) v\left(\gamma_{x, y, \lambda, \omega}\right) g_{\mathrm{sc}}\left(\lambda \partial_{x}+\omega \partial_{y}\right) \otimes g_{\mathrm{sc}}\left(\lambda \partial_{x}+\omega \partial_{y}\right) d \lambda d \omega
$$

so in the two cases $L$ maps into one-forms, resp. symmetric 2-cotensors, where $g_{\mathrm{sc}}$ is a scattering metric used to convert vectors into covectors - this is discussed in detail below.

Since it plays a crucial role even in the setup, by giving the bundles of which our tensors are sections of, as well as the gauge condition, we need to discuss scattering geometry and the scattering pseudodifferential algebra, introduced by Melrose in [11, at least briefly. There is a more thorough discussion in [28, Section 2], though the cotangent bundle, which is crucial here, is suppressed there. Briefly, the scattering pseudodifferential algebra $\Psi_{\mathrm{sc}}^{m, l}(X)$ on a manifold with boundary $X$ is the generalization of the standard pseudodifferential algebra given by quantizations of symbols $a \in S^{m, l}$, i.e. $a \in C^{\infty}\left(\mathbb{R}^{n} \times \mathbb{R}^{n}\right)$ satisfying

$$
\left|D_{z}^{\alpha} D_{\zeta}^{\beta} a(z, \zeta)\right| \leq C_{\alpha \beta}\langle z\rangle^{l-|\alpha|}\langle\zeta\rangle^{-|\beta|}
$$

for all multiindices $\alpha, \beta$ in the same way that on a compact manifold without boundary $\tilde{X}, \Psi^{m}(\tilde{X})$ arises from (localized) pseudodifferential operators on $\mathbb{R}^{n}$ via considering coordinate charts. More precisely, $\mathbb{R}^{n}$ can be compactified to a ball $\overline{\mathbb{R}^{n}}$, by gluing a sphere at infinity, with the gluing done via 'reciprocal polar coordinates'; see [28, Section 2]. One then writes $\Psi_{\mathrm{sc}}^{m, l}\left(\overline{\mathbb{R}^{n}}\right)$ for the quantizations of the symbols 2.3 . Then $\Psi_{\mathrm{sc}}^{m, l}(X)$ is defined by requiring that locally in coordinate 
charts, including charts intersecting with $\partial X$, the algebra arises from $\Psi_{\mathrm{sc}}^{m, l}\left(\overline{\mathbb{R}^{n}}\right)$. (One also has to allow smooth Schwartz kernels on $X \times X$ which are vanishing to infinite order at $\partial(X \times X)$, in analogy with the smooth Schwartz kernels on $\tilde{X} \times \tilde{X}$.) Thus, while the compactification is extremely useful to package information, the reader should keep in mind that ultimately almost all of the analysis reduces to uniform analysis on $\mathbb{R}^{n}$. Since we are working with bundles, we also mention that scattering pseudodifferential operators acting on sections of vector bundles are defined via local trivializations, in which these operators are given by matrices of scalar scattering pseudodifferential operators (i.e. are given by the $\mathbb{R}^{n}$ definition above if in addition these trivializations are made to be coordinate charts), up to the same smooth, infinite order vanishing at $\partial(X \times X)$ Schwartz kernels as in the scalar case.

Concretely, the compactification $\overline{\mathbb{R}^{n}}$, away from $0 \in \mathbb{R}^{n} \subset \overline{\mathbb{R}^{n}}$, is just $[0, \infty)_{x} \times$ $\mathbb{S}_{\omega}^{n-1}$, where the identification with $\mathbb{R}^{n} \backslash\{0\}$ is just the 'inverse polar coordinate' map $(x, \omega) \mapsto x^{-1} \omega$, with $r=x^{-1}$ the standard radial variable. Then a straightforward computation shows that translation invariant vector fields $\partial_{z_{j}}$ on $\mathbb{R}_{z}^{n}$ lift to the compactification (via this identification) to generate, over $C^{\infty}\left(\overline{\mathbb{R}^{n}}\right)$, the Lie algebra $\mathcal{V}_{\mathrm{sc}}\left(\overline{\mathbb{R}^{n}}\right)=x \mathcal{V}_{\mathrm{b}}\left(\overline{\mathbb{R}^{n}}\right)$ of vector fields, where on a manifold with boundary $\mathcal{V}_{\mathrm{b}}(X)$ is the Lie algebra of smooth vector fields tangent to the boundary of $X$. In general, if $x$ is a boundary defining function of $X$, we let $\mathcal{V}_{\mathrm{sc}}(X)=x \mathcal{V}_{\mathrm{b}}(X)$. Then $\Psi_{\mathrm{sc}}^{1,0}(X)$ contains $\mathcal{V}_{\text {sc }}(X)$, corresponding to the analogous inclusion on Euclidean space, and the vector fields in $\Psi_{\mathrm{sc}}^{1,0}(X)$ are essentially the elements of $\mathcal{V}_{\mathrm{sc}}(X)$, after a slight generalization of coefficients (since above $a$ does not have an asymptotic expansion at infinity in $z$, only symbolic estimates; the expansion would correspond to smoothness of the coefficients).

Now, a local basis for $\mathcal{V}_{\mathrm{sc}}(X)$, in a coordinate chart $\left(x, y_{1}, \ldots, y_{n-1}\right)$, is

$$
x^{2} \partial_{x}, x \partial_{y_{1}}, \ldots, x \partial_{y_{n-1}}
$$

directly from the definition, i.e. $V \in \mathcal{V}_{\mathrm{sc}}(X)$ means exactly that locally, on $U \subset X$

$$
V=a_{0}\left(x^{2} \partial_{x}\right)+\sum a_{j}\left(x \partial_{y_{j}}\right), a_{j} \in C^{\infty}(U) .
$$

This gives that elements of $\mathcal{V}_{\mathrm{sc}}(X)$ are exactly smooth sections of a vector bundle, ${ }^{\text {sc }} T X$, with local basis $x^{2} \partial_{x}, x \partial_{y_{1}}, \ldots, x \partial_{y_{n-1}}$. In the case of $X=\overline{\mathbb{R}^{n}}$, this simply means that one is using the local basis $x^{2} \partial_{x}=-\partial_{r}, x \partial_{y_{j}}=r^{-1} \partial_{\omega_{j}}$, where the $\omega_{j}$ are local coordinates on the sphere. An equivalent global basis is just $\partial_{z_{j}}, j=1, \ldots, n$, i.e. ${ }^{\mathrm{sc}} T \overline{\mathbb{R}^{n}}=\overline{\mathbb{R}_{z}^{n}} \times \mathbb{R}^{n}$ is a trivial bundle with this identification.

The dual bundle ${ }^{\mathrm{sc}} T^{*} X$ of ${ }^{\mathrm{sc}} T X$ correspondingly has a local basis $\frac{d x}{x^{2}}, \frac{d y_{1}}{x}, \ldots, \frac{d y_{n-1}}{x}$, which in case of $X=\overline{\mathbb{R}^{n}}$ becomes $-d r, r d \omega_{j}$, with local coordinates $\omega_{j}$ on the sphere. A global version is given by using the basis $d z_{j}$, with covectors written as $\sum \zeta_{j} d z_{j}$; thus ${ }^{\mathrm{sc}} T^{*} \overline{\mathbb{R}^{n}}=\overline{\mathbb{R}_{z}^{n}} \times \mathbb{R}_{\zeta}^{n}$; this is exactly the same notation as in the description of the symbol class $(2.3)$, i.e. one should think of this class as living on ${ }^{\mathrm{sc}} T^{*} \overline{\mathbb{R}^{n}}$. Thus, smooth scattering one-forms on $\overline{\mathbb{R}^{n}}$, i.e. sections of ${ }^{\mathrm{sc}} T^{*} \overline{\mathbb{R}^{n}}$, are simply smooth one-forms on $\mathbb{R}^{n}$ with an expansion at infinity. Similar statements apply to natural bundles, such as the higher degree differential forms ${ }^{\mathrm{sc}} \Lambda^{k} X$, as well as symmetric tensors, such as $\operatorname{Sym}^{2 \mathrm{sc}} T^{*} X$. The latter give rise to scattering metrics $g_{\mathrm{sc}}$, which are positive definite inner products on the fibers of ${ }^{\mathrm{sc}} T X$ (i.e. positive definite sections of $\operatorname{Sym}^{2 \mathrm{sc}} T^{*} X$ ) of the form $g_{\mathrm{sc}}=x^{-4} d x^{2}+x^{-2} \tilde{h}, \tilde{h}$ a standard smooth 2-cotensor on $X$ (i.e. a section of $\operatorname{Sym}^{2} T^{*} X$ ). For instance, one 
can take, in a product decomposition near $\partial X, g_{\mathrm{sc}}=x^{-4} d x^{2}+x^{-2} h, h$ a metric on the level sets of $x$.

The principal symbol of a pseudodifferential operator is the equivalence class of $a$ as in 2.3 modulo $S^{m-1, l-1}$, i.e. modulo additional decay both in $z$ and in $\zeta$ on $\mathbb{R}^{n} \times \mathbb{R}^{n}$. In particular, full ellipticity is ellipticity in this sense, modulo $S^{m-1, l-1}$, i.e. for a scalar operator lower bounds $|a(z, \zeta)| \geq c\langle z\rangle^{l}\langle\zeta\rangle^{m}$ for $|z|+|\zeta|>R$, where $R$ is suitably large. This contrasts with (uniform) ellipticity in the standard sense, which is a similar lower bound, but only for $|\zeta|>R$. Fully elliptic operators are Fredholm between the appropriate Sobolev spaces $H_{\mathrm{sc}}^{s, r}(X)$ corresponding to the scattering structure, see [28, Section 2]; full ellipticity is needed for this (as shown e.g. by taking $\Delta-1$ on $\mathbb{R}^{n}, \Delta$ the flat positive Laplacian). If $a$ is matrix valued, ellipticity can be stated as invertibility for large $(z, \zeta)$, together with upper bounds for the inverse: $\left|a(z, \zeta)^{-1}\right| \leq c^{-1}\langle z\rangle^{-l}\langle\zeta\rangle^{-m}$; this coincides with the above definition for scalars.

We mention also that the exterior derivative $d \in \operatorname{Diff}_{\mathrm{sc}}^{1}\left(X ;{ }^{\mathrm{sc}} \Lambda^{k},{ }^{\mathrm{sc}} \Lambda^{k+1}\right)$ for all $k$. Explicitly, for $k=0$, in local coordinates, this is the statement that

$$
d f=\left(\partial_{x} f\right) d x+\sum_{j}\left(\partial_{y_{j}} f\right) d y_{j}=\left(x^{2} \partial_{x} f\right) \frac{d x}{x^{2}}+\sum_{j}\left(x \partial_{y_{j}}\right) \frac{d y_{j}}{x}
$$

with $x^{2} \partial_{x}, x \partial_{y_{j}} \in \operatorname{Diff}_{\mathrm{sc}}^{1}(X)$, while $\frac{d x}{x^{2}}, \frac{d y_{j}}{x}$ are smooth sections of ${ }^{\mathrm{sc}} T^{*} X$ (locally, where this formula makes sense). Such a computation also shows that the principal symbol, in both senses, of $d$, at any point $\xi \frac{d x}{x^{2}}+\sum_{j} \eta_{j} \frac{d y_{j}}{x}$, is wedge product with $\xi \frac{d x}{x^{2}}+\sum_{j} \eta_{j} \frac{d y_{j}}{x}$. A similar computation shows that the gradient with respect to a scattering metric $g_{\mathrm{sc}}$ is a scattering differential operator (on any of the natural bundles), with principal symbol given by tensor product with $\xi \frac{d x}{x^{2}}+\sum_{j} \eta_{j} \frac{d y_{j}}{x}$, hence so is the symmetric gradient on one forms, with principal symbol given by the symmetrized tensor product with $\xi \frac{d x}{x^{2}}+\sum_{j} \eta_{j} \frac{d y_{j}}{x}$. Note that all of these principal symbols are actually independent of the metric $g_{\mathrm{sc}}$, and $d$ itself is completely independent of any choice of a metric (scattering or otherwise).

If we instead consider the symmetric differential $d^{s}$ with respect to a smooth metric $g$ on $X$, as we are obliged to use in our problem since its image is what is annihilated by the ( $g$-geodesic) X-ray transform $I$, it is a first order differential operator between sections of bundles $T^{*} X$ and $\operatorname{Sym}^{2} T^{*} X$. Writing $d x, d y_{j}$, resp., $d x^{2}$, $d x d y_{j}$ and $d y_{i} d y_{j}$ for the corresponding bases, this means that we have a matrix of first order differential operators. Now, as the standard principal symbol of $d^{s}$ is just tensoring with the covector at which the principal symbol is evaluated, the first order terms are the same, modulo zeroth order terms, as when one considers $d_{g_{\mathrm{sc}}}^{s}$, and in particular they correspond to a scattering differential operator acting between section of ${ }^{\mathrm{sc}} T^{*} X$ and $\mathrm{Sym}^{2 \mathrm{sc}} T^{*} X$. (This can also be checked explicitly using the calculation done below for zeroth order term, but the above is the conceptual reason for this.) On the other hand, with $d x^{2}=d x \otimes d x, d x d y_{i}=\frac{1}{2}\left(d x \otimes d y_{i}+d y_{i} \otimes d x\right)$, etc., these zeroth order terms form a matrix with smooth coefficients in the local basis

$$
d x^{2} \otimes \partial_{x}, d x^{2} \otimes \partial_{y_{j}}, d x d y_{i} \otimes \partial_{x}, d x d y_{i} \otimes \partial_{y_{j}}, d y_{k} d y_{i} \otimes \partial_{x}, d y_{k} d y_{i} \otimes \partial_{y_{j}}
$$


of the homomorphism bundle $\operatorname{hom}\left(T^{*} X, \operatorname{Sym}^{2} T^{*} X\right)$. In terms of the local basis

$$
\begin{aligned}
& \frac{d x^{2}}{x^{4}} \otimes\left(x^{2} \partial_{x}\right), \frac{d x^{2}}{x^{4}} \otimes\left(x \partial_{y_{j}}\right), \frac{d x}{x^{2}} \frac{d y_{i}}{x} \otimes\left(x^{2} \partial_{x}\right), \\
& \frac{d x}{x^{2}} \frac{d y_{i}}{x} \otimes\left(x \partial_{y_{j}}\right), \frac{d y_{k} d y_{i}}{x^{2}} \otimes\left(x^{2} \partial_{x}\right), \frac{d y_{k} d y_{i}}{x^{2}} \otimes\left(x \partial_{y_{j}}\right)
\end{aligned}
$$

of $\operatorname{hom}\left({ }^{\mathrm{sc}} T^{*} X, \operatorname{Sym}^{2 \mathrm{sc}} T^{*} X\right)$, these are all smooth, and vanish at $\partial X$ to order $2,3,1,2,0,1$ respectively, showing that $d^{s} \in \operatorname{Diff}_{\mathrm{sc}}^{1}\left(X,{ }^{\mathrm{sc}} T^{*} X, \operatorname{Sym}^{2 \mathrm{sc}} T^{*} X\right)$, and that the only non-trivial contribution of these zeroth order terms to the principal symbol is via the entry corresponding to $\frac{d y_{k} d y_{i}}{x^{2}} \otimes\left(x^{2} \partial_{x}\right)=d y_{k} d y_{i} \otimes \partial_{x}$, which however is rather arbitrary.

Returning to the choice of gauge, in our case the solenoidal gauge relative to $g$ would not be a good idea: the metric on $M$ is an incomplete metric as viewed at the artificial boundary, and does not interact well with $L I$. We circumvent this difficulty by considering instead the adjoint $\delta^{s}$ relative to a scattering metric, i.e. one of the form $x^{-4} d x^{2}+x^{-2} h, h$ a metric on the level sets of $x$. While $\delta^{s}, d^{s}$ are then scattering differential operators, unfortunately $\delta^{s} d^{s}$ on functions, or one forms, is not fully elliptic in the scattering sense (full ellipticity is needed to guarantee Fredholm properties on Sobolev spaces in a compact setting), with the problem being at finite points of ${ }^{\mathrm{sc}} T^{*} X, X=\{x \geq 0\}$. For instance, in the case of $X$ being the radial compactification of $\mathbb{R}^{n}$, we would be trying to invert the Laplacian on functions or one-forms, which has issues at the 0 -section. However, if we instead use an exponential weight, which already arose when $L I$ was discussed, we can make the resulting operator fully elliptic, and indeed invertible for suitable weights.

Thus, we introduce a Witten-type (in the sense of the Witten Laplacian) solenoidal gauge on the scattering cotangent bundle, ${ }^{\mathrm{sc}} T^{*} X$ or its second symmetric power, $\operatorname{Sym}^{2 \mathrm{sc}} T^{*} X$. Fixing $\digamma>0$, our gauge is

$$
e^{2 \digamma / x} \delta^{s} e^{-2 \digamma / x} f^{s}=0,
$$

or the $e^{-2 \digamma / x}$-solenoidal gauge. (Keep in mind here that $\delta^{s}$ is the adjoint of $d^{s}$ relative to a scattering metric.) We are actually working with

$$
f_{\digamma}=e^{-\digamma / x} f
$$

throughout; in terms of this the gauge is

$$
\delta_{\digamma}^{s} f_{\digamma}^{s}=0, \quad \delta_{\digamma}^{s}=e^{\digamma / x} \delta^{s} e^{-\digamma / x} .
$$

Theorem 2.1. (See Theorem 4.15 for the proof and the formula.) There exists $\digamma_{0}>0$ such that for $\digamma \geq \digamma_{0}$ the following holds.

For $\Omega=\Omega_{c}, c>0$ small, the geodesic $X$-ray transform on $e^{2 \digamma / x}$-solenoidal oneforms and symmetric 2-tensors $f \in e^{\digamma / x} L_{\mathrm{sc}}^{2}(\Omega)$, i.e. ones satisfying $\delta^{s}\left(e^{-2 \digamma / x} f\right)=$ 0 , is injective, with a stability estimate and a reconstruction formula.

In addition, replacing $\Omega_{c}=\{\tilde{x}>-c\} \cap M$ by $\Omega_{\tau, c}=\{\tau>\tilde{x}>-c+\tau\} \cap M$, $c$ can be taken uniform in $\tau$ for $\tau$ in a compact set on which the strict concavity assumption on level sets of $\tilde{x}$ holds.

\section{Ellipticity up to gauge}

With $L$ defined in (2.1)-(2.2), the main analytic points are that, first, $L I$ is (after a suitable exponential conjugation) a scattering pseudodifferential operator of order 
-1 , and second, by choosing an additional appropriate gauge-related summand, this operator $L I$ is elliptic (again, after the exponential conjugation). These results are stated in the next two propositions, with the intermediate Lemma 3.2 describing the gauge related summand.

Proposition 3.1. On one forms, resp. symmetric 2-cotensors, the operators $N_{\digamma}=$ $e^{-\digamma / x} L I e^{\digamma / x}$, lie in

$$
\Psi_{\mathrm{sc}}^{-1,0}\left(X ;{ }^{\mathrm{sc}} T^{*} X,{ }^{\mathrm{sc}} T^{*} X\right), \text { resp. } \Psi_{\mathrm{sc}}^{-1,0}\left(X ; \operatorname{Sym}^{2 \mathrm{sc}} T^{*} X, \operatorname{Sym}^{2 \mathrm{sc}} T^{*} X\right),
$$

for $\digamma>0$.

Proof. The proof of this proposition follows that of the scalar case given in [28, Proposition 3.3] and in a modified version of the scalar case in [27, Proposition 3.2]. For convenience of the reader, we follow the latter proof very closely, except that we do not emphasize the continuity statements in terms of the underlying metric itself, indicating the modifications.

Thus, recall that the map

$$
\Gamma_{+}: S \tilde{M} \times[0, \infty) \rightarrow[\tilde{M} \times \tilde{M} ; \operatorname{diag}], \Gamma_{+}(x, y, \lambda, \omega, t)=\left((x, y), \gamma_{x, y, \lambda, \omega}(t)\right)
$$

is a local diffeomorphism, and similarly for $\Gamma_{-}$in which $(-\infty, 0]$ takes the place of $[0, \infty)$; see the discussion around [28, Equation (3.2)-(3.3)]; indeed this is true for more general curve families. Here $[\tilde{M} \times \tilde{M} ; \operatorname{diag}]$ is the blow-up of $\tilde{M}$ at the diagonal $z=z^{\prime}$, which essentially means the introduction of spherical/polar coordinates, or often more conveniently projective coordinates, about it. Concretely, writing the (local) coordinates from the two factors of $\tilde{M}$ as $\left(z, z^{\prime}\right)$,

$$
z,\left|z-z^{\prime}\right|, \frac{z-z^{\prime}}{\left|z-z^{\prime}\right|}
$$

give (local) coordinates on this space. Since the statement regarding the pseudodifferential property of $L I$ is standard away from $x=0$, we concentrate on the latter region. Correspondingly, in our coordinates $(x, y, \lambda, \omega)$, we write

$$
\left(\gamma_{x, y, \lambda, \omega}(t), \gamma_{x, y, \lambda, \omega}^{\prime}(t)\right)=\left(\mathbf{X}_{x, y, \lambda, \omega}(t), \mathbf{Y}_{x, y, \lambda, \omega}(t), \Lambda_{x, y, \lambda, \omega}^{b}(t), \Omega_{x, y, \lambda, \omega}^{b}(t)\right)
$$

for the lifted geodesic $\gamma_{x, y, \lambda, \omega}(t)$.

Recall from [28, Section 2] that coordinates on Melrose's scattering double space, on which the Schwartz kernels of elements of $\Psi_{\mathrm{sc}}^{s, r}(X)$ are conormal to the diagonal, near the lifted scattering diagonal, are (with $x \geq 0$ )

$$
x, y, X=\frac{x^{\prime}-x}{x^{2}}, Y=\frac{y^{\prime}-y}{x} .
$$

Note that here $X, Y$ are as in 28, around Equation (3.10), not as in 28, Section 2] (where the signs are switched), which means that we need to replace $(\xi, \eta)$ by $(-\xi,-\eta)$ in the Fourier transform when computing principal symbols. Further, it is convenient to write coordinates on $[\tilde{M} \times \tilde{M} ; \operatorname{diag}]$ in the region of interest (see the beginning of the paragraph of Equation (3.10) in [28]), namely (the lift of) $\left|x-x^{\prime}\right|<C\left|y-y^{\prime}\right|$, as

$$
x, y,\left|y-y^{\prime}\right|, \frac{x^{\prime}-x}{\left|y-y^{\prime}\right|}, \frac{y^{\prime}-y}{\left|y-y^{\prime}\right|}
$$

with the norms being Euclidean norms, instead of (3.2); we write $\Gamma_{ \pm}$in terms of these. Note that these are $x, y, x|Y|, \frac{x X}{|Y|}, \hat{Y}$. Moreover, by [28, Equation(3.10)] and 
the subsequent equations, combined also with Equations (3.14)-(3.15) there, $\lambda, \omega, t$ are given in terms of $x, x^{\prime}, y, y^{\prime}$ as

$$
\begin{aligned}
(\Lambda \circ & \left.\Gamma_{ \pm}^{-1}\right)\left(x, y, x|Y|, \frac{x X}{|Y|}, \hat{Y}\right) \\
& =x \frac{\left.X-\alpha\left(x, y, x|Y|, \frac{x X}{|Y|}, \hat{Y}\right)\right)|Y|^{2}}{|Y|}+x^{2} \tilde{\Lambda}_{ \pm}\left(x, y, x|Y|, \frac{x X}{|Y|}, \hat{Y}\right)
\end{aligned}
$$

with $\tilde{\Lambda}_{ \pm}$smooth,

$$
\left(\Omega \circ \Gamma_{ \pm}^{-1}\right)\left(x, y, x|Y|, \frac{x X}{|Y|}, \hat{Y}\right)=\hat{Y}+x|Y| \tilde{\Omega}_{ \pm}\left(x, y, x|Y|, \frac{x X}{|Y|}, \hat{Y}\right)
$$

with $\tilde{\Omega}_{ \pm}$smooth and

$$
\pm\left(T \circ \Gamma_{ \pm}^{-1}\right)\left(x, y, x|Y|, \frac{x X}{|Y|}, \hat{Y}\right)=x|Y|+x^{2}|Y|^{2} \tilde{T}_{ \pm}\left(x, y, x|Y|, \frac{x X}{|Y|}, \hat{Y}\right)
$$

with $\tilde{T}$ smooth.

In particular,

$$
\begin{aligned}
& \left(\Lambda \circ \Gamma_{ \pm}^{-1}\right) \partial_{x}+\left(\Omega \circ \Gamma_{ \pm}^{-1}\right) \partial_{y} \\
& =\left(x \frac{\left.X-\alpha\left(x, y, x|Y|, \frac{x X}{|Y|}, \hat{Y}\right)\right)|Y|^{2}}{|Y|}+x^{2} \tilde{\Lambda}_{ \pm}\left(x, y, x|Y|, \frac{x X}{|Y|}, \hat{Y}\right)\right) \partial_{x} \\
& \quad+\left(\hat{Y}+x|Y| \tilde{\Omega}_{ \pm}\left(x, y, x|Y|, \frac{x X}{|Y|}, \hat{Y}\right)\right) \partial_{y} .
\end{aligned}
$$

Thus, a smooth metric $g_{0}=d x^{2}+h$ applied to this yields

$$
\begin{gathered}
\left(\Lambda \circ \Gamma_{ \pm}^{-1}\right) d x+\left(\Omega \circ \Gamma_{ \pm}^{-1}\right) h\left(\partial_{y}\right)=x\left(x\left(\Lambda \circ \Gamma_{ \pm}^{-1}\right) \frac{d x}{x^{2}}+\left(\Omega \circ \Gamma_{ \pm}^{-1}\right) \frac{h\left(\partial_{y}\right)}{x}\right) \\
=x\left(x^{2}\left(\frac{\left.X-\alpha\left(x, y, x|Y|, \frac{x X}{|Y|}, \hat{Y}\right)\right)|Y|^{2}}{|Y|}+x \tilde{\Lambda}_{ \pm}\left(x, y, x|Y|, \frac{x X}{|Y|}, \hat{Y}\right)\right) \frac{d x}{x^{2}}\right. \\
\left.+\left(\hat{Y}+x|Y| \tilde{\Omega}_{ \pm}\left(x, y, x|Y|, \frac{x X}{|Y|}, \hat{Y}\right)\right) \frac{h\left(\partial_{y}\right)}{x}\right),
\end{gathered}
$$

while so $g_{\mathrm{sc}}$ applied to this yields

$$
\begin{aligned}
& x^{-1}\left(x^{-1}\left(\Lambda \circ \Gamma_{ \pm}^{-1}\right) \frac{d x}{x^{2}}+\left(\Omega \circ \Gamma_{ \pm}^{-1}\right) \frac{h\left(\partial_{y}\right)}{x}\right) \\
& =x^{-1}\left(\left(\frac{\left.X-\alpha\left(x, y, x|Y|, \frac{x X}{|Y|}, \hat{Y}\right)\right)|Y|^{2}}{|Y|}+x \tilde{\Lambda}_{ \pm}\left(x, y, x|Y|, \frac{x X}{|Y|}, \hat{Y}\right)\right) \frac{d x}{x^{2}}\right. \\
& \left.\quad+\left(\hat{Y}+x|Y| \tilde{\Omega}_{ \pm}\left(x, y, x|Y|, \frac{x X}{|Y|}, \hat{Y}\right)\right) \frac{h\left(\partial_{y}\right)}{x}\right) .
\end{aligned}
$$

Notice that on the right hand side of (3.4) the singular factor of $x^{-1}$ in front of $\frac{d x}{x^{2}}$ disappears due to the factor $x$ in $\Lambda$, while on the right hand side of 3.3 correspondingly $\frac{d x}{x^{2}}$ has a vanishing factor $x^{2}$. This means, as we see below, that the $\frac{d x}{x^{2}}$ component behaves trivially at the level of the boundary principal symbol of the operator $N_{\digamma, 0}$ defined like $N_{\digamma}$ but with $g_{0}$ in place of $g_{\mathrm{sc}}$, so in fact one can never have full ellipticity in this case; this is the reason we must use $g_{\mathrm{sc}}$ in the definition of $N_{\digamma}$. 
One also needs to have $\Lambda_{x, y, \lambda, \omega}^{b}(t), \Omega_{x, y, \lambda, \omega}^{b}(t)$ evaluated at $\left(x^{\prime}, y^{\prime}\right)$, since this is the tangent vector $\lambda^{\prime} \partial x^{\prime}+\omega^{\prime} \partial_{y^{\prime}}$ with which our tensors are contracted as they are being integrated along the geodesic. In order to compute this efficiently, we recall from [28, Equation (3.14)] that

$$
x^{\prime}=x+\lambda t+\alpha(x, y, \lambda, \omega) t^{2}+O\left(t^{3}\right), y^{\prime}=y+\omega t+O\left(t^{2}\right),
$$

with the $O\left(t^{3}\right)$, resp. $O\left(t^{2}\right)$ terms having smooth coefficients in terms of $(x, y, \lambda, \omega)$. Correspondingly,

$$
\lambda^{\prime}=\frac{d x}{d t}=\lambda+2 \alpha(x, y, \lambda, \omega) t+O\left(t^{2}\right), \omega^{\prime}=\frac{d y}{d t}=\omega+O(t) .
$$

This gives that in terms of $x, y, x^{\prime}, y^{\prime}, \lambda^{\prime}$ is given by

$$
\Lambda^{\prime} \circ \Gamma_{ \pm}^{-1}=\Lambda \circ \Gamma_{ \pm}^{-1}+2 \alpha\left(x, y, \Lambda \circ \Gamma_{ \pm}^{-1}, \Omega \circ \Gamma_{ \pm}^{-1}\right)\left(T \circ \Gamma_{ \pm}^{-1}\right)+\left(T \circ \Gamma_{ \pm}^{-1}\right)^{2} \tilde{\Lambda}^{\prime} \circ \Gamma_{ \pm}^{-1},
$$

with $\tilde{\Lambda}^{\prime}$ smooth in terms of $x, y, \Lambda \circ \Gamma_{ \pm}^{-1}, \Omega \circ \Gamma_{ \pm}^{-1}, T \circ \Gamma_{ \pm}^{-1}$. Substituting these in yields

$$
\begin{aligned}
\Lambda^{\prime} \circ \Gamma_{ \pm}^{-1} & =x \frac{X-\alpha\left(x, y, x|Y|, \frac{x X}{|Y|}, \hat{Y}\right)|Y|^{2}}{|Y|}+2 x|Y| \alpha\left(x, y, x|Y|, \frac{x X}{|Y|}, \hat{Y}\right) \\
+x^{2}|Y|^{2} \tilde{\Lambda}^{\prime}\left(x, y, x|Y|, \frac{x X}{|Y|}, \hat{Y}\right) & \\
= & x \frac{X+\alpha\left(x, y, x|Y|, \frac{x X}{|Y|}, \hat{Y}\right)|Y|^{2}}{|Y|}+x^{2}|Y|^{2} \tilde{\Lambda}^{\prime}\left(x, y, x|Y|, \frac{x X}{|Y|}, \hat{Y}\right)
\end{aligned}
$$

while

$$
\Omega^{\prime} \circ \Gamma_{ \pm}^{-1}=\hat{Y}+x \tilde{\Omega}^{\prime}\left(x, y, x|Y|, \frac{x X}{|Y|}, \hat{Y}\right)
$$

Correspondingly,

$$
\begin{aligned}
\left(\Lambda^{\prime} \circ \Gamma_{ \pm}^{-1}\right) \partial_{x}+\left(\Omega^{\prime} \circ \Gamma_{ \pm}^{-1}\right) \partial_{y}=x^{-1}\left(x^{-1}\left(\Lambda^{\prime} \circ \Gamma_{ \pm}^{-1}\right) x^{2} \partial_{x}+\left(\Omega^{\prime} \circ \Gamma_{ \pm}^{-1}\right) x \partial_{y}\right) \\
=x^{-1}\left(\left(\frac{X+\alpha\left(x, y, x|Y|, \frac{x X}{|Y|}, \hat{Y}\right)|Y|^{2}}{|Y|}+x|Y|^{2} \tilde{\Lambda}_{ \pm}^{\prime}\left(x, y, x|Y|, \frac{x X}{|Y|}, \hat{Y}\right)\right) x^{2} \partial_{x}\right. \\
\left.\quad+\left(\hat{Y}+x|Y| \tilde{\Omega}_{ \pm}^{\prime}\left(x, y, x|Y|, \frac{x X}{|Y|}, \hat{Y}\right)\right) x \partial_{y}\right) .
\end{aligned}
$$

Then, similarly, near the boundary as in [28, Equation (3.13)], one obtains the Schwartz kernel of $N_{\digamma}$ on one forms:

(3.5)

$$
\begin{gathered}
K^{b}(x, y, X, Y) \\
=\sum_{ \pm} e^{-\digamma X /(1+x X)} \chi\left(\frac{X-\alpha\left(x, y, x|Y|, \frac{x X}{|Y|}, \hat{Y}\right)|Y|^{2}}{|Y|}+x \tilde{\Lambda}_{ \pm}\left(x, y, x|Y|, \frac{x|X|}{|Y|}, \hat{Y}\right)\right) \\
\left(x^{-1}\left(\Lambda \circ \Gamma_{ \pm}^{-1}\right) \frac{d x}{x^{2}}+\left(\Omega \circ \Gamma_{ \pm}^{-1}\right) \frac{h\left(\partial_{y}\right)}{x}\right)\left(x^{-1}\left(\Lambda^{\prime} \circ \Gamma_{ \pm}^{-1}\right) x^{2} \partial_{x}+\left(\Omega^{\prime} \circ \Gamma_{ \pm}^{-1}\right) x \partial_{y}\right) \\
|Y|^{-n+1} J_{ \pm}\left(x, y, \frac{X}{|Y|},|Y|, \hat{Y}\right),
\end{gathered}
$$


with the density factor $J$ smooth, positive, $=1$ at $x=0$; there is a similar formula for 2-tensors. Note that the factor $x^{-1}$ in (3.4), as well as another $x^{-1}$ from writing

$$
\left(\Lambda^{\prime} \circ \Gamma_{ \pm}^{-1}\right) \partial_{x}+\left(\Omega^{\prime} \circ \Gamma_{ \pm}^{-1}\right) \partial_{y}=x^{-1}\left(x^{-1}\left(\Lambda^{\prime} \circ \Gamma_{ \pm}^{-1}\right) x^{2} \partial_{x}+\left(\Omega^{\prime} \circ \Gamma_{ \pm}^{-1}\right) x \partial_{y}\right)
$$

are absorbed into the definition of $L,(2.1)-(2.2)$, hence the different powers $(-2$ for functions, 0 on one-forms, 2 for 2-cotensors) appearing there. Here

$$
x, y,|Y|, \frac{X}{|Y|}, \hat{Y}
$$

are valid coordinates on the blow-up of the scattering diagonal in $|Y|>\epsilon|X|, \epsilon>0$, which is the case automatically on the support of the kernel due to the argument of $\chi$, cf. the discussion after [28, Equation(3.12)], so the argument of $\chi$ is smooth on this blown up space. In addition, due to the order $x$ vanishing of $\Lambda$,

$$
x^{-1}\left(\Lambda \circ \Gamma_{ \pm}^{-1}\right) \frac{d x}{x^{2}}+\left(\Omega \circ \Gamma_{ \pm}^{-1}\right) \frac{h\left(\partial_{y}\right)}{x}, \text { resp. } x^{-1}\left(\Lambda \circ \Gamma_{ \pm}^{-1}\right) x^{2} \partial_{x}+\left(\Omega \circ \Gamma_{ \pm}^{-1}\right) x \partial_{y}
$$

are smooth sections of ${ }^{\mathrm{sc}} T^{*} X$, resp. ${ }^{\mathrm{sc}} T X$, pulled back from the left, resp. right, factor of $X^{2}$, thus their product defines a smooth section of the endomorphism bundle of ${ }^{\mathrm{sc}} T^{*} X$.

Since this homomorphism factor is the only difference from [28, Proposition 3.3], and we have shown its smoothness properties as a bundle endomorphism, this proves the proposition as in [28, Proposition 3.3].

If we defined $N_{\digamma, 0}$ as $N_{\digamma}$ but using a smooth metric $g_{0}$ in place of $g_{\mathrm{sc}}$, we would have the Schwartz kernel

(3.6)

$$
\begin{gathered}
K_{0}^{b}(x, y, X, Y) \\
=\sum_{ \pm} e^{-\digamma X /(1+x X)} \chi\left(\frac{X-\alpha\left(x, y, x|Y|, \frac{x X}{|Y|}, \hat{Y}\right)|Y|^{2}}{|Y|}+x \tilde{\Lambda}_{ \pm}\left(x, y, x|Y|, \frac{x|X|}{|Y|}, \hat{Y}\right)\right) \\
\left(x\left(\Lambda \circ \Gamma_{ \pm}^{-1}\right) \frac{d x}{x^{2}}+\left(\Omega \circ \Gamma_{ \pm}^{-1}\right) \frac{h\left(\partial_{y}\right)}{x}\right)\left(x^{-1}\left(\Lambda^{\prime} \circ \Gamma_{ \pm}^{-1}\right) x^{2} \partial_{x}+\left(\Omega^{\prime} \circ \Gamma_{ \pm}^{-1}\right) x \partial_{y}\right) \\
|Y|^{-n+1} J_{ \pm}\left(x, y, \frac{X}{|Y|},|Y|, \hat{Y}\right)
\end{gathered}
$$

and

$$
x\left(\Lambda \circ \Gamma_{ \pm}^{-1}\right) \frac{d x}{x^{2}}+\left(\Omega \circ \Gamma_{ \pm}^{-1}\right) \frac{h\left(\partial_{y}\right)}{x}, \operatorname{resp} . x^{-1}\left(\Lambda \circ \Gamma_{ \pm}^{-1}\right) x^{2} \partial_{x}+\left(\Omega \circ \Gamma_{ \pm}^{-1}\right) x \partial_{y}
$$

are again smooth sections of ${ }^{\mathrm{sc}} T^{*} X$, resp. ${ }^{\text {sc }} T X$, pulled back from the left, resp. right, factor of $X^{2}$, but, as pointed out earlier, with the coefficient of $\frac{d x}{x^{2}}$ vanishing, thus eliminating the possibility of ellipticity in this case.

Before proceeding, we compute the principal symbol of the gauge term $d_{\digamma}^{s} \delta_{\digamma}^{s}$. For this recall that $d_{\digamma}^{s}=e^{-\digamma / x} d^{s} e^{\digamma / x}$, with $d^{s}$ defined using the background metric $g$ (on one forms; the metric is irrelevant for functions), and $\delta_{\digamma}^{s}$ is its adjoint with respect to the scattering metric $g_{\mathrm{sc}}$ (not $g$ ). In order to give the principal symbols, we use the basis

$$
\frac{d x}{x^{2}}, \frac{d y}{x}
$$


for one forms, with $\frac{d y}{x}$ understood as a short hand for $\frac{d y_{1}}{x}, \ldots, \frac{d y_{n-1}}{x}$, while for 2-tensors, we use a decomposition

$$
\frac{d x}{x^{2}} \otimes \frac{d x}{x^{2}}, \frac{d x}{x^{2}} \otimes \frac{d y}{x}, \frac{d y}{x} \otimes \frac{d x}{x^{2}}, \frac{d y}{x} \otimes \frac{d y}{x} .
$$

Note that symmetry of a 2-tensor is the statement that the 2 nd and 3rd (block) entries are the same (up to the standard identification), so for symmetric 2-tensors we can also use

$$
\frac{d x}{x^{2}} \otimes_{s} \frac{d x}{x^{2}}, \frac{d x}{x^{2}} \otimes_{s} \frac{d y}{x}, \frac{d y}{x} \otimes_{s} \frac{d y}{x},
$$

where the middle component is the common $\frac{d x}{x^{2}} \otimes \frac{d y}{x}$ and $\frac{d y}{x} \otimes \frac{d x}{x^{2}}$ component.

Lemma 3.2. On one forms, the operator $d_{\digamma}^{s} \delta_{\digamma}^{s} \in \operatorname{Diff}_{\mathrm{sc}}^{2,0}\left(X ;{ }^{\mathrm{sc}} T^{*} X,{ }^{\mathrm{sc}} T^{*} X\right)$ has principal symbol

$$
\left(\begin{array}{c}
\xi+i \digamma \\
\eta \otimes
\end{array}\right)\left(\begin{array}{ll}
\xi-i \digamma & \iota_{\eta}
\end{array}\right)=\left(\begin{array}{cc}
\xi^{2}+\digamma^{2} & (\xi+i \digamma) \iota_{\eta} \\
(\xi-i \digamma) \eta \otimes & \eta \otimes \iota_{\eta}
\end{array}\right) .
$$

On the other hand, on symmetric 2-tensors $d_{\digamma}^{s} \delta_{\digamma}^{s} \in \operatorname{Diff}_{\mathrm{sc}}^{2,0}\left(X ; \operatorname{Sym}^{2 \mathrm{sc}} T^{*} X, \operatorname{Sym}^{2 \mathrm{sc}} T^{*} X\right)$ has principal symbol

$$
\begin{aligned}
& \left(\begin{array}{cc}
\xi+i \digamma & 0 \\
\frac{1}{2} \eta \otimes & \frac{1}{2}(\xi+i \digamma) \\
a & \eta \otimes_{s}
\end{array}\right)\left(\begin{array}{ccc}
\xi-i \digamma & \frac{1}{2} \iota_{\eta} & \langle a, .\rangle \\
0 & \frac{1}{2}(\xi-i \digamma) & \iota_{\eta}^{s}
\end{array}\right) \\
& =\left(\begin{array}{ccc}
\xi^{2}+\digamma^{2} & \frac{1}{2}(\xi+i \digamma) \iota_{\eta} & (\xi+i \digamma)\langle a, .\rangle \\
\frac{1}{2}(\xi-i \digamma) \eta \otimes & \frac{1}{4}(\eta \otimes) \iota_{\eta}+\frac{1}{4}\left(\xi^{2}+\digamma^{2}\right) & \frac{1}{2} \eta \otimes\langle a, .\rangle+\frac{1}{2}(\xi+i \digamma) \iota_{\eta}^{s} \\
(\xi-i \digamma) a & \frac{1}{2} a \iota_{\eta}+\frac{1}{2}(\xi-i \digamma) \eta \otimes & a\langle a, .\rangle+\eta \otimes_{s} \iota_{\eta},
\end{array}\right)
\end{aligned}
$$

where $a$ is a suitable symmetric 2-tensor.

Proof. This is an algebraic symbolic computation, so in particular it can be done pointwise. Since one can arrange that the metric $g_{\mathrm{sc}}$ used to compute adjoints is of the form $x^{-4} d x^{2}+x^{-2} d y^{2}$, where $d y^{2}$ is the flat metric, at the point in question, one can simply use this in the computation. With our coordinates at the point in question, trivializing the inner product, $g_{\mathrm{sc}}$, the inner product on one-forms is given by the matrix

$$
\left(\begin{array}{cc}
1 & 0 \\
0 & \mathrm{Id}
\end{array}\right)
$$

while on 2-tensors by

$$
\left(\begin{array}{cccc}
1 & 0 & 0 & 0 \\
0 & \mathrm{Id} & 0 & 0 \\
0 & 0 & \mathrm{Id} & 0 \\
0 & 0 & 0 & \mathrm{Id}
\end{array}\right) .
$$

First consider one-forms. Recall from Section 2 that the full principal symbol of $d$, in $\operatorname{Diff}_{\mathrm{sc}}^{1}\left(X ; \underline{\mathbb{C}},{ }^{\mathrm{sc}} T^{*} X\right)$, with $\underline{\mathbb{C}}$ the trivial bundle, is, as a map from functions to one-forms,

$$
\left(\begin{array}{c}
\xi \\
\eta \otimes
\end{array}\right)
$$


Thus the symbol of $d_{\digamma}^{s}=e^{-\digamma / x} d^{s} e^{\digamma / x}$, which conjugation effectively replaces $\xi$ by $\xi+i \digamma\left(\right.$ as $\left.e^{-\digamma / x} x^{2} D_{x} e^{\digamma / x}=x^{2} D_{x}+i \digamma\right)$, is

$$
\left(\begin{array}{c}
\xi+i \digamma \\
\eta \otimes
\end{array}\right)
$$

Hence $\delta_{\digamma}^{s}$ has symbol given by the adjoint of that of $d_{\digamma}^{s}$ with respect to the inner product of $g_{\mathrm{sc}}$, which is

$$
\left(\begin{array}{ll}
\xi-i \digamma & \iota
\end{array}\right) .
$$

Thus, the principal symbol of $d_{\digamma}^{s} \delta_{\digamma}^{s}$ is the product,

$$
\left(\begin{array}{cc}
\xi^{2}+\digamma^{2} & (\xi+i \digamma) \iota_{\eta} \\
(\xi-i \digamma) \eta \otimes & \eta \otimes \iota_{\eta}
\end{array}\right)
$$

proving the lemma for one forms.

We now turn to symmetric 2-tensors. Again, recall from Section 2 that the full principal symbol of the gradient relative to $g$, in $\operatorname{Diff}_{\mathrm{sc}}^{1}\left(X ;{ }^{\mathrm{sc}} T^{*} X ;{ }^{\mathrm{sc}} T^{*} X \otimes{ }^{\mathrm{sc}} T^{*} X\right)$, is, as a map from one-forms to 2-tensors (which we write in the four block form as before) is

$$
\left(\begin{array}{cc}
\xi & 0 \\
\eta \otimes & 0 \\
0 & \xi \\
b & \eta \otimes
\end{array}\right)
$$

where $b$ is a 2-tensor on $Y=\partial X$, and thus that of $d^{s}$ (with symmetric 2-tensors considered as a subspace of 2 -tensors) is

$$
\left(\begin{array}{cc}
\xi & 0 \\
\frac{1}{2} \eta \otimes & \frac{1}{2} \xi \\
\frac{1}{2} \eta \otimes & \frac{1}{2} \xi \\
a & \eta \otimes_{s}
\end{array}\right)
$$

with $a$ a symmetric 2 -tensor (the symmetrization of $b$ ). (Notice that $a, b$ only play a role in the principal symbol at the boundary, not in the standard principal symbol, i.e. as $(\xi, \eta) \rightarrow \infty$.) Here $a$ arises due to the treatment of $d^{s}$, which is defined using a standard metric $g$, as an element of $\operatorname{Diff}{ }_{\mathrm{sc}}\left(X ;{ }^{\mathrm{sc}} T^{*} X, \operatorname{Sym}^{2 \mathrm{sc}} T^{*} X\right)$; it is acting on the one-dimensional space $\operatorname{Span}\left\{\frac{d x}{x^{2}}\right\}$ by multiplying the coefficient of $\frac{d x}{x^{2}}$ to produce a symmetric 2-tensor on $Y$. Note that here the lower right block has $(i j k)$ entry (corresponding to the $(i j)$ entry of the symmetric 2-tensor and the $k$ entry of the one-form) given by $\frac{1}{2}\left(\eta_{i} \delta_{j k}+\eta_{j} \delta_{i k}\right)$. Thus the symbol of $d_{\digamma}^{s}=e^{-\digamma / x} d^{s} e^{\digamma / x}$, which conjugation effectively replaces $\xi$ by $\xi+i \digamma$ (as $\left.e^{-\digamma / x} x^{2} D_{x} e^{\digamma / x}=x^{2} D_{x}+i \digamma\right)$, is

$$
\left(\begin{array}{cc}
\xi+i \digamma & 0 \\
\frac{1}{2} \eta \otimes & \frac{1}{2}(\xi+i \digamma) \\
\frac{1}{2} \eta \otimes & \frac{1}{2}(\xi+i \digamma) \\
a & \eta \otimes_{s}
\end{array}\right) .
$$

Thus, $\delta_{\digamma}^{s}$ has symbol given by the adjoint of that of $d_{\digamma}^{s}$ with respect to this inner product, which is

$$
\left(\begin{array}{cccc}
\xi-i \digamma & \frac{1}{2} \iota_{\eta} & \frac{1}{2} \iota_{\eta} & \langle a, .\rangle \\
0 & \frac{1}{2}(\xi-i \digamma) & \frac{1}{2}(\xi-i \digamma) & \iota_{\eta}^{s}
\end{array}\right) .
$$

Here the lower right block has $(\ell i j)$ entry given by $\frac{1}{2}\left(\eta_{i} \delta_{\ell j}+\eta_{j} \delta_{i \ell}\right)$. Here the inner product $\langle a,$.$\rangle as well as \iota_{\eta}$ are with respect to the identity because of the 
trivialization of the inner product; invariantly they with respect to the inner product induced by $h$. Correspondingly, the product, in the more concise notation for symmetric tensors, has the symbol as stated, proving the lemma.

The proof of the next proposition, on ellipticity, relies on the subsequently stated two lemmas, whose proofs in turn take up the rest of this section.

Proposition 3.3. First consider the case of one forms. Let $\digamma>0$. Given $\tilde{\Omega}, a$ neighborhood of $X \cap M=\{x \geq 0, \rho \geq 0\}$ in $X$, for suitable choice of the cutoff $\chi \in C_{c}^{\infty}(\mathbb{R})$ and of $M \in \Psi_{\mathrm{sc}}^{-3,0}(X)$, the operator

$$
A_{\digamma}=N_{\digamma}+d_{\digamma}^{s} M \delta_{\digamma}^{s}, \quad N_{\digamma}=e^{-\digamma / x} L I e^{\digamma / x}, \quad d_{\digamma}^{s}=e^{-\digamma / x} d^{s} e^{\digamma / x},
$$

is elliptic in $\Psi_{\mathrm{sc}}^{-1,0}\left(X ;{ }^{\mathrm{sc}} T^{*} X,{ }^{\mathrm{sc}} T^{*} X\right)$ in $\tilde{\Omega}$.

On the other hand, consider the case of symmetric 2-tensors. Then there exists $\digamma_{0}>0$ such that for $\digamma>\digamma_{0}$ the following holds. Given $\tilde{\Omega}$, a neighborhood of $X \cap M=\{x \geq 0, \rho \geq 0\}$ in $X$, for suitable choice of the cutoff $\chi \in C_{c}^{\infty}(\mathbb{R})$ and of $M \in \Psi_{\mathrm{sc}}^{-3,0}\left(X ;{ }^{\mathrm{sc}} T^{*} X,{ }^{\mathrm{sc}} T^{*} X\right)$, the operator

$$
A_{\digamma}=N_{\digamma}+d_{\digamma}^{s} M \delta_{\digamma}^{s}, \quad N_{\digamma}=e^{-\digamma / x} L I e^{\digamma / x}, \quad d_{\digamma}^{s}=e^{-\digamma / x} d^{s} e^{\digamma / x},
$$

is elliptic in $\Psi_{\mathrm{sc}}^{-1,0}\left(X ; \mathrm{Sym}^{2} \mathrm{sc}^{*} X, \operatorname{Sym}^{2 \mathrm{sc}} T^{*} X\right)$ in $\tilde{\Omega}$.

Proof. The proof of this proposition is straightforward given the two lemmas we prove below. Indeed, as we prove below in Lemma 3.4 provided $\chi \geq 0, \chi(0)>0$, the operator $e^{-\digamma / x} L I e^{\digamma / x}$ has positive definite principal symbol at fiber infinity in the scattering cotangent bundle when restricted to the subspace of ${ }^{\mathrm{sc}} T^{*} X$ or $\mathrm{Sym}^{2 \mathrm{sc}} T^{*} X$ given by the kernel of the symbol of $\delta_{\digamma}^{s}$, where the inner product is that of the scattering metric we consider (with respect to which $\delta^{s}$ is computed); in Lemma 3.5 we show a similar statement for the principal symbol at finite points under the assumption that $\chi$ is sufficiently close, in a suitable sense, to an even positive Gaussian, with the complication that for 2 -tensors we need to assume $\boldsymbol{\digamma}>0$ sufficiently large. Thus, if we add $d_{\digamma}^{s} M \delta_{\digamma}^{s}$ to it, where $M$ has positive principal symbol, and is of the correct order, we obtain an elliptic operator, completing the proof of Proposition 3.3 .

We are thus reduced to proving the two lemmas we used.

Lemma 3.4. Both on one-forms and on symmetric 2-tensors, $N_{\digamma}$ is elliptic at fiber infinity in ${ }^{\mathrm{sc}} T^{*} X$ when restricted to the kernel of the principal symbol of $\delta_{\digamma}^{s}$.

Proof. This is very similar to the scalar setting. With

$$
S=\frac{X-\alpha(\hat{Y})|Y|^{2}}{|Y|}, \hat{Y}=\frac{Y}{|Y|},
$$

the Schwartz kernel of $N_{\digamma}$ at the scattering front face $x=0$ is, by (3.5), given by

$$
e^{-\digamma X}|Y|^{-n+1} \chi(S)\left(\left(S \frac{d x}{x^{2}}+\hat{Y} \cdot \frac{d y}{x}\right)\left((S+2 \alpha|Y|)\left(x^{2} \partial_{x}\right)+\hat{Y} \cdot\left(x \partial_{y}\right)\right)\right)
$$

on one forms, respectively

$$
\begin{aligned}
& e^{-\digamma X}|Y|^{-n+1} \chi(S) \\
& \quad\left(\left(\left(S \frac{d x}{x^{2}}+\hat{Y} \cdot \frac{d y}{x}\right) \otimes\left(\left(S \frac{d x}{x^{2}}+\hat{Y} \cdot \frac{d y}{x}\right)\right)\right)\right) \\
& \quad\left(\left((S+2 \alpha|Y|)\left(x^{2} \partial_{x}\right)+\hat{Y} \cdot\left(x \partial_{y}\right)\right) \otimes\left((S+2 \alpha|Y|)\left(x^{2} \partial_{x}\right)+\hat{Y} \cdot\left(x \partial_{y}\right)\right)\right)
\end{aligned}
$$


on 2-tensors, where $\hat{Y}$ is regarded as a tangent vector which acts on covectors, and where $(S+2 \alpha|Y|)\left(x^{2} \partial_{x}\right)+\hat{Y} \cdot\left(x \partial_{y}\right)$ maps one forms to scalars, thus

$$
\left((S+2 \alpha|Y|)\left(x^{2} \partial_{x}\right)+\hat{Y} \cdot\left(x \partial_{y}\right)\right) \otimes\left((S+2 \alpha|Y|)\left(x^{2} \partial_{x}\right)+\hat{Y} \cdot\left(x \partial_{y}\right)\right)
$$

maps symmetric 2 -tensors to scalars, while $S \frac{d x}{x^{2}}+\hat{Y} \cdot \frac{d y}{x}$ maps scalars to one forms, so

$$
\left(S \frac{d x}{x^{2}}+\hat{Y} \cdot \frac{d y}{x}\right) \otimes\left(S \frac{d x}{x^{2}}+\hat{Y} \cdot \frac{d y}{x}\right)
$$

maps scalars to symmetric 2-tensors. In order to make the notation less confusing, we employ a matrix notation,

$$
\begin{gathered}
\left(S \frac{d x}{x^{2}}+\hat{Y} \cdot \frac{d y}{x}\right)\left((S+2 \alpha|Y|)\left(x^{2} \partial_{x}\right)+\hat{Y} \cdot\left(x \partial_{y}\right)\right) \\
=\left(\begin{array}{cc}
S(S+2 \alpha|Y|) & S\langle\hat{Y}, \cdot\rangle \\
\hat{Y}(S+2 \alpha|Y|) & \hat{Y}\langle\hat{Y}, \cdot\rangle
\end{array}\right),
\end{gathered}
$$

with the first column and row corresponding to $\frac{d x}{x^{2}}$, resp. $x^{2} \partial_{x}$, and the second column and row to the (co)normal vectors. For 2-tensors, as before, we use a decomposition

$$
\frac{d x}{x^{2}} \otimes \frac{d x}{x^{2}}, \frac{d x}{x^{2}} \otimes \frac{d y}{x}, \frac{d y}{x} \otimes \frac{d x}{x^{2}}, \frac{d y}{x} \otimes \frac{d y}{x},
$$

where the symmetry of the 2-tensor is the statement that the 2nd and 3rd (block) entries are the same. For the actual endomorphism we write

$$
\begin{aligned}
& \left(\begin{array}{c}
S^{2} \\
S\langle\hat{Y}, \cdot\rangle_{1} \\
S\langle\hat{Y}, \cdot\rangle_{2} \\
\langle\hat{Y}, \cdot\rangle_{1}\langle\hat{Y}, \cdot\rangle_{2}
\end{array}\right)\left((S+2 \alpha|Y|)^{2} \hat{Y}_{1} \hat{Y}_{2} \quad(S+2 \alpha|Y|) \hat{Y}_{1} \hat{Y}_{2}\langle\hat{Y}, \cdot\rangle_{1} \quad(S+2 \alpha|Y|) \hat{Y}_{1} \hat{Y}_{2}\langle\hat{Y}, \cdot\rangle_{2} \quad \hat{Y}_{1} \hat{Y}_{2}\langle\hat{Y}, \cdot\rangle_{1}\langle\hat{Y}, \cdot\rangle_{2}\right) \\
& =\left(\begin{array}{cccc}
S^{2}(S+2 \alpha|Y|)^{2} & S^{2}(S+2 \alpha|Y|)\langle\hat{Y}, \cdot\rangle_{1} & S^{2}(S+2 \alpha|Y|)\langle\hat{Y}, \cdot\rangle_{2} & S^{2}\langle\hat{Y}, \cdot\rangle_{1}\langle\hat{Y}, \cdot\rangle_{2} \\
S(S+2 \alpha|Y|)^{2} Y_{1} & S(S+2 \alpha|Y|) \hat{Y}_{1}\langle\hat{Y}, \cdot\rangle_{1} & S(S+2 \alpha|Y|) \hat{Y}_{1}\langle\hat{Y}, \cdot\rangle_{2} & S \hat{Y}_{1}\langle\hat{Y}, \cdot\rangle_{1}\langle\hat{Y}, \cdot\rangle_{2} \\
S(S+2 \alpha|Y|)^{2} Y_{2} & S(S+2 \alpha|Y|) \hat{Y}_{2}\langle\hat{Y}, .\rangle_{1} & S(S+2 \alpha|Y|) \hat{Y}_{2}\langle\hat{Y}, \cdot\rangle_{2} & S \hat{Y}_{2}\langle\hat{Y}, \cdot\rangle_{1}\langle\hat{Y}, \cdot\rangle_{2} \\
(S+2 \alpha|Y|)^{2} \hat{Y}_{1} \hat{Y}_{2} & (S+2 \alpha|Y|) \hat{Y}_{1} \hat{Y}_{2}\langle\hat{Y}, \cdot\rangle_{1} & (S+2 \alpha|Y|) \hat{Y}_{1} \hat{Y}_{2}\langle\hat{Y}, \cdot\rangle_{2} & \hat{Y}_{1} \hat{Y}_{2}\langle\hat{Y}, \cdot\rangle_{1}\langle\hat{Y}, \cdot\rangle_{2}
\end{array}\right) .
\end{aligned}
$$

Here we write subscripts 1 and 2 for clarity on $\hat{Y}$ to denote whether it is acting on the first or the second factor, though this also immediately follows from its position within the matrix.

Now, the standard principal symbol is that of the conormal singularity at the diagonal, i.e. $X=0, Y=0$. Writing $(X, Y)=Z,(\xi, \eta)=\zeta$, we would need to evaluate the $Z$-Fourier transform as $|\zeta| \rightarrow \infty$. This was discussed in [28] around Equation (3.8); the leading order behavior of the Fourier transform as $|\zeta| \rightarrow \infty$ can be obtained by working on the blown-up space of the diagonal, with coordinates $|Z|, \hat{Z}=\frac{Z}{|Z|}$ (as well as $z=(x, y)$ ), and integrating the restriction of the Schwartz kernel to the front face, $|Z|^{-1}=0$, after removing the singular factor $|Z|^{-n+1}$, along the equatorial sphere corresponding to $\zeta$, and given by $\hat{Z} \cdot \zeta=0$. Now, concretely in our setting, in view of the infinite order vanishing, indeed compact support, of the Schwartz kernel as $X /|Y| \rightarrow \infty$ (and $Y$ bounded), we may work in semi-projective coordinates, i.e. in spherical coordinates in $Y$, but $X /|Y|$ as the normal variable; the equatorial sphere then becomes $(X /|Y|) \xi+\hat{Y} \cdot \eta=0$ (with the integral of course relative to an appropriate positive density). With $\tilde{S}=X /|Y|$, keeping in mind that 
terms with extra vanishing factors at the front face, $|Y|=0$ can be dropped, we thus need to integrate

$$
\left(\begin{array}{cc}
\tilde{S}^{2} & \tilde{S}\langle\hat{Y}, \cdot\rangle \\
\tilde{S} \hat{Y} & \hat{Y}\langle\hat{Y}, \cdot\rangle
\end{array}\right) \chi(\tilde{S})=\left(\begin{array}{c}
\tilde{S} \\
\hat{Y}
\end{array}\right) \otimes\left(\begin{array}{cc}
\tilde{S} & \hat{Y}
\end{array}\right) \chi(\tilde{S})
$$

on this equatorial sphere in the case of one-forms, and the analogous expression in the case of symmetric 2-tensors. Now, for $\chi \geq 0$ this matrix is a positive multiple of the projection to the span of $(\tilde{S}, \hat{Y})$. As $(\tilde{S}, \hat{Y})$ runs through the $(\xi, \eta)$-equatorial sphere, we are taking a positive (in the sense of non-negative) linear combination of the projections to the span of the vectors in this orthocomplement, with the weight being strictly positive as long as $\chi(\tilde{S})>0$ at the point in question. But by Lemma 3.2 , the kernel of the standard principal symbol of $\delta_{\digamma}^{s}$ consists of covectors of the form $v=\left(v_{0}, v^{\prime}\right)$ with $\xi v_{0}+\eta \cdot v^{\prime}=0$. Hence, if we show that for each such non-zero vector $\left(v_{0}, v^{\prime}\right)$ there is at least one $(\tilde{S}, \hat{Y})$ with $\chi(\tilde{S})>0$ and $\xi \tilde{S}+\eta \cdot \hat{Y}=0$ and $\tilde{S} v_{0}+\hat{Y} \cdot v^{\prime} \neq 0$, we conclude that the integral of the projections is positive, thus the principal symbol of our operator is elliptic, on the kernel of the standard principal symbol of $\delta_{\digamma}^{s}$. But this is straightforward if $\chi(0)>0$ :

(1) if $v^{\prime}=0$ then $\xi=0$ (since $v_{0} \neq 0$ ), one may take $\tilde{S} \neq 0$ small, $\hat{Y}$ orthogonal to $\eta$ ( such $\hat{Y}$ exists as $\eta \in \mathbb{R}^{n-1}, n \geq 3$ ),

(2) if $v^{\prime} \neq 0$ and $v^{\prime}$ is not a multiple of $\eta$, then take $\hat{Y}$ orthogonal to $\eta$ but not to $v^{\prime}, \tilde{S}=0$

(3) if $v^{\prime}=c \eta$ with $v^{\prime} \neq 0$ (so $c$ and $\eta$ do not vanish) then $\xi v_{0}+c|\eta|^{2}=0$ so with $\hat{Y}$ still to be chosen if we let $\tilde{S}=-\frac{\eta \cdot \hat{Y}}{\xi}$, then $\tilde{S} v_{0}+\hat{Y} \cdot v^{\prime}=c(\hat{Y} \cdot \eta)\left(1+\frac{|\eta|^{2}}{\xi^{2}}\right)$ which is non-zero as long as $\hat{Y} \cdot \eta \neq 0$; this can be again arranged, together with $\hat{Y} \cdot \eta$ being sufficiently small (such $\hat{Y}$ exists again as $\eta \in \mathbb{R}^{n-1}, n \geq 3$ ), so that $\tilde{S}$ is small enough in order to ensure $\chi(\tilde{S})>0$.

This shows that the principal symbol is positive definite on the kernel of the symbol of $\delta_{\digamma}^{s}$.

In the case of symmetric 2 -tensors, the matrix 3.10 is replaced by

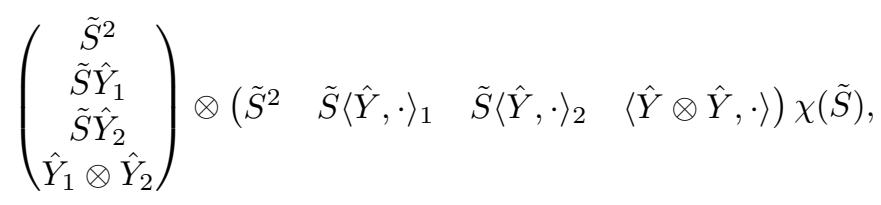

which again is a non-negative multiple of a projection. For a symmetric 2-tensor of the form $v=\left(v_{N N}, v_{N T}, v_{N T}, v_{T T}\right)$ in the kernel of the principal symbol of $\delta_{\digamma}^{s}$, we have by Lemma 3.2 that

$$
\begin{aligned}
& \xi v_{N N}+\eta \cdot v_{N T}=0, \\
& \xi v_{N T}+\frac{1}{2}\left(\eta_{1}+\eta_{2}\right) \cdot v_{T T}=0,
\end{aligned}
$$

where $\eta_{1}$ resp. $\eta_{2}$ denoting that the inner product is taken in the first, resp. second, slots. Taking the inner product of the second equation with $\eta$ gives

$$
\xi \eta \cdot v_{N T}+(\eta \otimes \eta) v_{T T}=0 .
$$

Substituting this into the first equation yields

$$
\xi^{2} v_{N N}=(\eta \otimes \eta) v_{T T}
$$


We now consider two cases, $\xi=0$ and $\xi \neq 0$.

If $\xi \neq 0$, then for a symmetric 2-tensor being in the kernel of the principal symbol of $\delta_{\digamma}^{s}$ at fiber infinity and of (3.11) for $(\tilde{S}, \hat{Y})$ satisfying $\xi \tilde{S}+\eta \cdot \hat{Y}=0$, i.e. $\tilde{S}=-\frac{\eta}{\xi} \cdot \hat{Y}$ is equivalent to

$$
\begin{aligned}
& v_{N N}=\xi^{-2}(\eta \otimes \eta) v_{T T}, \\
& v_{N T}=-\frac{1}{2 \xi}\left(\eta_{1}+\eta_{2}\right) \cdot v_{T T} \\
& \left(\left(\frac{\eta \cdot \hat{Y}}{\xi}\right)^{2} \frac{\eta \otimes \eta}{\xi^{2}}+\frac{\eta \cdot \hat{Y}}{\xi^{2}}\left(\frac{\eta}{\xi} \otimes \hat{Y}+\hat{Y} \otimes \frac{\eta}{\xi}\right)+\hat{Y} \otimes \hat{Y}\right) \cdot v_{T T}=0,
\end{aligned}
$$

and the last equation is equivalent to

$$
\left(\left(\frac{\eta \cdot \hat{Y}}{\xi} \frac{\eta}{\xi}+\hat{Y}\right) \otimes\left(\frac{\eta \cdot \hat{Y}}{\xi} \frac{\eta}{\xi}+\hat{Y}\right)\right) \cdot v_{T T}=0 .
$$

If $\eta=0$, the first two equations say directly that $v_{N N}$ and $v_{N T}$ vanish, while the last one states that $(\hat{Y} \otimes \hat{Y}) \cdot v_{T T}=0$ for all $\hat{Y}$ (we may simply take $\tilde{S}=0$ ); but symmetric 2-tensors of the form $\hat{Y} \otimes \hat{Y}$ span the space of all symmetric 2-tensors (as $\left.w_{1} \otimes w_{2}+w_{2} \otimes w_{1}=\left(w_{1}+w_{2}\right) \otimes\left(w_{1}+w_{2}\right)-w_{1} \otimes w_{1}-w_{2} \otimes w_{2}\right)$, so we conclude that $v_{T T}=0$, and thus $v=0$ in this case. On the other hand, if $\eta \neq 0$ then taking $\hat{Y}=\epsilon \hat{\eta}+\left(1-\epsilon^{2}\right)^{1 / 2} \hat{Y}^{\perp}$ and substituting into this equation yields

$$
\left(\left(1+\frac{|\eta|^{2}}{\xi^{2}}\right)^{2} \epsilon^{2} \hat{\eta} \otimes \hat{\eta}+\left(1+\frac{|\eta|^{2}}{\xi^{2}}\right) \epsilon\left(1-\epsilon^{2}\right)^{1 / 2}\left(\hat{\eta} \otimes \hat{Y}^{\perp}+\hat{Y}^{\perp} \otimes \hat{\eta}\right)+\left(1-\epsilon^{2}\right) \hat{Y}^{\perp} \otimes \hat{Y}^{\perp}\right) \cdot v_{T T}=0 .
$$

Note that $\tilde{S}=-\epsilon \frac{|\eta|}{\xi}$, so $|\tilde{S}|$ is small when $|\epsilon|$ is sufficiently small. Substituting in $\epsilon=0$ yields $\left(\hat{Y}^{\perp} \otimes \hat{Y}^{\perp}\right) \cdot v_{T T}=0$; since cotensors of the form $\hat{Y}^{\perp} \otimes \hat{Y}^{\perp}$ span $\eta^{\perp} \otimes \eta^{\perp}\left(\eta^{\perp}\right.$ being the orthocomplement of $\left.\eta\right)$, we conclude that $v_{T T}$ is orthogonal to every element of $\eta^{\perp} \otimes \eta^{\perp}$. Next, taking the derivative in $\epsilon$ at $\epsilon=0$ yields $\left(\hat{\eta} \otimes \hat{Y}^{\perp}+\hat{Y}^{\perp} \otimes \hat{\eta}\right) \cdot v_{T T}=0$ for all $\hat{Y}^{\perp}$; symmetric tensors of this form, together with $\eta^{\perp} \otimes \eta^{\perp}$, span all tensors in $(\eta \otimes \eta)^{\perp}$. Finally taking the second derivative at $\epsilon=0$ shows that $(\hat{\eta} \otimes \hat{\eta}) \cdot v_{T T}=0$, this in conclusion $v_{T T}=0$. Combined with the first two equations of (3.13), one concludes that $v=0$, thus the desired ellipticity follows.

On the other hand, if $\xi=0$ (and so $\eta \neq 0$ ), then for a symmetric 2-tensor being in the kernel of the principal symbol of $\delta_{\digamma}^{s}$ at fiber infinity and of (3.11) for $(\tilde{S}, \hat{Y})$ satisfying $\xi \tilde{S}+\eta \cdot \hat{Y}=0$, i.e. $\eta \cdot \hat{Y}=0$ is equivalent to

$$
\begin{aligned}
& \eta \cdot v_{N T}=0 \\
& \left(\eta_{1}+\eta_{2}\right) \cdot v_{T T}=0, \\
& \tilde{S}^{2} v_{N N}+2 \tilde{S} \hat{Y} \cdot v_{N T}+(\hat{Y} \otimes \hat{Y}) \cdot v_{T T}=0 .
\end{aligned}
$$

Since there are no constraints on $\tilde{S}$ (apart from $|\tilde{S}|$ small), we can differentiate the last equation up to two times and evaluate the result at 0 to conclude that $v_{N N}=0$, $\hat{Y} \cdot v_{N T}=0$ and $(\hat{Y} \otimes \hat{Y}) \cdot v_{T T}=0$. Combined with the first two equations of (3.14), this shows $v=0$, so again the desired ellipticity follows.

Thus, in summary, both on one forms and on symmetric 2-tensors the principal symbol at fiber infinity is elliptic on the kernel of that of $\delta_{\digamma}^{s}$, proving the lemma. 
Lemma 3.5. For $\digamma>0$ on one forms $N_{\digamma}$ is elliptic at finite points of ${ }^{\mathrm{sc}} T^{*} X$ when restricted to the kernel of the principal symbol of $\delta_{\digamma}^{s}$. On the other hand, there exists $\digamma_{0}>0$ such that on symmetric 2-tensors $N_{\digamma}$ is elliptic at finite points of ${ }^{\mathrm{sc}} T^{*} X$ when restricted to the kernel of the principal symbol of $\delta_{\digamma}^{s}$.

Proof. Again this is similar to, but technically much more involved than, the scalar setting. We recall from 28 , that the kernel is based on using a compactly supported $C^{\infty}$ localizer, $\chi$, but for the actual computation it is convenient to use a Gaussian instead $\chi_{0}$ instead. One recovers the result by taking $\phi \in C_{c}^{\infty}(\mathbb{R}), \phi \geq 0$, identically 1 near 0 , and considering an approximating sequence $\chi_{k}=\phi(. / k) \chi_{0}$. Then the Schwartz kernels at the front face still converge in the space of distributions conormal to the diagonal, which means that the principal symbols (including at finite points) also converge, giving the desired ellipticity for sufficiently large $k$.

Recall that the scattering principal symbol is the Fourier transform of the Schwartz kernel at the front face, so we now need to compute this Fourier transform. We start with the one form case. Taking $\chi(s)=e^{-s^{2} /(2 \nu(\hat{Y}))}$ as in the scalar case considered in [28] for the computation (in the scalar case we took $\nu=\digamma^{-1} \alpha$; here we leave it unspecified for now, except demanding $0<\nu<2 \digamma^{-1} \alpha$ as needed for the Schwartz kernel to be rapidly decreasing at infinity on the front face), we can compute the $X$-Fourier transform exactly as before, keeping in mind that this needs to be evaluated at $-\xi$ (just like the $Y$ Fourier transform needs to be evaluated at $-\eta)$ due to our definition of $X$ :

$$
\begin{aligned}
& |Y|^{2-n} e^{-i \alpha(-\xi-i \digamma)|Y|^{2}}\left(\begin{array}{cc}
D_{\sigma}^{2}-2 \alpha|Y| D_{\sigma} & -D_{\sigma}\langle\hat{Y}, \cdot\rangle \\
\hat{Y}\left(-D_{\sigma}+2 \alpha|Y|\right) & \hat{Y}\langle\hat{Y}, \cdot\rangle
\end{array}\right) \hat{\chi}((-\xi-i \digamma)|Y|) \\
& =c \sqrt{\nu}|Y|^{2-n} e^{i \alpha(\xi+i \digamma)|Y|^{2}}\left(\begin{array}{cc}
D_{\sigma}^{2}-2 \alpha|Y| D_{\sigma} & -D_{\sigma}\langle\hat{Y}, \cdot\rangle \\
\hat{Y}\left(-D_{\sigma}+2 \alpha|Y|\right) & \hat{Y}\langle\hat{Y}, \cdot\rangle
\end{array}\right) e^{-\nu(\xi+i \digamma)^{2}|Y|^{2} / 2}
\end{aligned}
$$

with $c>0$, and with $D_{\sigma}$ differentiating the argument of $\hat{\chi}$. One is left with computing the $Y$-Fourier transform, which in polar coordinates takes the form

$$
\begin{aligned}
\int_{\mathbb{S}^{n-2}} \int_{[0, \infty)} e^{i|Y| \hat{Y} \cdot \eta}|Y|^{2-n} e^{i \alpha(\xi+i \digamma)|Y|^{2}} & \left(\begin{array}{cc}
-D_{\sigma}\left(-D_{\sigma}+2 \alpha|Y|\right) & -D_{\sigma}\langle\hat{Y}, \cdot\rangle \\
\hat{Y}\left(-D_{\sigma}+2 \alpha|Y|\right) & \hat{Y}\langle\hat{Y}, \cdot\rangle
\end{array}\right) \hat{\chi}(-(\xi+i \digamma)|Y|)|Y|^{n-2} d|Y| d \hat{Y},
\end{aligned}
$$

and the factors $|Y|^{ \pm(n-2)}$ cancel as in the scalar case. Explicitly evaluating the derivatives, writing

$$
\phi(\xi, \hat{Y})=\nu(\hat{Y})(\xi+i \digamma)^{2}-2 i \alpha(\hat{Y})(\xi+i \digamma),
$$

yields

$$
\begin{gathered}
\int_{\mathbb{S}^{n-2}} \int_{0}^{\infty} e^{i|Y| \hat{Y} \cdot \eta}\left(\begin{array}{cc}
i \nu(\xi+i \digamma)(i \nu(\xi+i \digamma)+2 \alpha)|Y|^{2}+\nu & i \nu(\xi+i \digamma)|Y|\langle\hat{Y}, \cdot \cdot\rangle \\
\hat{Y}(i \nu(\xi+i \digamma)+2 \alpha)|Y| & \hat{Y}\langle\hat{Y}, \cdot\rangle
\end{array}\right) \\
\times e^{-\phi|Y|^{2} / 2} d|Y| d \hat{Y} .
\end{gathered}
$$

We extend the integral in $|Y|$ to $\mathbb{R}$, replacing it by a variable $t$, and using that the integrand is invariant under the joint change of variables $t \rightarrow-t$ and $\hat{Y} \rightarrow-\hat{Y}$. 
This gives

$$
\begin{aligned}
& \frac{1}{2} \int_{\mathbb{S}^{n-2}} \int_{\mathbb{R}} e^{i t \hat{Y} \cdot \eta} \\
& \left(\begin{array}{cc}
i \nu(\xi+i \digamma)(i \nu(\xi+i \digamma)+2 \alpha) t^{2}+\nu & i \nu(\xi+i \digamma) t\langle\hat{Y}, \cdot\rangle \\
\hat{Y}(i \nu(\xi+i \digamma)+2 \alpha) t & \hat{Y}\langle\hat{Y}, \cdot\rangle
\end{array}\right) \\
& \quad \times e^{-\phi t^{2} / 2} d t d \hat{Y} .
\end{aligned}
$$

Now the $t$ integral is a Fourier transform evaluated at $-\hat{Y} \cdot \eta$, under which multiplication by $t$ becomes $D_{\hat{Y} \cdot \eta}$. Since the Fourier transform of $e^{-\phi(\xi, \hat{Y}) t^{2} / 2}$ is a constant multiple of

$$
\phi(\xi, \hat{Y})^{-1 / 2} e^{-(\hat{Y} \cdot \eta)^{2} /(2 \phi(\xi, \hat{Y}))}
$$

we are left with

$$
\int_{\mathbb{S}^{n-2}} \phi(\xi, \hat{Y})^{-1 / 2}\left(\begin{array}{cc}
i \nu(\xi+i \digamma)(i \nu(\xi+i \digamma)+2 \alpha) D_{\hat{Y} \cdot \eta}^{2}+\nu & i \nu(\xi+i \digamma)\langle\hat{Y}, \cdot\rangle D_{\hat{Y} \cdot \eta} \\
\hat{Y}(i \nu(\xi+i \digamma)+2 \alpha) D_{\hat{Y} \cdot \eta} & \hat{Y}\langle\hat{Y}, \cdot\rangle
\end{array}\right)
$$

which explicitly gives

$$
\begin{gathered}
\int_{\mathbb{S}^{n-2}} \phi(\xi, \hat{Y})^{-1 / 2} \\
\left(\begin{array}{cc}
i \nu(\xi+i \digamma)(i \nu(\xi+i \digamma)+2 \alpha)\left(-\frac{(\hat{Y} \cdot \eta)^{2}}{\phi(\xi, \hat{Y})^{2}}+\frac{1}{\phi(\xi, \hat{Y})}\right)+\nu & i \nu(\xi+i \digamma)\langle\hat{Y}, \cdot\rangle i \frac{\hat{Y} \cdot \eta}{\phi(\xi, \hat{Y})} \\
\hat{Y}(i \nu(\xi+i \digamma)+2 \alpha) i \frac{\hat{Y} \cdot \eta}{\phi(\xi, \hat{Y})} & \hat{Y}\langle\hat{Y}, \cdot\rangle \\
\times e^{-(\hat{Y} \cdot \eta)^{2} /(2 \phi(\xi, \hat{Y}))} d \hat{Y} .
\end{array}\right)
\end{gathered}
$$

Now observe that the top left entry of the matrix is exactly

$$
-\nu \phi(\xi, \hat{Y})\left(-\frac{(\hat{Y} \cdot \eta)^{2}}{\phi(\xi, \hat{Y})^{2}}+\frac{1}{\phi(\xi, \hat{Y})}\right)+\nu=\frac{\nu(\hat{Y} \cdot \eta)^{2}}{\phi(\xi, \hat{Y})}=\nu(\xi+i \digamma)(\nu(\xi+i \digamma)-2 i \alpha) \frac{(\hat{Y} \cdot \eta)^{2}}{\phi(\xi, \hat{Y})^{2}} .
$$

Thus, the matrix in the integrand is

$$
\left(\begin{array}{c}
-\frac{\nu(\xi+i \digamma)}{\phi}(\hat{Y} \cdot \eta) \\
\hat{Y}
\end{array}\right) \otimes\left(-\frac{(\nu(\xi+i \digamma)-2 i \alpha)}{\phi}(\hat{Y} \cdot \eta) \quad\langle\hat{Y}, \cdot\rangle\right) .
$$

Now, if we take

$$
\nu=\digamma^{-1} \alpha
$$

as in the scalar case in $[28$, then

$$
\nu(\xi+i \digamma)-2 i \alpha=\nu(\xi-i \digamma),
$$

while

$$
\phi=(\xi+i \digamma)(\nu(\xi+i \digamma)-2 i \alpha)=\nu\left(\xi^{2}+\digamma^{2}\right)
$$


is real, so the matrix, with this choice of $\nu$, is orthogonal projection to the span of $\left(-\frac{\nu(\xi+i \digamma)}{\phi}(\hat{Y} \cdot \eta), \hat{Y}\right)$. The expression 3.17 becomes

$\left(\xi^{2}+\digamma^{2}\right)^{-1 / 2}$

$$
\int_{\mathbb{S}^{n-2}} \nu^{-1 / 2}\left(\begin{array}{c}
-\frac{\nu(\xi+i \digamma)}{\xi^{2}+\digamma^{2}}(\hat{Y} \cdot \eta) \\
\hat{Y}
\end{array}\right) \otimes\left(-\frac{\nu(\xi-i \digamma)}{\xi^{2}+\digamma^{2}}(\hat{Y} \cdot \eta) \quad\langle\hat{Y}, \cdot\rangle\right) e^{-(\hat{Y} \cdot \eta)^{2} /\left(2 \nu\left(\xi^{2}+\digamma^{2}\right)\right)} d \hat{Y}
$$

which is thus a superposition of positive (in the sense of non-negative) operators, which is thus itself positive. Further, if a vector $\left(v_{0}, v^{\prime}\right)$ lies in the kernel of the principal symbol of $\delta_{\digamma}^{s}$, i.e. $(\xi-i \digamma) v_{0}+\iota_{\eta} v^{\prime}=0$, then orthogonality to $\left(-\frac{\nu(\xi+i \digamma)}{\xi^{2}+\digamma^{2}}(\hat{Y}\right.$. $\eta), \hat{Y}$ ) for any particular $\hat{Y}$ would mean

$$
0=-\frac{\nu(\xi-i \digamma)}{\xi^{2}+\digamma^{2}}(\hat{Y} \cdot \eta) v_{0}+\hat{Y} \cdot v^{\prime}=\frac{\nu}{\xi^{2}+\digamma^{2}}\left(\eta \cdot v^{\prime}\right)(\hat{Y} \cdot \eta)+\hat{Y} \cdot v^{\prime} .
$$

Note that $\mathbb{S}^{n-2}$ is at least one dimensional (i.e. is the sphere in at least a 2dimensional vector space). Consider $v^{\prime} \neq 0$; this would necessarily be the case of interest since $v_{0}=-(\xi-i \digamma)^{-1}\left(\eta \cdot v^{\prime}\right)$. If $\eta=0$, picking $\hat{Y}$ parallel to $v^{\prime}$ shows that there is at least one choice of $\hat{Y}$ for which this equality does not hold. If $\eta \neq 0$, and $v^{\prime}$ is not a multiple of $\eta$, we can take $\hat{Y}$ orthogonal to $\eta$ and not orthogonal to $v^{\prime}$, which again gives a choice of $\hat{Y}$ for the equality above does not hold. Finally, if $v^{\prime}$ is a multiple of $\eta$, the expression at hand is just $\frac{\nu|\eta|^{2}}{\xi^{2}+\digamma^{2}}\left(\hat{Y} \cdot v^{\prime}\right)+\hat{Y} \cdot v^{\prime}$, so choosing any $\hat{Y}$ not orthogonal to $v^{\prime}$ again gives a $\hat{Y}$ for which the equality does not hold. Therefore, (3.18) is actually positive definite when restricted to the kernel of the symbol of $\delta_{\digamma}^{s}$, as claimed.

We now turn to the 2-tensor version. With $B_{i j}$ corresponding to the terms with $i$ factors of $S$ and $j$ factors of $S+2 \alpha|Y|$ prior to the Fourier transform, the analogue of 3.15 is

$$
\begin{aligned}
& \int_{\mathbb{S}^{n-2}} \int_{0}^{\infty} e^{i|Y| \hat{Y} \cdot \eta}\left(\begin{array}{cccc}
B_{22} & B_{21}\langle\hat{Y}, \cdot\rangle_{1} & B_{21}\langle\hat{Y}, \cdot\rangle_{2} & B_{20}\langle\hat{Y}, \cdot\rangle_{1}\langle\hat{Y}, \cdot\rangle_{2} \\
B_{12} Y_{1} & B_{11} \hat{Y}_{1}\langle\hat{Y}, \cdot\rangle_{1} & B_{11} \hat{Y}_{1}\langle\hat{Y}, \cdot\rangle_{2} & B_{10} \hat{Y}_{1}\langle\hat{Y}, \cdot\rangle_{1}\langle\hat{Y}, \cdot\rangle_{2} \\
B_{12} Y_{2} & B_{11} \hat{Y}_{2}\langle\hat{Y}, \cdot\rangle_{1} & B_{11} \hat{Y}_{2}\langle\hat{Y}, \cdot\rangle_{2} & B_{10} \hat{Y}_{2}\langle\hat{Y}, \cdot\rangle_{1}\langle\hat{Y}, \cdot\rangle_{2} \\
B_{02} \hat{Y}_{1} \hat{Y}_{2} & B_{01} \hat{Y}_{1} \hat{Y}_{2}\langle\hat{Y}, \cdot\rangle_{1} & B_{01} \hat{Y}_{1} \hat{Y}_{2}\langle\hat{Y}, \cdot\rangle_{2} & B_{00} \hat{Y}_{1} \hat{Y}_{2}\langle\hat{Y}, \cdot\rangle_{1}\langle\hat{Y}, \cdot\rangle_{2}
\end{array}\right) \\
& \times e^{-\phi|Y|^{2} / 2} d|Y| d \hat{Y},
\end{aligned}
$$

with

$$
\begin{aligned}
& B_{00}=1 \\
& B_{10}=i \nu(\xi+i \digamma)|Y| \\
& B_{20}=-\nu^{2}(\xi+i \digamma)^{2}|Y|^{2}+\nu \\
& B_{01}=i(\nu(\xi+i \digamma)-2 i \alpha)|Y| \\
& B_{11}=-\nu(\xi+i \digamma)(\nu(\xi+i \digamma)-2 i \alpha)|Y|^{2}+\nu \\
& B_{21}=-i \nu^{2}(\xi+i \digamma)^{2}(\nu(\xi+i \digamma)-2 i \alpha)|Y|^{3}+\left(3 i \nu^{2}(\xi+i \digamma)+2 \alpha \nu\right)|Y| \\
& B_{02}=-(\nu(\xi+i \digamma)-2 i \alpha)^{2}|Y|^{2}+\nu \\
& B_{12}=-i \nu(\xi+i \digamma)(\nu(\xi+i \digamma)-2 i \alpha)^{2}|Y|^{3} \\
& B_{22}=\nu^{2}(\xi+i \digamma)^{2}(\nu(\xi+i \digamma)-2 i \alpha)^{2}|Y|^{4}+\nu\left(-6 \nu^{2}(\xi+i \digamma)^{2}+12 i \nu \alpha(\xi+i \digamma)+4 \alpha^{2}\right)|Y|^{2}+3 \nu^{2}
\end{aligned}
$$


Note that the leading term of $B_{j k}$, in terms of the power of $|Y|$ involved, is simply $(i \nu(\xi+i \digamma)|Y|)^{j}(i(\nu(\xi+i \digamma)-2 i \alpha)|Y|)^{k}$; this arises by all derivatives in (3.9) arising by Fourier transforming in $S$ (which gives a derivative $-D_{\sigma}$ in the dual variable $\sigma$ ) falling on the exponential, $e^{-\nu \sigma^{2} / 2}$, which is then evaluated at $\sigma=-(\xi+i \digamma)|Y|$. However, for the full scattering principal symbol all terms are relevant.

Next, we extend the $|Y|$ integral to $\mathbb{R}$, writing the corresponding variable as $t$ and do the Fourier transform in $t$ (with a minus sign, i.e. evaluated at $-\hat{Y} \cdot \eta$ ) as in the one-form setting. This replaces $t$ by $D_{\hat{Y} \cdot \eta}$, as above, and in view of (3.16), explicitly evaluating the derivatives, we obtain the following analogue of (3.17)

$$
\begin{aligned}
& \int_{\mathbb{S}^{n-2}}\left(\begin{array}{cccc}
C_{22} & C_{21}\langle\hat{Y}, \cdot\rangle_{1} & C_{21}\langle\hat{Y}, \cdot\rangle_{2} & C_{20}\langle\hat{Y}, \cdot\rangle_{1}\langle\hat{Y}, \cdot\rangle_{2} \\
C_{12} Y_{1} & C_{11} \hat{Y}_{1}\langle\hat{Y}, .\rangle_{1} & C_{11} \hat{Y}_{1}\langle\hat{Y}, .\rangle_{2} & C_{10} \hat{Y}_{1}\langle\hat{Y}, \cdot\rangle_{1}\langle\hat{Y}, \cdot\rangle_{2} \\
C_{12} Y_{2} & C_{11} \hat{Y}_{2}\langle\hat{Y}, \cdot\rangle_{1} & C_{11} \hat{Y}_{2}\langle\hat{Y}, .\rangle_{2} & C_{10} \hat{Y}_{2}\langle\hat{Y}, \cdot\rangle_{1}\langle\hat{Y}, \cdot\rangle_{2} \\
C_{02} \hat{Y}_{1} \hat{Y}_{2} & C_{01} \hat{Y}_{1} \hat{Y}_{2}\langle\hat{Y}, \cdot\rangle_{1} & C_{01} \hat{Y}_{1} \hat{Y}_{2}\langle\hat{Y}, \cdot\rangle_{2} & C_{00} \hat{Y}_{1} \hat{Y}_{2}\langle\hat{Y}, \cdot\rangle_{1}\langle\hat{Y}, \cdot\rangle_{2}
\end{array}\right) \\
& \times \phi(\xi, \hat{Y})^{-1 / 2} e^{-(\hat{Y} \cdot \eta)^{2} /(2 \phi(\xi, \hat{Y}))} d \hat{Y},
\end{aligned}
$$

where, with $\rho=\hat{Y} \cdot \eta$,

$$
\begin{aligned}
& C_{00}=1, \\
& C_{10}=-\nu(\xi+i \digamma) \phi^{-1} \rho, \\
& C_{20}=\nu^{2}(\xi+i \digamma)^{2} \phi^{-2} \rho^{2}-2 i \alpha \nu \phi^{-1}(\xi+i \digamma), \\
& C_{01}=-(\nu(\xi+i \digamma)-2 i \alpha) \phi^{-1} \rho, \\
& \left.C_{11}=\nu(\xi+i \digamma)\right)(\nu(\xi+i \digamma)-2 i \alpha) \phi^{-2} \rho^{2}, \\
& C_{21}=-\nu^{2}(\xi+i \digamma)^{2}(\nu(\xi+i \digamma)-2 i \alpha) \phi^{-3} \rho^{3}+2 \alpha \nu i \phi^{-1} \rho, \\
& C_{02}=(\nu(\xi+i \digamma)-2 i \alpha)^{2} \phi^{-2} \rho^{2}+\phi^{-1}(\nu(\xi+i \digamma)-2 i \alpha) 2 i \alpha, \\
& C_{12}=-\nu(\xi+i \digamma)(\nu(\xi+i \digamma)-2 i \alpha)^{2} \phi^{-3} \rho^{3}-2 i \alpha \nu \phi^{-1} \rho, \\
& C_{22}=\nu^{2}(\xi+i \digamma)^{2}(\nu(\xi+i \digamma)-2 i \alpha)^{2} \phi^{-4} \rho^{4}-4 \alpha^{2} \nu \phi^{-2} \rho^{2}+4 \alpha^{2} \nu \phi^{-1} .
\end{aligned}
$$

Note again that the highest order term, in terms of the power of $\rho$, of $C_{j k}$ is $(\nu(\xi+i \digamma))^{j}(\nu(\xi+i \digamma)-2 i \alpha)^{k}(-1)^{j+k} \phi^{-j-k}$, corresponding to all derivatives $D_{\rho}$ falling on the exponential $e^{-\rho^{2} /(2 \phi)}$, evaluated at $\rho=\hat{Y} \cdot \eta$.

Notice that $C_{11}$ is exactly the $(1,1)$ entry in the one-form calculation, (3.17), while $C_{10}$, resp. $C_{01}$, are the factors in the $(1,2)$ and $(2,1)$ entries, for similar reasons. Now, it is easy to check that the matrix in $(3.20)$ is

$$
\left(\begin{array}{c}
C_{20} \\
\hat{Y}_{1} C_{10} \\
\hat{Y}_{2} C_{10} \\
\hat{Y}_{1} \hat{Y}_{2}
\end{array}\right) \otimes\left(\begin{array}{llll}
C_{02} & C_{01}\langle\hat{Y}, \cdot\rangle_{1} & C_{01}\langle Y, \cdot\rangle_{2} & \langle\hat{Y}, \cdot\rangle_{1}\langle\hat{Y}, \cdot\rangle_{2}
\end{array}\right) .
$$

Letting $\nu=\digamma^{-1} \alpha$ as in the one-form setting, the second factor here is the adjoint (involving of complex conjugates) of the first, in particular (with $\rho=\hat{Y} \cdot \eta$ )

$C_{01}=-\nu(\xi-i \digamma) \phi^{-1} \rho, C_{02}=\nu^{2}(\xi-i \digamma)^{2} \phi^{-2} \rho^{2}+2 i \alpha \nu(\xi-i \digamma) \phi^{-1}, \phi=\nu\left(\xi^{2}+\digamma^{2}\right)$,

so $(3.21)$ is just a positive multiple of projection to the span of $\left(C_{20}, \hat{Y}_{1} C_{10}, \hat{Y}_{2} C_{10}, \hat{Y}_{1} \hat{Y}_{2}\right)$. Thus, as in the one form setting, we have a superposition of positive (in the sense 
of non-negative) operators, so it remains to check that as $\hat{Y}$ varies, these vectors span the kernel of $\delta_{\digamma}^{s}$.

For a symmetric 2-tensor of the form $v=\left(v_{N N}, v_{N T}, v_{N T}, v_{T T}\right)$ in the kernel of the principal symbol of $\delta_{\digamma}^{s}$, we have by Lemma 3.2 that

$$
\begin{aligned}
& (\xi-i \digamma) v_{N N}+\eta \cdot v_{N T}+a \cdot v_{T T}=0, \\
& (\xi-i \digamma) v_{N T}+\frac{1}{2}\left(\eta_{1}+\eta_{2}\right) \cdot v_{T T}=0,
\end{aligned}
$$

where $\eta_{1}$ resp. $\eta_{2}$ denoting that the inner product is taken in the first, resp. second, slots. Taking the inner product of the second equation with $\eta$ gives

$$
(\xi-i \digamma) \eta \cdot v_{N T}+(\eta \otimes \eta) \cdot v_{T T}=0
$$

Substituting this into the first equation yields

$$
(\xi-i \digamma)^{2} v_{N N}+((\xi-i \digamma) a-\eta \otimes \eta) \cdot v_{T T}=0,
$$

SO

$v_{N N}=(\xi-i \digamma)^{-2}(\eta \otimes \eta-(\xi-i \digamma) a) \cdot v_{T T}, v_{N T}=-2^{-1}(\xi-i \digamma)^{-1}\left(\eta_{1}+\eta_{2}\right) \cdot v_{T T}$.

For a fixed $\hat{Y}$ for $v$ in the kernel of the symbol of $\delta_{\digamma}^{s}$ to be in the kernel of the projection 3.21 means that

$\left(C_{02}(\xi-i \digamma)^{-2}(\eta \otimes \eta-(\xi-i \digamma) a)-C_{01}(\xi-i \digamma)^{-1}(\eta \otimes \hat{Y}+\hat{Y} \otimes \eta)+\hat{Y} \otimes \hat{Y}\right) \cdot v_{T T}=0$,

so recalling $\nu=\digamma^{-1} \alpha, \phi=\nu\left(\xi^{2}+\digamma^{2}\right)$,

$$
\begin{gathered}
\left((\xi+i \digamma)^{-1}\left(\xi^{2}+\digamma^{2}\right)^{-1}(\hat{Y} \cdot \eta)^{2}+2 i \alpha\left(\xi^{2}+\digamma^{2}\right)^{-1}\right)\left((\xi-i \digamma)^{-1}(\eta \otimes \eta)-a\right) \\
\left.+\left(\xi^{2}+\digamma^{2}\right)^{-1}(\hat{Y} \cdot \eta)(\eta \otimes \hat{Y}+\hat{Y} \otimes \eta)+\hat{Y} \otimes \hat{Y}\right) \cdot v_{T T}=0 .
\end{gathered}
$$

Now, it is convenient to rewrite this in terms of 'semiclassical' (in $h=\digamma^{-1}$ ) variables

It becomes

$$
\xi_{\digamma}=\xi / \digamma, \eta_{\digamma}=\eta / \digamma \text {. }
$$

$$
\begin{gathered}
\left(\left(\xi_{\digamma}+i\right)^{-1}\left(\xi_{\digamma}^{2}+1\right)^{-1}\left(\hat{Y} \cdot \eta_{\digamma}\right)^{2}+2 i \digamma^{-1} \alpha\left(\xi_{\digamma}^{2}+1\right)^{-1}\right)\left(\left(\xi_{\digamma}-i\right)^{-1}\left(\eta_{\digamma} \otimes \eta_{\digamma}\right)-\digamma^{-1} a\right) \\
\left.+\left(\xi_{\digamma}^{2}+1\right)^{-1}\left(\hat{Y} \cdot \eta_{\digamma}\right)\left(\eta_{\digamma} \otimes \hat{Y}+\hat{Y} \otimes \eta_{\digamma}\right)+\hat{Y} \otimes \hat{Y}\right) \cdot v_{T T}=0 .
\end{gathered}
$$

Letting $\digamma^{-1}=h \rightarrow 0$, one obtains

$$
\begin{aligned}
\left(\left(\xi_{\digamma}\right.\right. & +i)^{-1}\left(\xi_{\digamma}^{2}+1\right)^{-1}\left(\hat{Y} \cdot \eta_{\digamma}\right)^{2}\left(\xi_{\digamma}-i\right)^{-1}\left(\eta_{\digamma} \otimes \eta_{\digamma}\right) \\
& \left.+\left(\xi_{\digamma}^{2}+1\right)^{-1}\left(\hat{Y} \cdot \eta_{\digamma}\right)\left(\eta_{\digamma} \otimes \hat{Y}+\hat{Y} \otimes \eta_{\digamma}\right)+\hat{Y} \otimes \hat{Y}\right) \cdot v_{T T}=0,
\end{aligned}
$$

i.e.

$$
\left(\left(\left(\xi_{\digamma}^{2}+1\right)^{-1}\left(\hat{Y} \cdot \eta_{\digamma}\right) \eta_{\digamma}+\hat{Y}\right) \otimes\left(\left(\xi_{\digamma}^{2}+1\right)^{-1}\left(\hat{Y} \cdot \eta_{\digamma}\right) \eta_{\digamma}+\hat{Y}\right)\right) \cdot v_{T T}=0 .
$$

One can see that this last equation, when it holds for all $\hat{Y}$, implies the vanishing of $v_{T T}$ just as for the principal symbol at fiber infinity. Indeed, if $\eta_{\digamma}=0$ then we have $(\hat{Y} \otimes \hat{Y}) \cdot v_{T T}=0$ for all $\hat{Y}$, and symmetric 2-tensors of the form $\hat{Y} \otimes \hat{Y} \operatorname{span}$ the space of all symmetric 2-tensors (as $w_{1} \otimes w_{2}+w_{2} \otimes w_{1}=\left(w_{1}+w_{2}\right) \otimes\left(w_{1}+\right.$ $\left.\left.w_{2}\right)-w_{1} \otimes w_{1}-w_{2} \otimes w_{2}\right)$, so we conclude that $v_{T T}=0$, and thus $v=0$ in this case. 
On the other hand, if $\eta_{\digamma} \neq 0$ then taking $\hat{Y}=\epsilon \hat{\eta}_{\digamma}+\left(1-\epsilon^{2}\right)^{1 / 2} \hat{Y}^{\perp}$ and substituting into this equation yields

$$
\begin{aligned}
((1+ & \left.\frac{\left|\eta_{\digamma}\right|^{2}}{\xi_{\digamma}^{2}+1}\right)^{2} \epsilon^{2} \hat{\eta}_{\digamma} \otimes \hat{\eta}_{\digamma}+\left(1+\frac{\left|\eta_{\digamma}\right|^{2}}{\xi_{\digamma}^{2}+1}\right) \epsilon\left(1-\epsilon^{2}\right)^{1 / 2}\left(\hat{\eta}_{\digamma} \otimes \hat{Y}^{\perp}+\hat{Y}^{\perp} \otimes \hat{\eta}_{\digamma}\right) \\
& \left.+\left(1-\epsilon^{2}\right) \hat{Y}^{\perp} \otimes \hat{Y}^{\perp}\right) \cdot v_{T T}=0 .
\end{aligned}
$$

Substituting in $\epsilon=0$ yields $\left(\hat{Y}^{\perp} \otimes \hat{Y}^{\perp}\right) \cdot v_{T T}=0$; since cotensors of the form $\hat{Y}^{\perp} \otimes \hat{Y}^{\perp} \operatorname{span} \eta_{\digamma}^{\perp} \otimes \eta_{\digamma}^{\perp}\left(\eta_{\digamma}^{\perp}\right.$ being the orthocomplement of $\left.\eta_{\digamma}\right)$, we conclude that $v_{T T}$ is orthogonal to every element of $\eta_{\digamma}^{\perp} \otimes \eta_{\digamma}^{\perp}$. Next, taking the derivative in $\epsilon$ at $\epsilon=0$ yields $\left(\hat{\eta}_{\digamma} \otimes \hat{Y}^{\perp}+\hat{Y}^{\perp} \otimes \hat{\eta}_{\digamma}\right) \cdot v_{T T}=0$ for all $\hat{Y}^{\perp}$; symmetric tensors of this form, together with $\eta_{\digamma}^{\perp} \otimes \eta_{\digamma}^{\perp}$, span all tensors in $\left(\eta_{\digamma} \otimes \eta_{\digamma}\right)^{\perp}$. Finally taking the second derivative at $\epsilon=0$ shows that $\left(\hat{\eta}_{\digamma} \otimes \hat{\eta}_{\digamma}\right) \cdot v_{T T}=0$, this in conclusion $v_{T T}=0$. Combined with the first two equations of (3.13), one concludes that $v=0$. Correspondingly one concludes that for sufficiently large $\digamma>0$ one has ellipticity at all finite points, which proves the lemma.

As already explained, this lemma completes the proof of Proposition 3.3 .

\section{The gauge CONDition And the Proof of the MAIN RESUlts}

The still remaining analytic issue is to check that we can arrange the gauge condition, $\delta_{\digamma}^{s} f_{\digamma}=0$. We do this by considering various regions $\Omega_{j}$, which are manifolds with corners: they have the artificial boundary, $\partial X$, which is 'at infinity' in the scattering calculus sense, as well as the 'interior' boundary $\partial_{\text {int }} \Omega_{j}$, which could be $\partial M$, or another (farther away) hypersurface.

Recall that our gauge freedom is that we can add to $f$ (without changing $I f$ ) any tensor of the form $d^{s} v$, with $v$ vanishing at $\partial M$ or on a hypersurface further away, such as $\partial_{\text {int }} \Omega_{j}$, i.e. to $f_{\digamma}=e^{-\digamma / x} f$ (without changing $I e^{\digamma / x} f_{\digamma}$ ) any tensor of the form $d_{\digamma}^{s} v_{\digamma}=e^{-\digamma / x} d e^{\digamma / x} v_{\digamma}$ with a similar vanishing condition. If we let $\Delta_{\digamma, s}=\delta_{\digamma}^{s} d_{\digamma}^{s}$ be the 'solenoidal Witten Laplacian', and we impose Dirichlet boundary condition on $\partial_{\text {int }} \Omega_{j}$ (to get the desired vanishing for $v_{\digamma}$ ), and we show that $\Delta_{\digamma, s}$ is invertible (with this boundary condition) on suitable function spaces, then

$$
\begin{aligned}
& \mathcal{S}_{\digamma, \Omega_{j}} \phi=\phi_{\digamma, \Omega_{j}}^{s}=\phi-d_{\digamma}^{s} \Delta_{\digamma, s, \Omega_{j}}^{-1} \delta_{\digamma}^{s} \phi, \\
& \mathcal{P}_{\digamma, \Omega_{j}} \phi=d_{\digamma}^{s} Q_{\digamma, \Omega_{j}} \phi, \quad Q_{\digamma, \Omega_{j}} \phi=\Delta_{\digamma, s, \Omega_{j}}^{-1} \delta_{\digamma}^{s} \phi,
\end{aligned}
$$

are the solenoidal $(\mathcal{S})$, resp. potential $(\mathcal{P})$ projections of $\phi$ on $\Omega_{j}$. Notice that $\mathcal{P}_{\digamma, \Omega_{j}} \phi$ is indeed in the range of $d_{\digamma}^{s}$ applied to a function or one-form vanishing at $\partial_{\mathrm{int}} \Omega_{j}$ thanks to the boundary condition for $\Delta_{\digamma, s}$, which means that $Q_{\digamma, \Omega_{j}}$ maps to such functions or tensors. Thus $\mathcal{S}_{\digamma, \Omega_{j}} \phi$ differs from $\phi$ by such a tensor, so $I e^{\digamma / x} f_{\digamma}=I e^{\digamma / x} \mathcal{S}_{\digamma, \Omega_{j}} f_{\digamma}$. Further,

$$
\delta^{s} \mathcal{S}_{\digamma, \Omega_{j}} \phi=\delta_{\digamma}^{s} \phi-\delta_{\digamma}^{s} d_{\digamma}^{s} \Delta_{\digamma, s, \Omega_{j}}^{-1} \delta_{\digamma}^{s} \phi=0
$$

so $\delta_{\digamma}^{s} f_{\digamma}=0$, i.e. the gauge condition we want to impose is in fact satisfied.

Thus, it remains to check the invertibility of $\Delta_{\digamma, s}$ with the desired boundary condition. Before doing this we remark:

Lemma 4.1. For $\digamma>0$, the operator $\Delta_{\digamma, s}=\delta_{\digamma}^{s} d_{\digamma}^{s}$ is (jointly) elliptic in $\operatorname{Diff}_{\mathrm{sc}}^{2,0}(X)$ on functions. 
On the other hand, there exists $\digamma_{0}>0$ such that for $\digamma \geq \digamma_{0}$ the operator $\Delta_{\digamma, s}=\delta_{\digamma}^{s} d_{\digamma}^{s}$ is (jointly) elliptic in $\operatorname{Diff}_{\mathrm{sc}}^{2,0}\left(X ;{ }^{\mathrm{sc}} T^{*} X,{ }^{\mathrm{sc}} T^{*} X\right)$ on one forms. In fact, on one forms (for all $\digamma>0$ )

$$
\delta_{\digamma}^{s} d_{\digamma}^{s}=\frac{1}{2} \nabla_{\digamma}^{*} \nabla_{\digamma}+\frac{1}{2} d_{\digamma} \delta_{\digamma}+A+R,
$$

where $R \in x \operatorname{Diff}_{\mathrm{sc}}^{1}\left(X ;{ }^{\mathrm{sc}} T^{*} X,{ }^{\mathrm{sc}} T^{*} X\right), A \in \operatorname{Diff}_{\mathrm{sc}}^{1}\left(X ;{ }^{\mathrm{sc}} T^{*} X ;{ }^{\mathrm{sc}} T^{*} X\right)$ is independent of $\digamma$ and where $\nabla^{\digamma}=e^{-\digamma / x} \nabla e^{\digamma / x}$, with $\nabla$ gradient relative to $g_{\mathrm{sc}}$ (not $g$ ), $d_{\digamma}=$ $e^{-\digamma / x} d e^{\digamma / x}$ the exterior derivative on functions, while $\delta_{\digamma}$ is its adjoint on oneforms.

Proof. Most of the computations for this lemma have been performed in Lemma 3.2 In particular, the symbolic computation is algebraic, and can be done pointwise, where one arranges that $g_{\mathrm{sc}}$ is as in Lemma 3.2. Since the function case is simpler, we consider one-forms. Thus the full principal symbol of $d_{\digamma}^{s}$ (with symmetric 2tensors considered as a subspace of 2 -tensors) is

$$
\left(\begin{array}{cc}
\xi+i \digamma & 0 \\
\frac{1}{2} \eta \otimes & \frac{1}{2}(\xi+i \digamma) \\
\frac{1}{2} \eta \otimes & \frac{1}{2}(\xi+i \digamma) \\
a & \eta \otimes_{s}
\end{array}\right),
$$

that of $\delta_{\digamma}^{s}$ is

$$
\left(\begin{array}{cccc}
\xi-i \digamma & \frac{1}{2} \iota_{\eta} & \frac{1}{2} \iota_{\eta} & \langle a, .\rangle \\
0 & \frac{1}{2}(\xi-i \digamma) & \frac{1}{2}(\xi-i \digamma) & \iota_{\eta}^{s}
\end{array}\right) .
$$

with the lower right block having $(\ell i j)$ entry given by $\frac{1}{2}\left(\eta_{i} \delta_{\ell j}+\eta_{j} \delta_{i \ell}\right)$. Correspondingly, the product, $\Delta_{\digamma}^{s}$, has symbol

$$
\left(\begin{array}{cc}
\xi^{2}+\digamma^{2}+\frac{1}{2}|\eta|^{2} & \frac{1}{2}(\xi+i \digamma) \iota_{\eta} \\
\frac{1}{2}(\xi-i \digamma) \eta \otimes & \frac{1}{2}\left(\xi^{2}+\digamma^{2}\right)+\iota_{\eta}^{s} \eta \otimes_{s}
\end{array}\right)+\left(\begin{array}{cc}
\langle a, .\rangle a & \langle a, .\rangle \eta \otimes_{s} \\
\iota_{\eta}^{s} a & 0
\end{array}\right)
$$

with the lower right block having $\ell k$ entry $\frac{1}{2}\left(\xi^{2}+\digamma^{2}\right) \delta_{\ell k}+\frac{1}{2}|\eta|^{2} \delta_{\ell k}+\frac{1}{2} \eta_{\ell} \eta_{k}$, and where we separated out the $a$ terms.

Now ellipticity is easy to see if $a=0$, with a $\digamma$-dependent lower bound then, and this can be used to absorb the $a$ term by taking $\digamma>0$ sufficiently large.

To make this more explicit, however, we note that, similarly, the principal symbol of the gradient relative to $g_{\mathrm{sc}}$ is

$$
\left(\begin{array}{cc}
\xi & 0 \\
\eta \otimes & 0 \\
0 & \xi \\
0 & \eta \otimes
\end{array}\right)
$$

with no non-zero entry in the lower left hand corner unlike for the $g$-gradient in [3.8), and thus the adjoint $\nabla_{\digamma}^{*}$ of $\nabla_{\digamma}$ has principal symbol

$$
\left(\begin{array}{cccc}
\xi-i \digamma & \iota_{\eta} & 0 & 0 \\
0 & 0 & \xi-i \digamma & \iota_{\eta}^{s}
\end{array}\right)
$$

Correspondingly, $\nabla_{\digamma}^{*} \nabla_{\digamma}$ has symbol

$$
\left(\begin{array}{cc}
\xi^{2}+\digamma^{2}+|\eta|^{2} & 0 \\
0 & \xi^{2}+\digamma^{2}+|\eta|^{2}
\end{array}\right)
$$


which is certainly elliptic (including at finite points in ${ }^{\mathrm{sc}} T_{\partial X}^{*} X$ !), and indeed is simply $\xi^{2}+\digamma^{2}+|\eta|^{2}$ times the identity matrix. Now, $d=d^{s}$ going from functions to one-forms has symbol $\left(\begin{array}{l}\xi \\ \eta\end{array}\right)$, so its conjugate $e^{-\digamma / x} d e^{\digamma / x}$ has symbol $\left(\begin{array}{c}\xi+i \digamma \\ \eta\end{array}\right)$, its adjoint, $\delta_{\digamma}$ has symbol $\left(\begin{array}{lll}\xi-i \digamma & \iota_{\eta}\end{array}\right)$, and now $d_{\digamma} \delta_{\digamma}$ has symbol

$$
\left(\begin{array}{cc}
\xi^{2}+\digamma^{2} & (\xi+i \digamma) \iota_{\eta} \\
(\xi-i \digamma) \eta & \eta \otimes \iota_{\eta}
\end{array}\right)
$$

Combining these, we see that the first term in 4.2 , i.e. in the principal symbol of $\delta_{\digamma}^{s} d^{s} \digamma$, is the same as $\frac{1}{2} \nabla_{\digamma}^{*} \nabla_{\digamma}+\frac{1}{2} d_{\digamma} \delta_{\digamma}$, with both terms non-negative, and the first actually positive definite, with a lower bound $\xi^{2}+\digamma^{2}+|\eta|^{2}$ times the identity. This proves 4.1), with the principal symbol of $A$ given by the second term in 4.2), which is in particular independent of $\digamma$. Since with $C$ a bound for $a$, the symbol of $A$ is bounded by $C^{2}+2 C|\eta| \leq C^{2}\left(1+\epsilon^{-1}\right)+\epsilon|\eta|^{2}$ for any $\epsilon>0$, in particular $\epsilon<1$, this shows that the principal symbol of $\delta_{\digamma}^{s} d^{s} \digamma$ is positive definite if $\digamma>0$ is chosen large enough, completing the proof of the lemma.

We now turn to the invertibility question. Let $\dot{H}_{\mathrm{sc}}^{m, l}\left(\Omega_{j}\right)$ be the subspace of $H_{\mathrm{sc}}^{m, l}(X)$ consisting of distributions supported in $\overline{\Omega_{j}}$, and let $\bar{H}_{\mathrm{sc}}^{m, l}\left(\Omega_{j}\right)$ the space of restrictions of elements of $H_{\mathrm{sc}}^{m, l}(X)$ to $\Omega_{j}$. Thus, $\dot{H}_{\mathrm{sc}}^{m, l}\left(\Omega_{j}\right)^{*}=\bar{H}_{\mathrm{sc}}^{-m,-l}\left(\Omega_{j}\right)$. Here we shall be mostly interested in $m=1, l=0$; then at $\partial_{\text {int }} \Omega_{j}$, away from $\partial X$, $\dot{H}_{\mathrm{sc}}^{1,0}\left(\Omega_{j}\right)$ is the standard $H_{0}^{1}$-space (which is $\dot{H}^{1}$ in Hörmander's notation, which we adopt), while $\bar{H}_{\mathrm{sc}}^{-1,0}$ is $H^{-1}$ there (which is $\bar{H}^{-1}$ in Hörmander's notation). Further, $\dot{C}^{\infty}\left(\Omega_{j}\right)$, with the dot denoting infinite order vanishing at all boundary hypersurfaces, or indeed $C_{c}^{\infty}\left(\Omega_{j}\right)$ (compact support), are dense in $H_{\mathrm{sc}}^{1,0}$, so $\dot{H}_{\mathrm{sc}}^{1,0}\left(\Omega_{j}\right)$ is the completion of these spaces in the $H_{\mathrm{sc}}^{1,0}(X)$-norm. In addition, the norm on $H_{\mathrm{sc}}^{1,0}(X)$ is equivalent to $\|\nabla u\|_{L^{2}}^{2}+\|u\|_{L^{2}}^{2}$, where the norms are with respect to any scattering metric, and $\nabla$ is any differential operator with principal symbol given by $d$, such as the gradient relative to any (perhaps different from the one giving the norm) scattering metric. For $L_{\mathrm{sc}}^{2}=H_{\mathrm{sc}}^{0,0}$, or for the weighted $L^{2}$-spaces $H_{\mathrm{sc}}^{0, l}$, the dots and bars do not make any difference (do not change the space) as usual. Further, the inclusion map $\dot{H}_{\mathrm{sc}}^{1,1} \rightarrow L^{2}$ (or indeed even $\bar{H}_{\mathrm{sc}}^{1,1} \rightarrow L^{2}$ ) is compact. As usual, all these spaces can be defined for sections of vector bundles, such as ${ }^{\mathrm{sc}} T_{\Omega_{j}}^{*} X$, by local trivializations. The norm on $\dot{H}_{\mathrm{sc}}^{1,0}\left(\Omega_{j},{ }^{\mathrm{sc}} T^{*} \Omega_{j}\right)$ is still induced by a gradient $\nabla$ with respect to any scattering differential operator the same way.

Lemma 4.2. The operator on functions $\Delta_{\digamma, s}=\delta_{\digamma}^{s} d_{\digamma}^{s}$, considered as a map $\dot{H}_{\mathrm{sc}}^{1,0} \rightarrow$ $\left(\dot{H}_{\mathrm{sc}}^{1,0}\right)^{*}=\bar{H}_{\mathrm{sc}}^{-1,0}$ is invertible for all $\digamma>0$.

On the other hand, there exists $\digamma_{0}>0$ such that for $\digamma \geq \digamma_{0}$, the operator $\Delta_{\digamma, s}=\delta_{\digamma}^{s} d_{\digamma}^{s}$ on one forms is invertible.

Remark 4.3. The reason for having some $\digamma_{0}>0$, and requiring $\digamma \geq \digamma_{0}$, in the one form case (rather than merely $\digamma>0$ ) is that $d^{s}$ is relative to a standard metric $g$, not a scattering metric. The proof given below in fact shows that if $d^{s}$ is replaced by $d_{g_{\mathrm{sc}}}^{s}$, relative to any scattering metric $g_{\mathrm{sc}}$, then one may simply assume $\digamma>0$.

Proof. The following considerations apply to both the function case and the oneform case. Relative to the scattering metric with respect to which $\delta^{s}$ is defined, the quadratic form of $\Delta_{\digamma, s}$ is $\left\langle\Delta_{\digamma, s} u, v\right\rangle=\left\langle d_{\digamma}^{s} u, d_{\digamma}^{s} v\right\rangle$. So in particular

$$
\left\|d_{\digamma}^{s} u\right\|_{L^{2}}^{2} \leq\left\|\Delta_{\digamma, s} u\right\|_{\bar{H}_{\mathrm{sc}}^{-1,0}}\|u\|_{\dot{H}_{\mathrm{sc}}^{1,0}} \leq \epsilon^{-1}\left\|\Delta_{\digamma, s} u\right\|_{\bar{H}_{\mathrm{sc}}^{-1,0}}^{2}+\epsilon\|u\|_{\dot{H}_{\mathrm{sc}}^{1,0}}^{2} .
$$


Correspondingly, if one has an estimate

$$
\|u\|_{\dot{H}_{\mathrm{sc}}^{1,0}} \leq C\left\|d_{\digamma}^{s} u\right\|_{L^{2}}
$$

or equivalently (for a different $C$ )

$$
\|\nabla u\|_{L^{2}}+\|u\|_{L^{2}} \leq C\left\|d_{\digamma}^{s} u\right\|_{L^{2}}
$$

then for small $\epsilon>0$, one can absorb $\epsilon\|u\|_{\dot{H}_{\mathrm{sc}}^{1,0}}^{2}$ into the left hand side above, giving

$$
\|u\|_{\dot{H}_{\mathrm{sc}}^{1,0}} \leq C\left\|d_{\digamma}^{s} u\right\|_{L^{2}} \leq C^{\prime}\left\|\Delta_{\digamma, s} u\right\|_{\bar{H}_{\mathrm{sc}}^{-1,0}} .
$$

This in turn gives invertibility in the sense discussed in the statement of the theorem since $\Delta_{\digamma, s}$ is formally (and as this shows, actually) self-adjoint, so one has the same estimates for the formal adjoint.

On the other hand, if one has an estimate

$$
\|u\|_{\dot{H}_{\mathrm{sc}}^{1,0}} \leq C\left\|d_{\digamma}^{s} u\right\|_{L^{2}}+C\|u\|_{\dot{H}_{\mathrm{sc}}^{0,-1}}
$$

or equivalently

$$
\|\nabla u\|_{L^{2}}+\|u\|_{L^{2}} \leq C\left\|d_{\digamma}^{s} u\right\|_{L^{2}}+C\|u\|_{\dot{H}_{\mathrm{sc}}^{0,-1}}
$$

then for $\epsilon>0$ small one gets

$$
\|u\|_{\dot{H}_{\mathrm{sc}}^{1,0}} \leq C\left\|d_{\digamma}^{s} u\right\|_{L^{2}}+C\|u\|_{\dot{H}_{\mathrm{sc}}^{0,1}} \leq C^{\prime}\left\|\Delta_{\digamma, s} u\right\|_{\bar{H}_{\mathrm{sc}}^{-1,0}}+C^{\prime}\|u\|_{\dot{H}_{\mathrm{sc}}^{0,-1}}
$$

Again, by formal self-adjointness, one gets the same statement for the adjoint, which implies that $\Delta_{\digamma, s}$ is Fredholm (by virtue of the compactness of the inclusion $\dot{H}_{\text {sc }}^{1,0} \rightarrow \dot{H}_{\text {sc }}^{0,-1}$ ), and further that the invertibility is equivalent to the lack of kernel on $\dot{H}_{\mathrm{sc}}^{1,0}$ (since the cokernel statement follows by formal self-adjointness). Note that 4.5 follows quite easily from Lemma 4.1 (and is standard on functions as $d^{s}=\nabla$ then), in the form case using the Dirichlet boundary condition to apply 4.1) to $u$ and pair with $u$ but we discuss invertibility, taking advantage of Lemma 4.1 later.

Now, on functions, $d^{s}=\nabla$, and as $d_{\digamma}^{s}$ differs from $d^{s}$ by a 0th order operator, $\|\nabla u\|_{L^{2}} \leq C\left\|d_{\digamma}^{s} u\right\|_{L^{2}}+C\|u\|_{L^{2}}$ automatically. In particular, 4.4 follows if one shows $\|u\|_{L^{2}} \leq C\left\|d_{\digamma}^{s} u\right\|_{L^{2}}$ for $u \in \dot{H}_{\mathrm{sc}}^{1,0}$, or equivalently (by density) for $u \in C_{c}^{\infty}\left(\Omega_{j}\right)$, which is a Poincaré inequality.

To prove this Poincaré inequality, notice that $\left\|e^{-\digamma / x}\left(x^{2} D_{x}\right) e^{\digamma / x} u\right\|_{L^{2}} \leq C\left\|d_{\digamma}^{s} u\right\|_{L^{2}}$ certainly, so it suffices to estimate $\|u\|_{L^{2}}$ in terms of the $L^{2}$ norm of

$$
e^{-\digamma / x}\left(x^{2} D_{x}\right) e^{\digamma / x} u=\left(x^{2} D_{x}+i \digamma\right) u .
$$

But for any operator $P$, writing $P_{R}=\left(P+P^{*}\right) / 2$ and $P_{I}=\left(P-P^{*}\right) /(2 i)$ for the symmetric and skew-symmetric parts,

$$
\|P u\|^{2}=\left\|P_{R} u\right\|^{2}+\left\|P_{I} u\right\|^{2}+\left\langle i\left[P_{R}, P_{I}\right] u, u\right\rangle .
$$

It is convenient here to use a metric $\frac{d x^{2}}{x^{4}}+\frac{h}{x^{2}}$ where $h$ is a metric, independent of $x$, on the level sets of $x$, using some product decomposition. For then the metric density is $x^{-(n+1)}|d x||d h|$, so with $P=x^{2} D_{x}+i \digamma, P^{*}=x^{2} D_{x}+i(n-1) x-i \digamma$, so

$$
P_{R}=x^{2} D_{x}+i \frac{n-1}{2} x, P_{I}=\digamma-\frac{n-1}{2} x, i\left[P_{R}, P_{I}\right]=i x^{2} \frac{n-1}{2},
$$


we have

$$
\begin{aligned}
\left\|\left(x^{2} D_{x}+i \digamma\right) u\right\|_{L^{2}}^{2}= & \left\|\left(x^{2} D_{x}+i \frac{n-1}{2}\right) u\right\|_{L^{2}}^{2} \\
+ & \left\|\left(\digamma-\frac{n-1}{2} x\right) u\right\|_{L^{2}}^{2}-\frac{n-1}{2}\left\langle x^{2} u, u\right\rangle \\
= & \left\|\left(x^{2} D_{x}+i \frac{n-1}{2}\right) u\right\|_{L^{2}}^{2} \\
& +\left\langle\left(\left(\digamma-\frac{n-1}{2} x\right)^{2}-\frac{n-1}{2} x^{2}\right) u, u\right\rangle .
\end{aligned}
$$

Now, if $\Omega_{j} \subset\left\{x \leq x_{0}\right\}$, as long as $x_{0}>0$ is sufficiently small so that

$$
\left(\digamma-\frac{n-1}{2} x\right)^{2}-\frac{n-1}{2} x^{2}
$$

is positive (and thus bounded below by a positive constant) on $\left[0, x_{0}\right]$, which is automatic for sufficiently small $x_{0}$, or indeed for bounded $x_{0}$ and sufficiently large $\digamma$, one obtains that $\|u\|_{L^{2}}^{2} \leq C\left\|\left(x^{2} D_{x}+i \digamma\right) u\right\|_{L^{2}}^{2}$, and thus in summary that

$$
\|u\|_{L^{2}} \leq C\left\|d_{\digamma}^{s} u\right\|_{L^{2}}
$$

as desired. This proves the lemma for functions, at least in the case of sufficiently small $x_{0}$.

This actually suffices for our application, but in fact one can do better by noting that in fact even in general this gives us the estimate

$$
\|u\|_{L^{2}} \leq C\left\|d_{\digamma}^{s} u\right\|+C\|u\|_{L^{2}\left(\left\{x_{1} \leq x \leq x_{0}\right\}\right)}
$$

for suitable small $x_{1}>0$. But by the standard Poincaré inequality, using the vanishing at $x=x_{0}$, one can estimate the last term in terms of $C^{\prime}\left\|d_{\digamma}^{s} u\right\|$, which gives the general conclusion for functions. Here, to place us properly in the standard Poincare setting, we note that with $\phi=e^{\digamma / x} u$, the last required estimate is equivalent to the weighted estimate $\left\|e^{-\digamma / x} \phi\right\|_{L^{2}\left(\left\{x_{1} \leq x \leq x_{0}\right\}\right)} \leq C\left\|e^{-\digamma / x} d \phi\right\|_{L^{2}\left(\left\{x_{1} \leq x \leq x_{0}\right\}\right)}$, and now the weights are bounded, so can be dropped completely.

It remains to deal with one-forms. For this we use that 4.1 and 4.3 give that

$$
\delta_{\digamma}^{s} d_{\digamma}^{s}=\frac{1}{2} \nabla^{*} \nabla+\frac{1}{2} \digamma^{2}+\frac{1}{2} d_{\digamma} \delta_{\digamma}+A+\tilde{R},
$$

where $A \in \operatorname{Diff}_{\mathrm{sc}}^{1}(X)$ is independent of $\digamma$ and $\tilde{R} \in x \operatorname{Diff}_{\mathrm{sc}}^{1}(X)$; this follows by rewriting $\nabla_{\digamma}^{*} \nabla_{\digamma}$ using (4.3), which modifies $R$ in 4.1) to give 4.6. Thus, in fact

$$
\left\|d_{\digamma}^{s} u\right\|^{2}=\frac{1}{2}\|\nabla u\|^{2}+\frac{1}{2} \digamma^{2}\|u\|^{2}+\frac{1}{2}\left\|\delta_{\digamma}^{s} u\right\|^{2}+\langle A u, u\rangle+\langle\tilde{R} u, u\rangle .
$$

Since $A \in \operatorname{Diff}_{\mathrm{sc}}^{1}(X),|\langle A u, u\rangle| \leq C\|u\|_{\dot{H}_{\mathrm{sc}}^{1,0}}\|u\|_{L^{2}}$, and there is a similar estimate for the last term. This gives an estimate, for sufficiently large $\digamma$,

$$
\|\nabla u\|^{2}+\digamma^{2}\|u\|^{2} \leq C\left\|d_{\digamma}^{s} u\right\|^{2}+C\left\|x^{1 / 2} u\right\|^{2},
$$

with the constant $C$ on the right hand side depending on $\digamma$, and thus

$$
\langle(1-C x) u, u\rangle \leq C\left\|d_{\digamma}^{s} u\right\|^{2} .
$$

Again, if $x_{0}$ is sufficiently small, this gives

$$
\|u\| \leq C\left\|d_{\digamma}^{s} u\right\|,
$$

and thus the invertibility, while if $x_{0}$ is larger, this still gives

$$
\|u\|_{L^{2}} \leq C\left\|d_{\digamma}^{s} u\right\|_{L^{2}}+C\|u\|_{L^{2}\left(\left\{x_{1} \leq x \leq x_{0}\right\}\right)} .
$$


One can then finish the proof as above, using the standard Poincaré inequality for one forms, see [23, Section 6, Equation (28)].

A slight modification of the argument gives:

Lemma 4.4. The operator on functions $\Delta_{\digamma, s}=\delta_{\digamma}^{s} d_{\digamma}^{s}$, considered as a map $\dot{H}_{\mathrm{sc}}^{1, r} \rightarrow$ $\bar{H}_{\mathrm{sc}}^{-1, r}$ is invertible for all $\digamma>0$ and all $r \in \mathbb{R}$.

On the other hand, there exists $\digamma_{0}>0$ such that for $\digamma \geq \digamma_{0}$, the operator $\Delta_{\digamma, s}=\delta_{\digamma}^{s} d_{\digamma}^{s}$ on one forms is invertible as a map $\dot{H}_{\mathrm{sc}}^{1, r} \rightarrow \bar{H}_{\mathrm{sc}}^{-1, r}$ for all $r \in \mathbb{R}$.

Proof. Since the function case is completely analogous, we consider one forms to be definite. Also note that (full) elliptic regularity would automatically give this result if not for $\partial_{\text {int }} \Omega_{j}$.

An isomorphism estimate $\Delta_{\digamma, s}: \dot{H}_{\mathrm{sc}}^{1, r} \rightarrow \bar{H}_{\mathrm{sc}}^{-1, r}$ is equivalent to an isomorphism estimate $x^{-r} \Delta_{\digamma, s} x^{r}: \dot{H}_{\mathrm{sc}}^{1,0} \rightarrow \bar{H}_{\mathrm{sc}}^{-1,0}$. But the operator on the left is $\Delta_{\digamma, s}+F$, where $F \in x \operatorname{Diff}_{\mathrm{sc}}^{1}$. Thus, $x^{-r} \Delta_{\digamma, s} x^{r}$ is of the form 4.6, with only $\tilde{R}$ changed. The rest of the proof then immediately goes through.

Before proceeding with the analysis of the Dirichlet Laplacian, we first discuss the analogue of Korn's inequality that will be useful later.

Lemma 4.5. Suppose $\Omega_{j}$ is a domain in $X$ as above. For $\digamma>0$ and $r \in \mathbb{R}$,

$$
\|u\|_{\bar{H}_{\mathrm{sc}}^{1, r}\left(\Omega_{j}\right)} \leq C\left(\left\|x^{-r} d_{\digamma}^{s} u\right\|_{L_{\mathrm{sc}}^{2}\left(\Omega_{j}\right)}+\|u\|_{x^{-r} L_{\mathrm{sc}}^{2}\left(\Omega_{j}\right)}\right) .
$$

for one-forms $u \in \bar{H}_{\mathrm{sc}}^{1, r}\left(\Omega_{j}\right)$.

Proof. First note that if one lets $\tilde{u}=x^{-r} u$, then $\|u\|_{\bar{H}_{\mathrm{sc}}^{1, r}\left(\Omega_{j}\right)}$ is equivalent to $\|\tilde{u}\|_{\bar{H}_{\mathrm{sc}}^{1,0}\left(\Omega_{j}\right)}$, and $\left\|x^{-r} d_{\digamma}^{s} u\right\|_{L_{\mathrm{sc}}^{2}\left(\Omega_{j}\right)}+\|u\|_{x^{-r} L_{\mathrm{sc}}^{2}\left(\Omega_{j}\right)}$ is equivalent to $\left\|d_{\digamma}^{s} \tilde{u}\right\|_{L_{\mathrm{sc}}^{2}\left(\Omega_{j}\right)}+$ $\|\tilde{u}\|_{L_{\mathrm{sc}}^{2}\left(\Omega_{j}\right)}$ since the commutator term through $d_{\digamma}^{s}$ can be absorbed into a sufficiently large multiple of $\|u\|_{x^{-r} L_{\mathrm{sc}}^{2}\left(\Omega_{j}\right)}=\|\tilde{u}\|_{L_{\mathrm{sc}}^{2}\left(\Omega_{j}\right)}$. Thus, one is reduced to proving the case $r=0$.

Let $\tilde{\Omega}_{j}$ be a domain in $X$ with $C^{\infty}$ boundary, transversal to $\partial X$, containing $\overline{\Omega_{j}}$. We claim that there is a continuous extension map $E: \bar{H}_{\mathrm{sc}}^{1,0}\left(\Omega_{j}\right) \rightarrow \dot{H}_{\mathrm{sc}}^{1,0}\left(\tilde{\Omega}_{j}\right)$ such that

$$
\left\|d_{\digamma}^{s} E u\right\|_{L_{\mathrm{sc}}^{2}\left(\tilde{\Omega}_{j}\right)}+\|E u\|_{L_{\mathrm{sc}}^{2}\left(\tilde{\Omega}_{j}\right)} \leq C\left(\left\|d_{\digamma}^{s} u\right\|_{L_{\mathrm{sc}}^{2}\left(\Omega_{j}\right)}+\|u\|_{L_{\mathrm{sc}}^{2}\left(\Omega_{j}\right)}\right), \quad u \in \bar{H}_{\mathrm{sc}}^{1,0}\left(\Omega_{j}\right),
$$

i.e. $E u$ is also continuous when on both sides the gradient is replaced by the symmetric gradient in the definition of an $H^{1}$-type space. Once this is proved, the lemma can be shown in the following manner. By 4.1 of Lemma 4.1 any $v \in \dot{H}_{\mathrm{sc}}^{1,0}\left(\tilde{\Omega}_{j}\right)$, in particular $v=E u$, satisfies, for any $\epsilon>0$,

$$
\begin{aligned}
\|\nabla v\|_{L_{\mathrm{sc}}^{2}\left(\tilde{\Omega}_{j}\right)}^{2}+\|v\|_{L_{\mathrm{sc}}^{2}\left(\tilde{\Omega}_{j}\right)}^{2} & \leq 2\left\|d_{\digamma}^{s} v\right\|_{L_{\mathrm{sc}}^{2}\left(\tilde{\Omega}_{j}\right)}^{2}+\|v\|_{L_{\mathrm{sc}}^{2}\left(\tilde{\Omega}_{j}\right)}^{2}+C\|v\|_{L_{\mathrm{sc}}^{2}\left(\tilde{\Omega}_{j}\right)}\|v\|_{\bar{H}_{\mathrm{sc}}^{1,0}\left(\tilde{\Omega}_{j}\right)} \\
& \leq 2\left\|d_{\digamma}^{s} v\right\|_{L_{\mathrm{sc}}^{2}\left(\tilde{\Omega}_{j}\right)}^{2}+C^{\prime}\|v\|_{L_{\mathrm{sc}}^{2}\left(\tilde{\Omega}_{j}\right)}^{2}+\epsilon\|v\|_{\bar{H}_{\mathrm{sc}}^{1,0}\left(\tilde{\Omega}_{j}\right)}^{2}
\end{aligned}
$$

and now for $\epsilon>0$ small, the last term on the right hand side can be absorbed into the left hand side. Using this with $v=E u$, noting that $E$ is an extension map so

$$
\|\nabla u\|_{L_{\mathrm{sc}}^{2}\left(\Omega_{j}\right)}^{2}+\|u\|_{L_{\mathrm{sc}}^{2}\left(\Omega_{j}\right)}^{2} \leq\|\nabla E u\|_{L_{\mathrm{sc}}^{2}\left(\tilde{\Omega}_{j}\right)}^{2}+\|E u\|_{L_{\mathrm{sc}}^{2}\left(\tilde{\Omega}_{j}\right)}^{2},
$$

we deduce, using 4.9 in the last step, that

$$
\|u\|_{\bar{H}_{\mathrm{sc}}^{1,0}\left(\Omega_{j}\right)} \leq C\left(\left\|d_{\digamma}^{s} E u\right\|_{L_{\mathrm{sc}}^{2}\left(\tilde{\Omega}_{j}\right)}+\|E u\|_{L_{\mathrm{sc}}^{2}\left(\tilde{\Omega}_{j}\right)}\right) \leq C^{\prime}\left(\left\|d_{\digamma}^{s} u\right\|_{L_{\mathrm{sc}}^{2}\left(\Omega_{j}\right)}+\|u\|_{L_{\mathrm{sc}}^{2}\left(\Omega_{j}\right)}\right),
$$


completing the proof of the lemma.

Thus, it remains to construct $E$. By a partition of unity, this can be reduced to a local extension, local on $X$. Since $\partial \Omega_{j}$ is transversal to $\partial X$, near points on $\partial X \cap \partial \Omega_{j}$ one can arrange that locally (in a model in which a neighborhood of $p$ is identified with an open set in $\left.\overline{\mathbb{R}^{n}}\right) \partial \Omega_{j}$ is the hypersurface $x_{n}=0, \Omega_{j}$ is $x_{n}>0$; the analogous arrangement can also be made away from $\partial X$ near points on $\partial \Omega_{j}$. Since $H_{\mathrm{sc}}^{s, r}(X), \dot{H}_{\mathrm{sc}}^{s, r}\left(\tilde{\Omega}_{j}\right), \bar{H}_{\mathrm{sc}}^{s, r}\left(\Omega_{j}\right)$, are locally, and also for compactly supported elements in the chart, are preserved by local diffeomorphisms of $X$ to $\overline{\mathbb{R}^{n}}$ in the sense that $X$ is replaced by $\overline{\mathbb{R}^{n}}, \overline{\Omega_{j}}$ by $\overline{\mathbb{R}_{+}^{n}}$ (by virtue of these spaces are well defined on manifolds with boundary, without additional information on metrics, etc., up to equivalence of norms), it suffices to prove that there is a local extension map $E_{1}$ that has the desired properties.

Let $\Phi_{k}\left(x^{\prime}, x_{n}\right)=\left(x^{\prime},-k x_{n}\right)$ for $x_{n}<0$, and consider a variation of the standard construction of an $H^{1}\left(\mathbb{R}_{+}^{n}\right)$ extension map on one-forms as follows. (Note that the usual extension map is given by trivialization of a bundle, in this case using $d x_{j}$ as a local basis of sections, and extending the coefficients using the extension map on functions.) Let $E_{1}$ given by

$$
\left(E_{1} \sum_{j} u_{j} d x_{j}\right)\left(x^{\prime}, x_{n}\right)=\sum_{k=1}^{3} c_{k} \Phi_{k}^{*}\left(\sum u_{j} d x_{j}\right), x_{n}<0,
$$

and

$$
\left(E_{1} \sum_{j} u_{j} d x_{j}\right)\left(x^{\prime}, x_{n}\right)=\sum u_{j} d x_{j}, x_{n} \geq 0
$$

with $c_{k}$ chosen so that $E_{1}: C^{1}\left(\overline{\mathbb{R}_{+}^{n}}\right) \rightarrow C^{1}\left(\mathbb{R}^{n}\right)$. We can achieve this mapping property as follows. We have, with $\partial_{j}$ acting as derivatives on the components, or equivalently but invariantly as Lie derivatives in this case,

$$
\begin{aligned}
\Phi_{k}^{*} u_{j} d x_{j} & =u_{j}\left(x^{\prime},-k x_{n}\right) d x_{j}, j \neq n, \\
\Phi_{k}^{*} u_{n} d x_{n} & =-k u_{n}\left(x^{\prime},-k x_{n}\right) d x_{n}, \\
\partial_{i} \Phi_{k}^{*} u_{j} d x_{j} & =\left(\partial_{i} u_{j}\right)\left(x^{\prime},-k x_{n}\right) d x_{j}, i, j \neq n, \\
\partial_{i} \Phi_{k}^{*} u_{n} d x_{n} & =-k\left(\partial_{i} u_{n}\right)\left(x^{\prime},-k x_{n}\right) d x_{n}, i \neq n, \\
\partial_{n} \Phi_{k}^{*} u_{j} d x_{j} & =-k\left(\partial_{n} u_{j}\right)\left(x^{\prime},-k x_{n}\right) d x_{j}, j \neq n, \\
\partial_{n} \Phi_{k}^{*} u_{n} d x_{n} & =k^{2}\left(\partial_{n} u_{j}\right)\left(x^{\prime},-k x_{n}\right) d x_{n},
\end{aligned}
$$

so the requirements for matching the derivatives at $x_{n}=0$, which gives the $C^{1}$ property, are, for $j \neq n$,

$$
\begin{array}{r}
c_{1}+c_{2}+c_{3}=1, \\
-c_{1}-2 c_{2}-3 c_{3}=1,
\end{array}
$$

while for $j=n$

$$
\begin{aligned}
-c_{1}-2 c_{2}-3 c_{3} & =1, \\
c_{1}+4 c_{2}+9 c_{3} & =1,
\end{aligned}
$$

which gives a 3 -by-3 system

$$
\left(\begin{array}{ccc}
1 & 1 & 1 \\
-1 & -2 & -3 \\
1 & 4 & 9
\end{array}\right)\left(\begin{array}{l}
c_{1} \\
c_{2} \\
c_{3}
\end{array}\right)=\left(\begin{array}{l}
1 \\
1 \\
1
\end{array}\right)
$$


The matrix on the right is a Vandermonde matrix, and is thus invertible, so one can find $c_{k}$ with the desired properties. With this, $E_{1}: C_{c}^{1}\left(\overline{\mathbb{R}_{+}^{n}}\right) \rightarrow C_{c}^{1}\left(\mathbb{R}^{n}\right)$ has the property that $\left\|E_{1} u\right\|_{H^{1}\left(\mathbb{R}^{n}\right)} \leq C\|u\|_{H^{1}\left(\mathbb{R}_{+}^{n}\right)}$, since each term in the definition of $E_{1}$ has derivatives $\partial_{i}$ satisfying $\left\|\partial_{i} \Phi_{k}^{*} u\right\|_{L^{2}\left(\mathbb{R}^{n}\right)} \leq C\left\|\partial_{i} u\right\|_{L^{2}\left(\mathbb{R}_{+}^{n}\right)}$, and since $E_{1} u \in$ $C_{c}^{1}\left(\mathbb{R}^{n}\right)$ assures that the distributional derivative satisfies $\partial_{i} E_{1} u \in L^{2}\left(\mathbb{R}^{n}\right)$, whose square norm can be calculated as the sum of the squared norms over $\mathbb{R}_{+}^{n}=\left\{x_{n}>0\right\}$ and $\mathbb{R}_{-}^{n}=\left\{x_{n}<0\right\}$. Correspondingly, $E_{1}$ extends continuously, in a unique manner, to a map $H^{1}\left(\mathbb{R}_{+}^{n}\right) \rightarrow H^{1}\left(\mathbb{R}^{n}\right)$.

Before proceeding we note that with this choice of coefficients, $E_{1}$ defined as the analogous map on functions, is actually the standard $H^{2}$ extension map. However, on one-forms the same choice, defined in terms of pull-backs, i.e. natural operations, as above, rather than trivializing the form bundle, does not extend continuously to $H^{2}$. On the other hand, if one trivializes the bundle and uses the $H^{2}$ extension map, one does not have the desired property (4.9) for symmetric differentials, a property that we check below with our choice of extension map.

Notice that, with $\Phi_{k}^{*}$ acting on 2-tensors as usual, for all $i, j$,

$$
d x_{i} \otimes\left(\partial_{i} \Phi_{k}^{*} u_{j} d x_{j}\right)+\left(\partial_{j} \Phi_{k}^{*} u_{i} d x_{i}\right) \otimes d x_{j}=\Phi_{k}^{*}\left(\left(\partial_{i} u_{j}+\partial_{j} u_{i}\right) d x_{i} \otimes d x_{j}\right),
$$

as follows from a direct calculation, or indeed from the naturality of the symmetric gradient $d^{s}=d_{g_{0}}^{s}$ for a translation invariant Riemannian metric $g_{0}$ : the two sides are the $i j$ component of $2 d^{s} \Phi_{k}^{*}$, resp. $2 \Phi_{k}^{*} d^{s}$, as for such a metric the symmetric gradient is actually independent of the choice of the metric (in this class). Since, summed over $i, j$, the left hand side is the symmetric gradient of $\Phi_{k}^{*} \sum u_{j} d x_{j}$ in $x_{n}<0$, while the right hand side is the pull-back of the symmetric gradient from $x_{n}>0$, this shows that

$$
\left\|d^{s} \Phi_{k}^{*} u\right\|_{L^{2}\left(\mathbb{R}_{-}^{n}\right)} \leq C\left\|d^{s} u\right\|_{L^{2}\left(\mathbb{R}_{+}^{n}\right)} .
$$

This proves that one has

$$
\left\|d^{s} E_{1} u\right\|_{L^{2}\left(\mathbb{R}^{n}\right)} \leq C\left\|d^{s} u\right\|_{L^{2}\left(\mathbb{R}_{+}^{n}\right)} .
$$

Now, using a partition of unity $\left\{\rho_{k}\right\}$ to localize on $\Omega_{j}$, as mentioned above, this gives a global extension map from $H^{1}\left(\Omega_{j}\right): \sum \psi_{k} E_{1, k} \rho_{k}$, where $\psi_{k}$ is identically 1 near supp $\rho_{k}$. While $d^{s}$ depends on the choice of a metric, the dependence is via the 0 th order term, i.e. one has $d_{g}^{s} u=d_{g_{0}}^{s} u+R u$ for an appropriate 0th order $R$. Using the Euclidean metric in the local model, this shows that

$$
\left\|d_{g}^{s} \psi_{k} E_{1, k} \rho_{k} u\right\|_{L^{2}\left(\mathbb{R}^{n}\right)} \leq C\left(\left\|d_{g_{0}}^{s} \rho_{k} u\right\|_{L^{2}\left(\mathbb{R}_{+}^{n}\right)}+\left\|\rho_{k} u\right\|_{L^{2}\left(\mathbb{R}_{+}^{n}\right)}\right) .
$$

Since $d_{\digamma}^{s}$ differs from $d^{s}$ by a 0 th order term, one can absorb this in the $L^{2}$ norm (using also the continuity of the extension map from $L^{2}$ to $L^{2}$ ):

$$
\left\|d_{g, \digamma}^{s} \psi_{k} E_{1, k} \rho_{k} u\right\|_{L^{2}\left(\mathbb{R}^{n}\right)} \leq C\left(\left\|d_{g_{0}, \digamma}^{s} \rho_{k} u\right\|_{L^{2}\left(\mathbb{R}_{+}^{n}\right)}+\left\|\rho_{k} u\right\|_{L^{2}\left(\mathbb{R}_{+}^{n}\right)}\right) .
$$

Summing over $k$ proves 4.9 , and thus the lemma.

We now return to the analysis of the Dirichlet Laplacian.

Corollary 4.6. Let $\phi \in C_{c}^{\infty}\left(\overline{\Omega_{j}} \backslash \partial_{\mathrm{int}} \Omega_{j}\right)$. Then on functions, for $\digamma>0, k \in \mathbb{R}$, the operator $\phi \Delta_{\digamma, s}^{-1} \phi: \bar{H}_{\mathrm{sc}}^{-1, k} \rightarrow \dot{H}_{\mathrm{sc}}^{1, k}$ is in $\Psi_{\mathrm{sc}}^{-2,0}(X)$. There is $\digamma_{0}>0$ such that the analogous conclusion holds for one forms for $\digamma \geq \digamma_{0}$. 
Proof. This follows from the usual parametrix identity. Namely, by Lemma 4.1. $\Delta_{\digamma, s}$ has a parametrix $B \in \Psi_{\mathrm{sc}}^{-2,0}(X)$ so that

$$
B \Delta_{\digamma, s}=\operatorname{Id}+F_{L}, \Delta_{\digamma, s} B=\operatorname{Id}+F_{R},
$$

with $F_{L}, F_{R} \in \Psi_{\mathrm{sc}}^{-\infty,-\infty}(X)$. Let $\psi \in C_{c}^{\infty}\left(\overline{\Omega_{j}} \backslash \partial_{\text {int }} \Omega_{j}\right)$ be identically 1 on $\operatorname{supp} \phi$. Thus,

$$
\psi=B \Delta_{\digamma, s} \psi-F_{L} \psi=B \psi \Delta_{\digamma, s}+B\left[\Delta_{\digamma, s}, \psi\right]-F_{L} \psi
$$

and

$$
\psi=\psi \Delta_{\digamma, s} B-\psi F_{R}=\Delta_{\digamma, s} \psi B+\left[\psi, \Delta_{\digamma, s}\right] B-\psi F_{R}
$$

Then

$$
\begin{aligned}
\psi \Delta_{\digamma, s}^{-1} \psi= & \psi \Delta_{\digamma, s}^{-1} \Delta_{\digamma, s} \psi B+\psi \Delta_{\digamma, s}^{-1}\left(\left[\psi, \Delta_{\digamma, s}\right] B-\psi F_{R}\right) \\
=\psi^{2} B+ & B \psi \Delta_{\digamma, s} \Delta_{\digamma, s}^{-1}\left(\left[\psi, \Delta_{\digamma, s}\right] B-\psi F_{R}\right) \\
& +\left(B\left[\Delta_{\digamma, s}, \psi\right]-F_{L} \psi\right) \Delta_{\digamma, s}^{-1}\left(\left[\psi, \Delta_{\digamma, s}\right] B-\psi F_{R}\right) \\
=\psi^{2} B+ & B \psi\left(\left[\psi, \Delta_{\digamma, s}\right] B-\psi F_{R}\right) \\
& +\left(B\left[\Delta_{\digamma, s}, \psi\right]-F_{L} \psi\right) \Delta_{\digamma, s}^{-1}\left(\left[\psi, \Delta_{\digamma, s}\right] B-\psi F_{R}\right) .
\end{aligned}
$$

Multiplying from both the left and the right by $\phi$ gives

$$
\begin{aligned}
\phi \Delta_{\digamma, s}^{-1} \phi=\phi B \phi+ & \phi B \psi\left(\left[\psi, \Delta_{\digamma, s}\right] B-\psi F_{R}\right) \phi \\
& +\phi\left(B\left[\Delta_{\digamma, s}, \psi\right]-F_{L} \psi\right) \Delta_{\digamma, s}^{-1}\left(\left[\psi, \Delta_{\digamma, s}\right] B-\psi F_{R}\right) \phi .
\end{aligned}
$$

Now, the first two terms on the right hand side are in $\Psi_{\mathrm{sc}}^{-2,0}$, resp. $\Psi_{\mathrm{sc}}^{-\infty,-\infty}$, in the latter case using the disjointness of $\operatorname{supp} d \psi$ and $\phi$ for $\left[\psi, \Delta_{\digamma, s}\right] B \phi$, resp. that $F_{L} \in \Psi_{\mathrm{sc}}^{-\infty,-\infty}$ for $\psi F_{R} \phi$. For this reason, $\left(\left[\psi, \Delta_{\digamma, s}\right] B-\psi F_{R}\right) \phi$ and $\phi\left(B\left[\Delta_{\digamma, s}, \psi\right]-\right.$ $\left.F_{L} \psi\right)$ are smoothing, in the sense that they map $H_{\mathrm{sc}}^{s, r}(X)$ to $H_{\mathrm{sc}}^{s^{\prime}, r^{\prime}}(X)$ for any $s^{\prime}, r^{\prime}, s, r$, and they also have support so that they map into functions supported in $\overline{\Omega_{j}} \backslash \partial_{\text {int }} \Omega_{j}$, and they also can be applied to functions on $\overline{\Omega_{j}}$. As $\Delta_{\digamma, s}^{-1}$ is continuous $\bar{H}_{\mathrm{sc}}^{-1, k}\left(\Omega_{j}\right) \rightarrow \dot{H}_{\mathrm{sc}}^{1, k}\left(\Omega_{j}\right)$, this shows that the last term is continuous from $H_{\mathrm{sc}}^{s, r}(X)$ to $H_{\mathrm{sc}}^{s^{\prime}, r^{\prime}}(X)$ for any $s^{\prime}, r^{\prime}, s, r$, which means that it has a Schwartz (rapidly decaying with all derivatives) Schwartz kernel, i.e. it is in $\Psi_{\mathrm{sc}}^{-\infty,-\infty}(X)$. This completes the proof.

Corollary 4.7. Let $\phi \in C_{c}^{\infty}\left(\overline{\Omega_{j}} \backslash \partial_{\mathrm{int}} \Omega_{j}\right), \chi \in C^{\infty}\left(\overline{\Omega_{j}}\right)$ with disjoint support and with $\chi$ constant near $\partial_{\mathrm{int}} \Omega_{j}$. Let $\digamma, \digamma_{0}$ as in Corollary 4.6. Then the operator $\chi \Delta_{\digamma, s}^{-1} \phi: \bar{H}_{\mathrm{sc}}^{-1, k}\left(\Omega_{j}\right) \rightarrow \dot{H}_{\mathrm{sc}}^{1, k}\left(\Omega_{j}\right)$ in fact maps $H_{\mathrm{sc}}^{s, r}(X) \rightarrow \dot{H}_{\mathrm{sc}}^{1, k}\left(\Omega_{j}\right)$ for all $s, r, k$.

Similarly, $\phi \Delta_{\digamma, s}^{-1} \chi: \bar{H}_{\mathrm{sc}}^{-1, k}\left(\Omega_{j}\right) \rightarrow \dot{H}_{\mathrm{sc}}^{1, k}\left(\Omega_{j}\right)$ in fact maps $\bar{H}_{\mathrm{sc}}^{-1, k}\left(\Omega_{j}\right) \rightarrow H_{\mathrm{sc}}^{s, r}(X)$ for all $s, r, k$.

Proof. Since the second statement follows by duality, it suffices to prove the first.

As $\chi \phi=0$, we can write

$$
\chi \Delta_{\digamma, s}^{-1} \phi=\left[\chi, \Delta_{\digamma, s}^{-1}\right] \phi=\Delta_{\digamma, s}^{-1}\left[\Delta_{\digamma, s}, \chi\right] \Delta_{\digamma, s}^{-1} \phi .
$$

By Corollary 4.6. $\left[\Delta_{\digamma, s}, \chi\right] \Delta_{\digamma, s}^{-1} \phi \in \Psi_{\mathrm{sc}}^{-\infty, \infty}(X)$ since it is in $\Psi_{\mathrm{sc}}^{-1,0}(X)$ (this uses $\operatorname{supp} d \chi$ disjoint from $\partial_{\mathrm{int}} \Omega_{j}$ ) but $d \chi$ and $\phi$ have disjoint supports. Thus, it maps $H_{\mathrm{sc}}^{s, r}(X) \rightarrow H_{\mathrm{sc}}^{-1, k}(X)$, and thus, in view of $\operatorname{supp} d \chi$, to $\bar{H}_{\mathrm{sc}}^{-1, k}\left(\Omega_{j}\right)$, giving the conclusion. 
Corollary 4.8. Let $\phi \in C_{c}^{\infty}\left(\overline{\Omega_{j}} \backslash \partial_{\text {int }} \Omega_{j}\right), \chi \in C^{\infty}\left(\overline{\Omega_{j}}\right)$ with disjoint support and with $\chi$ constant near $\partial_{\text {int }} \Omega_{j}$. Let $\digamma, \digamma_{0}$ as in Corollary 4.6 .

Then $\phi \mathcal{S}_{\digamma, \Omega_{j}} \phi \in \Psi_{\mathrm{sc}}^{0,0}(X)$, while $\chi \mathcal{S}_{\digamma, \Omega_{j}} \phi: H_{\mathrm{sc}}^{s, r}(X) \rightarrow x^{k} L_{\mathrm{sc}}^{2}\left(\Omega_{j}\right)$ and $\phi \mathcal{S}_{\digamma, \Omega_{j}} \chi:$ $x^{k} L_{\mathrm{sc}}^{2}\left(\Omega_{j}\right) \rightarrow H_{\mathrm{sc}}^{s, r}(X)$ for all $s, r, k$.

Proof. This is immediate from $\mathcal{S}_{\digamma, \Omega_{j}}=\mathrm{Id}-d_{\digamma}^{s} \Delta_{\digamma, s, \Omega_{j}}^{-1} \delta_{\digamma}^{s}$ and the above results concerning $\Delta_{\digamma, s, \Omega_{j}}^{-1}$, using that $d_{\digamma}^{s}$ and $\delta_{\digamma}^{s}$ are differential operators, and thus preserve supports.

We also need the Poisson operator associated to $\partial_{\text {int }} \Omega_{j}$. First note that if $H$ is a (codimension 1) hypersurface in $\overline{\Omega_{j}}$ which intersects $\partial \Omega_{j}$ away from $\partial_{\mathrm{int}} \Omega_{j}$, and does so transversally, then the restriction map

$$
\gamma_{H}: \dot{C}^{\infty}\left(\overline{\Omega_{j}}\right) \rightarrow \dot{C}^{\infty}(H),
$$

with the dots denoting infinite order vanishing at $\partial \Omega_{j}$, resp. $\partial H$, as usual, in fact maps, for $s>1 / 2$,

$$
\gamma_{H}: H_{\mathrm{sc}}^{s, r}\left(\Omega_{j}\right) \rightarrow H_{\mathrm{sc}}^{s-1 / 2, r}(H)
$$

continuously. This can be easily seen since the restriction map is local, and locally in $\overline{\Omega_{j}}$, one can map a neighborhood of $p \in \partial H$ to a neighborhood of a point $p^{\prime} \in \partial \mathbb{R}^{n-1}$ in $\overline{\mathbb{R}^{n}}$ by a diffeomorphism so that $H$ is mapped to $\overline{\mathbb{R}^{n-1}}$, and thus by the diffeomorphism invariance of the spaces under discussion, the standard $\mathbb{R}^{n}$ result with the usual Sobolev spaces $H^{s}\left(\mathbb{R}^{n}\right)=H_{\mathrm{sc}}^{s, 0}\left(\overline{\mathbb{R}^{n}}\right)$, using that weights commute with the restriction, gives 4.10. The same argument also shows that there is a continuous extension map

$$
e_{H}: H_{\mathrm{sc}}^{s-1 / 2, r}(H) \rightarrow H_{\mathrm{sc}}^{s, r}\left(\Omega_{j}\right), \quad \gamma_{H} e_{H}=\mathrm{Id},
$$

since the analogous result on $\mathbb{R}^{n}$ is standard, and one can localize by multiplying by cutoffs without destroying the desired properties.

Considering $\overline{\Omega_{j}}$ inside a larger domain $\overline{\Omega^{\prime}}$, with $\partial_{\text {int }} \Omega_{j}$ satisfying the assumptions for $H$, we have a continuous extension map $\bar{H}_{\mathrm{sc}}^{s, r}\left(\Omega_{j}\right) \rightarrow \bar{H}_{\mathrm{sc}}^{s, r}\left(\Omega^{\prime}\right)$ by local reduction to $\overline{\mathbb{R}^{n}}$. Correspondingly, we also obtain restriction and extension maps

$$
\gamma_{\partial_{\mathrm{int}} \Omega_{j}}: \bar{H}_{\mathrm{sc}}^{s, r}\left(\Omega_{j}\right) \rightarrow H_{\mathrm{sc}}^{s-1 / 2, r}\left(\partial_{\mathrm{int}} \Omega_{j}\right), \quad e_{\partial_{\mathrm{int}} \Omega_{j}}: H_{\mathrm{sc}}^{s-1 / 2, r}\left(\partial_{\mathrm{int}} \Omega_{j}\right) \rightarrow \bar{H}_{\mathrm{sc}}^{s, r}\left(\Omega_{j}\right) .
$$

With this background we have:

Lemma 4.9. Let $\digamma, \digamma_{0}$ as in Corollary 4.6, and let $k \in \mathbb{R}$.

For $\psi \in H_{\mathrm{sc}}^{1 / 2, k}\left(\partial_{\mathrm{int}} \Omega_{j}\right)$ there is a unique $u \in \bar{H}_{\mathrm{sc}}^{1, k}\left(\Omega_{j}\right)$ such that $\Delta_{\digamma, s} u=0$, $\gamma_{\partial_{\mathrm{int}} \Omega_{j}} u=\psi$.

This defines the Poisson operator $B_{\Omega_{j}}: H_{\mathrm{sc}}^{1 / 2, k}\left(\partial_{\mathrm{int}} \Omega_{j}\right) \rightarrow \bar{H}_{\mathrm{sc}}^{1, k}\left(\Omega_{j}\right)$ solving

$$
\Delta_{\digamma, s} B_{\Omega_{j}}=0, \gamma_{\partial_{\mathrm{int}} \Omega_{j}} B_{\Omega_{j}}=\mathrm{Id},
$$

which has the property that, for $s>1 / 2$, and for $\phi \in C^{\infty}\left(\overline{\Omega_{j}}\right)$ supported away from $\partial_{\mathrm{int}} \Omega_{j}, \phi B_{\Omega_{j}}: H_{\mathrm{sc}}^{s-1 / 2, r}\left(\partial_{\mathrm{int}} \Omega_{j}\right) \rightarrow H_{\mathrm{sc}}^{s, r}\left(\Omega_{j}\right)$.

Proof. The uniqueness follows from the unique solvability of the Dirichlet problem with vanishing boundary conditions, as we already discussed, while the existence by taking $u=e_{\partial_{\mathrm{int}} \Omega_{j}} \psi-\Delta_{\digamma, s}^{-1} \Delta_{\digamma, s} e_{\partial_{\mathrm{int}} \Omega_{j}} \psi$, where $\Delta_{\digamma, s}^{-1}$ is, as before, the inverse of the operator with vanishing Dirichlet boundary conditions. The mapping property also follows from this explicit description, the mapping properties of $e_{\partial_{\mathrm{int}} \Omega_{j}}$ as well 
as Corollary 4.7, since one can arrange that $e_{\partial_{\mathrm{int}} \Omega_{j}}$ maps to distributions supported away from $\operatorname{supp} \phi$.

Let $\Omega_{2}$ be a larger neighborhood of $\Omega$; all of our constructions take place in $\Omega_{2}$. Let $\tilde{\Omega}_{j}=\overline{\Omega_{j}} \backslash \partial_{\mathrm{int}} \Omega_{j}$ (so the artificial boundary is included, but not the interior one). Let $G$ be a parametrix for $A_{\digamma}$ in $\Omega_{2}$; it is thus a scattering ps.d.o. with Schwartz kernel compactly supported in $\tilde{\Omega}_{2} \times \tilde{\Omega}_{2}$. Then $G A_{\digamma}=I+E$, where $\mathrm{WF}_{\mathrm{sc}}^{\prime}(E)$ is disjoint from a neighborhood $\Omega_{1}$ (compactly contained in $\Omega_{2}$ ) of the original region $\Omega$, and $E=-\mathrm{Id}$ near $\partial_{\mathrm{int}} \Omega_{2}$. Now one has

$$
G\left(N_{\digamma}+d_{\digamma}^{s} M \delta_{\digamma}^{s}\right)=I+E,
$$

as operators acting on an appropriate function space on $\Omega_{2}$. We now apply $\mathcal{S}_{\digamma, \Omega_{2}}$ from both sides. Then

$$
N_{\digamma} \mathcal{S}_{\digamma, \Omega_{2}}=N_{\digamma}
$$

since

$$
N_{\digamma} \mathcal{P}_{\digamma, \Omega_{2}}=N_{\digamma} d_{\digamma}^{s} Q_{\digamma, \Omega_{2}}=0
$$

in view of the vanishing boundary condition $Q_{\digamma, \Omega_{2}}$ imposes. On the other hand,

$$
\delta_{\digamma}^{s} \mathcal{S}_{\digamma, \Omega_{2}}=\delta_{\digamma}^{s}-\delta_{\digamma}^{s} d_{\digamma}^{s} Q_{\digamma, \Omega_{2}}=0
$$

so

$$
\mathcal{S}_{\digamma, \Omega_{2}} G N_{\digamma}=\mathcal{S}_{\digamma, \Omega_{2}}+\mathcal{S}_{\digamma, \Omega_{2}} E \mathcal{S}_{\digamma, \Omega_{2}} .
$$

In order to think of this as giving operators on $\Omega_{1}$, let $e_{12}$ be the extension map from $\Omega_{1}$ to $\Omega_{2}$, extending functions (vector fields) as 0 , and $r_{21}$ be the restriction map. (Note that $e_{12}$ correspondingly maps into a relatively low regularity space, such as $L^{2}$, even if one starts with high regularity data.) Then, with the understanding that $N_{\digamma}=N_{\digamma} e_{12}$,

$$
r_{21} \mathcal{S}_{\digamma, \Omega_{2}} G N_{\digamma}=r_{21} \mathcal{S}_{\digamma, \Omega_{2}} e_{12}+K_{1}, \quad K_{1}=r_{21} \mathcal{S}_{\digamma, \Omega_{2}} E \mathcal{S}_{\digamma, \Omega_{2}} e_{12} .
$$

We have:

Lemma 4.10. Let $\digamma, \digamma_{0}$ as in Corollary 4.6.

The operator $K_{1}=r_{21} \mathcal{S}_{\digamma, \Omega_{2}} E \mathcal{S}_{\digamma, \Omega_{2}} e_{12}$ is a smoothing operator in the sense that it maps $x^{k} L_{\mathrm{sc}}^{2}\left(\Omega_{1}\right)$ to $\bar{H}_{\mathrm{sc}}^{s, r}\left(\Omega_{1}\right)$ for every $s, r, k$. Further, for $\psi \in C^{\infty}\left(\overline{\Omega_{2}}\right)$ with support in $\Omega_{1}, \psi K_{1} \psi \in \Psi_{\mathrm{sc}}^{-\infty,-\infty}(X)$.

Further, for any $s, r, k$, given $\epsilon>0$ there exists $\delta>0$ such that if $e_{\delta 1}$ is the exten-

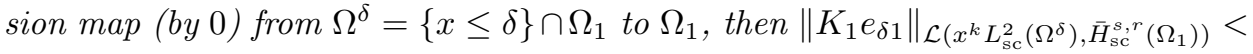
$\epsilon$.

Proof. This follows from Corollary 4.8. Indeed, with $\chi \equiv 1$ near $\partial_{\text {int }} \Omega_{2}$ but with $E=-\mathrm{Id}$ on $\operatorname{supp} \chi$, and with $\phi \in C^{\infty}\left(\overline{\Omega_{2}}\right)$ vanishing near $\operatorname{supp} \chi, \operatorname{supp} \phi \cap$ $\mathrm{WF}_{\mathrm{sc}}^{\prime}(E)=\emptyset, \phi \equiv 1$ near $\overline{\Omega_{1}}$, and with $T$ defined by the first equality,

$$
\begin{aligned}
T=\phi \mathcal{S}_{\digamma, \Omega_{2}} E \mathcal{S}_{\digamma, \Omega_{2}} \phi= & \phi \mathcal{S}_{\digamma, \Omega_{2}} \chi E \chi \mathcal{S}_{\digamma, \Omega_{2}} \phi \\
& +\phi \mathcal{S}_{\digamma, \Omega_{2}}(1-\chi) E \chi \mathcal{S}_{\digamma, \Omega_{2}} \phi \\
& +\phi \mathcal{S}_{\digamma, \Omega_{2}} \chi E(1-\chi) \mathcal{S}_{\digamma, \Omega_{2}} \phi \\
& +\phi \mathcal{S}_{\digamma, \Omega_{2}}(1-\chi) E(1-\chi) \mathcal{S}_{\digamma, \Omega_{2}} \phi .
\end{aligned}
$$

Now, $E(1-\chi) \mathcal{S}_{\digamma, \Omega_{2}} \phi, \phi \mathcal{S}_{\digamma, \Omega_{2}}(1-\chi) E \in \Psi_{\mathrm{sc}}^{-\infty,-\infty}(X)$ since they are in $\Psi_{\mathrm{sc}}^{0,0}(X)$ and $\mathrm{WF}_{\mathrm{sc}}^{\prime}(E) \cap \operatorname{supp} \phi=\emptyset$, so they are smoothing. In combination with Corollary 4.8 this gives that $T: H_{\mathrm{sc}}^{s^{\prime}, r^{\prime}}(X) \rightarrow H_{\mathrm{sc}}^{s, r}(X)$ continuously for all $s, r, s^{\prime}, r^{\prime}$, so composing 
with the extension and restriction maps, noting $r_{21} \phi=r_{21}, \phi e_{12}=e_{12}$, proves the first part of the lemma.

To see the smallness claim, note that

$$
K_{1} e_{\delta 1}=r_{21} T e_{\delta_{1}}=r_{21}\left(T x^{-1}\right)\left(x e_{\delta 2}\right)
$$

$x e_{\delta 2}: x^{k} L_{\mathrm{sc}}^{2}\left(\Omega^{\delta}\right) \rightarrow x^{k} L_{\mathrm{sc}}^{2}\left(\Omega_{1}\right)$ has norm $\leq \sup _{\Omega_{\delta}} x \leq \delta$, while $T x^{-1}: H_{\mathrm{sc}}^{0, k}(X) \rightarrow$ $H_{\mathrm{sc}}^{s, r}(X)$ is bounded, with bound independent of $\delta$, and the same is true for $r_{21}$ : $H_{\mathrm{sc}}^{s, r}(X) \rightarrow \bar{H}_{\mathrm{sc}}^{s, r}\left(\Omega_{2}\right)$, completing the proof.

Now,

$$
\begin{aligned}
\mathcal{S}_{\digamma, \Omega_{1}}-r_{21} \mathcal{S}_{\digamma, \Omega_{2}} e_{12} & =-d_{\digamma}^{s} Q_{\digamma, \Omega_{1}}+r_{21} d_{\digamma}^{s} Q_{\digamma, \Omega_{2}} e_{12} \\
& =-d_{\digamma}^{s} Q_{\digamma, \Omega_{1}}+d_{\digamma}^{s} r_{21} Q_{\digamma, \Omega_{2}} e_{12} \\
& =-d_{\digamma}^{s}\left(Q_{\digamma, \Omega_{1}}-r_{21} Q_{\digamma, \Omega_{2}} e_{12}\right)
\end{aligned}
$$

and with $\gamma_{\partial_{\mathrm{int}} \Omega_{1}}$ denoting the restriction operator to $\partial_{\mathrm{int}} \Omega_{1}$ as above,

$$
\gamma_{\partial_{\mathrm{int}} \Omega_{1}}\left(Q_{\digamma, \Omega_{1}}-r_{21} Q_{\digamma, \Omega_{2}} e_{12}\right)=-\gamma_{\partial_{\mathrm{int}} \Omega_{1}} Q_{\digamma, \Omega_{2}} e_{12}
$$

so

$$
r_{21} \mathcal{S}_{\digamma, \Omega_{2}} G N_{\digamma}=\mathcal{S}_{\digamma, \Omega_{1}}+d_{\digamma}^{s}\left(Q_{\digamma, \Omega_{1}}-r_{21} Q_{\digamma, \Omega_{2}} e_{12}\right)+K_{1} .
$$

Thus, with $B_{\Omega_{1}}$ being the Poisson operator for $\Delta_{\digamma, s}$ on $\Omega_{1}$ as above,

so

$$
\begin{aligned}
r_{21} \mathcal{S}_{\digamma, \Omega_{2}} G N_{\digamma}=\mathcal{S}_{\digamma, \Omega_{1}} & +d_{\digamma}^{s}\left(Q_{\digamma, \Omega_{1}}-r_{21} Q_{\digamma, \Omega_{2}} e_{12}+B_{\Omega_{1}} \gamma_{\partial_{\mathrm{int}} \Omega_{1}} Q_{\digamma, \Omega_{2}} e_{12}\right) \\
& -d_{\digamma}^{s} B_{\Omega_{1}} \gamma_{\partial_{\mathrm{int}} \Omega_{1}} Q_{\digamma, \Omega_{2}} e_{12}+K_{1}
\end{aligned}
$$

$$
\mathcal{S}_{\digamma, \Omega_{1}} r_{21} \mathcal{S}_{\digamma, \Omega_{2}} G N_{\digamma}=\mathcal{S}_{\digamma, \Omega_{1}}-\mathcal{S}_{\digamma, \Omega_{1}} d_{\digamma}^{s} B_{\Omega_{1}} \gamma_{\partial_{\mathrm{int}} \Omega_{1}} Q_{\digamma, \Omega_{2}} e_{12}+\mathcal{S}_{\digamma, \Omega_{1}} K_{1} .
$$

Now we consider applying this to vector fields in $\Omega=\Omega_{0}$, writing $e_{0 j}$ for the extension map to $\Omega_{j}$. Composing from the right,

$$
\mathcal{S}_{\digamma, \Omega_{1}} r_{21} \mathcal{S}_{\digamma, \Omega_{2}} G N_{\digamma}=\mathcal{S}_{\digamma, \Omega_{1}} e_{01}-\mathcal{S}_{\digamma, \Omega_{1}} d_{\digamma}^{s} B_{\Omega_{1}} \gamma_{\partial_{\mathrm{int}} \Omega_{1}} Q_{\digamma, \Omega_{2}} e_{02}+\mathcal{S}_{\digamma, \Omega_{1}} K_{1} e_{01} .
$$

Now:

Lemma 4.11. Let $\digamma, \digamma_{0}$ as in Corollary 4.6 .

The operator $K_{1}^{\prime}=\mathcal{S}_{\digamma, \Omega_{1}} d_{\digamma}^{s} B_{\Omega_{1}} \gamma_{\partial_{\mathrm{int}} \Omega_{1}} Q_{\digamma, \Omega_{2}} e_{02}$ is smoothing in the sense that for $\phi \in C_{c}^{\infty}\left(\overline{\Omega_{1}} \backslash \partial_{\mathrm{int}} \Omega_{1}\right)$,

$$
\phi \mathcal{S}_{\digamma, \Omega_{1}} d_{\digamma}^{s} B_{\Omega_{1}} \gamma_{\partial_{\mathrm{int}} \Omega_{1}} Q_{\digamma, \Omega_{2}} e_{02}: L_{\mathrm{sc}}^{2}(\Omega) \rightarrow H_{\mathrm{sc}}^{s, r}(X)
$$

for all $s, r$, and indeed $\phi \mathcal{S}_{\digamma, \Omega_{1}} d_{\digamma}^{s} B_{\Omega_{1}} \gamma_{\partial_{\mathrm{int}} \Omega_{1}} Q_{\digamma, \Omega_{2}} \phi \in \Psi_{\mathrm{sc}}^{-\infty,-\infty}(X)$.

Further, for any $s, r, k$, given $\epsilon>0$ there exists $\delta>0$ such that if

$$
\Omega \subset \Omega^{\delta}=\{x \leq \delta\} \cap \Omega_{1}
$$

then

$$
\left\|K_{1}^{\prime}\right\|_{\mathcal{L}\left(x^{k} L_{\mathrm{sc}}^{2}(\Omega), \bar{H}_{\mathrm{sc}}^{s, r}\left(\Omega_{1}\right)\right)}<\epsilon .
$$

Proof. By Corollary 4.6, using that $\delta_{\digamma}^{s}$ is a differential operator,

$$
\psi Q_{\digamma, \Omega_{2}} \phi \in \Psi_{\mathrm{sc}}^{-\infty,-\infty}(X)
$$

whenever $\psi, \phi \in C^{\infty}\left(\overline{\Omega_{2}}\right)$ have disjoint supports, also disjoint from $\partial_{\text {int }} \Omega_{2}$ since this operator is in $\Psi_{\mathrm{sc}}^{-1,0}(X)$ directly from the corollary, and then the disjointness of supports gives the conclusion. Taking such $\psi, \phi$, as one may, with $\phi \equiv 1$ near $\bar{\Omega}$, while $\psi \equiv 1$ near $\partial_{\text {int }} \Omega_{1}$, we see that $\gamma_{\partial_{\text {int }} \Omega_{1}} Q_{\digamma, \Omega_{2}} e_{02}: x^{k} L_{\mathrm{sc}}^{2}(\Omega) \rightarrow H_{\mathrm{sc}}^{s, r}\left(\partial_{\mathrm{int}} \Omega_{1}\right)$ for all $s, r, k$, i.e. mapping to $\dot{C}^{\infty}\left(\partial_{\text {int }} \Omega_{1}\right)$. The first part then follows from $B_{\Omega_{1}}$ 
mapping this to $\bar{H}_{\mathrm{sc}}^{1, r}\left(\Omega_{1}\right)$ for all $r$, with the additional property that $\tilde{\phi} B_{\Omega_{1}}$ maps to $H_{\mathrm{sc}}^{s, r}(\Omega)$ for all $s, r$ if $\tilde{\phi}$ has properties like $\phi$, and then Corollary 4.8 completes the argument.

For the smallness, we just need to proceed as in Lemma 4.10, writing

$$
\gamma_{\partial_{\mathrm{int}} \Omega_{1}} Q_{\digamma, \Omega_{2}} e_{02}=\gamma_{\partial_{\mathrm{int}} \Omega_{1}}\left(\psi Q_{\digamma, \Omega_{2}} \phi x^{-1}\right)\left(x e_{02}\right),
$$

where now $\psi Q_{\digamma, \Omega_{2}} \phi x^{-1} \in \Psi_{\mathrm{sc}}^{-\infty,-\infty}(X)$, thus bounded between all weighted Sobolev spaces, with norm independent of $\delta$, while $x e_{02}: x^{k} L_{\mathrm{sc}}^{2}\left(\Omega^{\delta}\right) \rightarrow x^{k} L_{\mathrm{sc}}^{2}\left(\Omega_{2}\right)$ has norm $\leq \delta$

Thus,

$$
\mathcal{S}_{\digamma, \Omega_{1}} r_{21} \mathcal{S}_{\digamma, \Omega_{2}} G N_{\digamma}=\mathcal{S}_{\digamma, \Omega_{1}} e_{01}+K_{2},
$$

with $K_{2}$ smoothing and small if $\Omega \subset\{x \leq \delta\}$, with $\delta$ suitably small. This is exactly Equation (5.7) of [24, and from this point on we can follow the argument of the global work of Stefanov and Uhlmann [24, Section 5], with the addition of having a small rather than just compact error, giving invertibility.

Restricting to $\Omega$ from the left, the key remaining step is to compute $\mathcal{S}_{\digamma, \Omega}-$ $r_{10} \mathcal{S}_{\digamma, \Omega_{1}} e_{01}$ in terms of the already existing information. As above,

$$
\mathcal{S}_{\digamma, \Omega}-r_{10} \mathcal{S}_{\digamma, \Omega_{1}} e_{01}=-d_{\digamma}^{s}\left(Q_{\digamma, \Omega}-r_{10} Q_{\digamma, \Omega_{1}} e_{01}\right),
$$

but now we compute $u=\left(Q_{\digamma, \Omega}-r_{10} Q_{\digamma, \Omega_{1}} e_{01}\right) f$ using that it is the solution of the Dirichlet problem $\Delta_{\digamma, s} u=0, \gamma_{\partial_{\mathrm{int}} \Omega} u=-\gamma_{\partial_{\mathrm{int} \Omega} \Omega} Q_{\digamma, \Omega_{1}} e_{01} f$, so

$$
u=-B_{\Omega} \gamma_{\partial_{\mathrm{int}} \Omega} Q_{\digamma, \Omega_{1}} e_{01} f,
$$

and using that one can compute $\gamma_{\partial_{\text {int }} \Omega} Q_{\digamma, \Omega_{1}} e_{01} f$ from $d_{\digamma}^{s} Q_{\digamma, \Omega_{1}} e_{01} f$. Concretely, we have the following lemma on functions:

Lemma 4.12. Let $\dot{H}_{\mathrm{sc}}^{1,0}\left(\Omega_{1} \backslash \Omega\right)$ denote the restriction of elements of $\dot{H}_{\mathrm{sc}}^{1,0}\left(\Omega_{1}\right)$ to $\Omega_{1} \backslash \bar{\Omega}$ (thus, these need not vanish at $\partial_{\text {int }} \Omega$ ), and let $\rho_{\Omega_{1} \backslash \Omega}$ be a defining function of $\partial_{\mathrm{int}} \Omega$ as a boundary of $\Omega_{1} \backslash \Omega$, i.e. it is positive in the latter set. Suppose that $\partial_{x} \rho_{\Omega_{1} \backslash \Omega}>0$ at $\partial_{\text {int }} \Omega$; note that this is independent of the choice of $\rho_{\Omega_{1} \backslash \Omega}$ satisfying the previous criteria (so this is a statement on $x$ being increasing as one leaves $\Omega$ at $\partial_{\mathrm{int}} \Omega$ ). Then on functions, for $\digamma>0, k \in \mathbb{R}$, the map

$$
d_{\digamma}^{s}: \dot{H}_{\mathrm{sc}}^{1, k}\left(\Omega_{1} \backslash \Omega\right) \rightarrow x^{k} L^{2}\left(\Omega_{1} \backslash \Omega\right)
$$

is injective, with a continuous left inverse $P_{\Omega_{1} \backslash \Omega}: x^{k} L^{2}\left(\Omega_{1} \backslash \Omega\right) \rightarrow \dot{H}_{\mathrm{sc}}^{1, k}\left(\Omega_{1} \backslash \Omega\right)$.

Proof. Consider $k=0$ first.

The norm of $d_{\digamma}^{s} u$ is certainly equivalent to that of $\nabla u$ in $L^{2}\left(\Omega_{1} \backslash \Omega\right)$ modulo the $L^{2}\left(\Omega_{1} \backslash \Omega\right)$ norm of $u$, so one only needs to prove a local Poincaré inequality

$$
\|u\|_{L^{2}\left(\Omega_{1} \backslash \Omega\right)} \leq C\left\|d_{\digamma}^{s} u\right\|_{L^{2}\left(\Omega_{1} \backslash \Omega\right)}
$$

to conclude that

$$
\|u\|_{\dot{H}_{\mathrm{sc}}^{1,0}\left(\Omega_{1} \backslash \Omega\right)} \leq C\left\|d_{\digamma}^{s} u\right\|_{L^{2}\left(\Omega_{1} \backslash \Omega\right)}
$$

which proves the lemma in this case, since it proves that $d_{\digamma}^{s}$, between these spaces, has closed range and is injective, so it is an isomorphism between $\dot{H}_{\mathrm{sc}}^{1,0}\left(\Omega_{1} \backslash \Omega\right)$ and its range, and then its inverse in this sense can be extended continuously to $L^{2}\left(\Omega_{1} \backslash \Omega\right)$.

But 4.14 can be proved similarly to Lemma 4.2, by showing that

$$
\|u\|_{L^{2}\left(\Omega_{1} \backslash \Omega\right)} \leq C\left\|\left(x^{2} D_{x}+i \digamma\right) u\right\|_{L^{2}\left(\Omega_{1} \backslash \Omega\right)} .
$$


Here we want to use $P=x^{2} D_{x}+i \digamma$ and $\|P u\|^{2}$ again; we need to be careful at $\partial_{\text {int }} \Omega$ since $u$ does not vanish there. Thus, there is an integration by parts boundary term, which we express in terms of the characteristic function $\chi_{\Omega_{1} \backslash \Omega}$ :

$$
\begin{aligned}
\|P u\|_{L^{2}\left(\Omega_{1} \backslash \Omega\right)}^{2} & =\left\langle\chi_{\Omega_{1} \backslash \Omega} P u, P u\right\rangle_{L^{2}\left(\Omega_{1}\right)}=\left\langle P^{*} \chi_{\Omega_{1} \backslash \Omega} P u, u\right\rangle_{L^{2}\left(\Omega_{1}\right)} \\
& =\left\langle P^{*} P u, u\right\rangle_{L^{2}\left(\Omega_{1} \backslash \Omega\right)}+\left\langle\left[P^{*}, \chi_{\Omega_{1} \backslash \Omega} P u, u\right\rangle_{L^{2}\left(\Omega_{1}\right)} .\right.
\end{aligned}
$$

Similarly,

$$
\left\|P_{R} u\right\|_{L^{2}\left(\Omega_{1} \backslash \Omega\right)}^{2}=\left\langle P_{R}^{*} P_{R} u, u\right\rangle_{L^{2}\left(\Omega_{1} \backslash \Omega\right)}+\left\langle\left[P_{R}^{*}, \chi_{\Omega_{1} \backslash \Omega}\right] P_{R} u, u\right\rangle_{L^{2}\left(\Omega_{1}\right)} .
$$

On the other hand, with $P_{I}$ being 0 th order, the commutator term vanishes for it. Correspondingly,

$$
\begin{aligned}
\|P u\|_{L^{2}\left(\Omega_{1} \backslash \Omega\right)}^{2}= & \left\langle P^{*} P u, u\right\rangle_{L^{2}\left(\Omega_{1} \backslash \Omega\right)}+\left\langle\left[P^{*}, \chi_{\Omega_{1} \backslash \Omega}\right] P u, u\right\rangle_{L^{2}\left(\Omega_{1}\right)} \\
= & \left\langle P_{R}^{*} P_{R} u, u\right\rangle_{L^{2}\left(\Omega_{1} \backslash \Omega\right)}+\left\langle P_{I}^{*} P_{I} u, u\right\rangle_{L^{2}\left(\Omega_{1} \backslash \Omega\right)}+\left\langle i\left[P_{R}, P_{I}\right] u, u\right\rangle_{L^{2}\left(\Omega_{1} \backslash \Omega\right)} \\
& \quad+\left\langle\left[P^{*}, \chi_{\Omega_{1} \backslash \Omega}\right] P u, u\right\rangle_{L^{2}\left(\Omega_{1}\right)} \\
= & \left\|P_{R} u\right\|_{L^{2}\left(\Omega_{1} \backslash \Omega\right)}^{2}+\left\|P_{I} u\right\|_{L^{2}\left(\Omega_{1} \backslash \Omega\right)}^{2}+\left\langle i\left[P_{R}, P_{I}\right] u, u\right\rangle_{L^{2}\left(\Omega_{1} \backslash \Omega\right)} \\
& \quad+\left\langle\left[P^{*}, \chi_{\Omega_{1} \backslash \Omega}\right] P u, u\right\rangle_{L^{2}\left(\Omega_{1}\right)}-\left\langle\left[P_{R}^{*}, \chi_{\Omega_{1} \backslash \Omega}\right] P_{R} u, u\right\rangle_{L^{2}\left(\Omega_{1}\right)} .
\end{aligned}
$$

Now, as $P-P_{R}$ is 0th order, $\left[P^{*}, \chi_{\Omega_{1} \backslash \Omega}\right]=\left[P_{R}^{*}, \chi_{\Omega_{1} \backslash \Omega}\right]$, so the last two terms on the right hand side give

$$
\left\langle\left[P^{*}, \chi_{\Omega_{1} \backslash \Omega}\right] i P_{I} u, u\right\rangle_{L^{2}\left(\Omega_{1}\right)}=\left\langle x^{2} \partial_{x} \chi_{\Omega_{1} \backslash \Omega}\left(\digamma-\frac{n-1}{2} x\right) u, u\right\rangle_{L^{2}\left(\Omega_{1}\right)},
$$

which is non-negative, at least if $x$ is sufficiently small (or $\digamma$ large) on $\partial_{\text {int }} \Omega$ since $\chi_{\Omega_{1} \backslash \Omega}=\chi_{(0, \infty)} \circ \rho_{\Omega_{1} \backslash \Omega}$. Correspondingly, this term can be dropped, and one obtains 4.15 at least if $x$ is small on $\Omega_{1}$ just as in the proof of Lemma 4.2. The case of $x$ not necessarily small on $\Omega_{1}$ (though small on $\Omega$ ) follows exactly as in Lemma 4.2 using the standard Poincaré inequality, and even the case where $x$ is not small on $\Omega$ can be handled similarly since one now has an extra term at $\partial_{\text {int }} \Omega$, away from $x=0$, which one can control using the standard Poincaré inequality. This gives

$$
\|u\|_{\dot{H}_{\mathrm{sc}}^{1,0}\left(\Omega_{1} \backslash \Omega\right)} \leq C\left\|d_{\digamma}^{s} u\right\|_{L^{2}\left(\Omega_{1} \backslash \Omega\right)}
$$

showing the claimed injectivity. Further, this gives a continuous inverse from the range of $d_{\digamma}^{s}$, which is closed in $L^{2}\left(\Omega_{1} \backslash \Omega\right)$; one can use an orthogonal projection to this space to define the left inverse $P_{\Omega_{1} \backslash \Omega}$, completing the proof when $k=0$.

For general $k$, one can proceed as in Lemma 4.4, conjugating $d_{\digamma}^{s}$ by $x^{k}$, which changes it by $x$ times a smooth one form; this changes $x^{2} D_{x}+i \digamma$ by an element of $x C^{\infty}(X)$, with the only effect of modifying the $x \frac{n-1}{2}$ term in 4.16$)$, which does not affect the proof.

We now turn to one forms.

Lemma 4.13. Let $\dot{H}_{\mathrm{sc}}^{1,0}\left(\Omega_{1} \backslash \Omega\right)$ be as in Lemma 4.12, but with values in oneforms, and let $\rho_{\Omega_{1} \backslash \Omega}$ be a defining function of $\partial_{\mathrm{int}} \Omega$ as a boundary of $\Omega_{1} \backslash \Omega$, i.e. it is positive in the latter set. Suppose that $\partial_{x} \rho_{\Omega_{1} \backslash \Omega}>0$ at $\partial_{\text {int }} \Omega$; note that this is independent of the choice of $\rho_{\Omega_{1} \backslash \Omega}$ satisfying the previous criteria (so this is a statement on $x$ being increasing as one leaves $\Omega$ at $\left.\partial_{\text {int }} \Omega\right)$. Then for $r \leq-(n-5) / 2$, on one-forms the map

$$
d_{\digamma}^{s}: \dot{H}_{\mathrm{sc}}^{1, r}\left(\Omega_{1} \backslash \Omega\right) \rightarrow H_{\mathrm{sc}}^{0, r}\left(\Omega_{1} \backslash \Omega\right)
$$


is injective, with a continuous left inverse $P_{\Omega_{1} \backslash \Omega}: H_{\mathrm{sc}}^{0, r}\left(\Omega_{1} \backslash \Omega\right) \rightarrow \dot{H}_{\mathrm{sc}}^{1, r-2}\left(\Omega_{1} \backslash \Omega\right)$.

Remark 4.14. Unfortunately the argument given above for functions would give an unfavorable boundary term, so instead we proceed proving the local Poincaré inequality directly and using our generalized Korn's inequality, Lemma 4.5, to avoid a loss of derivatives. However, our method still produces a loss of weight, essentially because as presented the estimate would be natural for standard tensors, not scattering tensors, hence the presence of the loss -2 in the weight in the statement of the lemma.

Proof. As in the work of the first two authors, [23, Section 6], we prove the Poincaré inequality using the identity, see [18, Chapter 3.3],

$$
\sum_{i}[v(\gamma(s))]_{i} \dot{\gamma}^{i}(s)=\int_{0}^{s} \sum_{i j}\left[d^{s} v(\gamma(t))\right]_{i j} \dot{\gamma}^{i}(t) \dot{\gamma}^{j}(t) d t
$$

where $\gamma$ is a unit speed geodesic of the original metric $g$ (thus not of a scattering metric) with $\gamma(0) \in \partial_{\text {int }} \Omega_{1}$ (so $v(\gamma(0))$ vanishes) and $\gamma(\tau) \in \partial_{\text {int }} \Omega \cup \partial X$, with $\left.\gamma\right|_{(0, \tau)}$ in $\Omega_{1} \backslash \bar{\Omega}$. Identity 4.17$)$ is just an application of the Fundamental Theorem of Calculus with the $s$-derivative of the l.h.s. computed using the rules of covariant differentiation. In this formula we use $\left[d^{s} v(\gamma(t))\right]_{i j}$ for the components in the symmetric 2-cotensors corresponding to the standard cotangent bundle, and similarly for $[v(\gamma(s))]_{i}$. Notice that this formula gives an explicit left inverse for $d_{\digamma}^{s}$, as discussed below.

Here we choose $\gamma$ such that $x \circ \gamma$ is strictly monotone in the sense that $-\frac{\partial}{\partial t}(x \circ \gamma)$ is bounded below (and above) by a positive constant, thus $(x \circ \gamma)^{2} \frac{\partial}{\partial t}\left(x^{-1} \circ \gamma\right)$ has the same property. Note that one can construct a smooth family of such geodesics emanating from $\partial_{\text {int }} \Omega_{1}$, parameterized by $\partial_{\text {int }} \Omega$, in a manner that, with $d \omega$ a smooth measure on $\partial_{\text {int }} \Omega_{1}, d \omega d t$ is equivalent to the volume form $d g$, i.e. also to $d x d y_{1} \ldots d y_{n-1}$. Thus, for any $k \geq 0$, using $x(\gamma(s)) \leq x(\gamma(t))$ along the geodesic segment, $t \in[0, s]$,

$$
\begin{aligned}
&\left|e^{-\digamma / x(\gamma(s))} x(\gamma(s))^{k} \sum_{i}[v(\gamma(s))]_{i} \dot{\gamma}^{i}(s)\right|^{2} \\
&=\mid \int_{0}^{s} \sum_{i j} e^{-\digamma / x(\gamma(t))} x(\gamma(t))^{k+1}\left[d^{s} v(\gamma(t))\right]_{i j} \dot{\gamma}^{i}(t) \dot{\gamma}^{j}(t) \times\left. e^{-\digamma(1 / x(\gamma(s))-1 / x(\gamma(t)))} x(\gamma(t))^{-1} d t\right|^{2} \\
& \leq n^{2}\left(\int_{0}^{\tau} \sum_{i j} e^{-2 \digamma / x(\gamma(t))} x(\gamma(t))^{2 k+2}\left|\left[d^{s} v(\gamma(t))\right]_{i j} \dot{\gamma}^{i}(t) \dot{\gamma}^{j}(t)\right|^{2} d t\right) \\
& \times\left(\int_{0}^{s} e^{-2 \digamma(1 / x(\gamma(s))-1 / x(\gamma(t)))} x(\gamma(t))^{-2} d t\right) .
\end{aligned}
$$


Thus,

$$
\begin{aligned}
& \left|e^{-\digamma / x(\gamma(s))} x(\gamma(s))^{k} \sum_{i}[v(\gamma(s))]_{i} \dot{\gamma}^{i}(s)\right|^{2} \\
& \leq C^{\prime}\left(\int_{0}^{\tau} \sum_{i j} e^{-2 \digamma / x(\gamma(t))} x(\gamma(t))^{2 k+2}\left|\left[d^{s} v(\gamma(t))\right]_{i j} \dot{\gamma}^{i}(t) \dot{\gamma}^{j}(t)\right|^{2} d t\right) \\
& \times\left(\int_{0}^{s} e^{-2 \digamma(1 / x(\gamma(s))-1 / x(\gamma(t)))}\left(-\frac{\partial}{\partial t}\left(x^{-1}(\gamma(t))\right)\right) d t\right) \\
& \leq C^{\prime}\left(\int_{0}^{\tau} e^{-2 \digamma / x(\gamma(t))} x(\gamma(t))^{2 k+2}\left|d^{s} v(\gamma(t))\right|_{\ell^{2}}^{2} d t\right)\left(\int_{r_{0}}^{x^{-1}(\gamma(s))} e^{-2 \digamma(1 / x(\gamma(s))-r)} d r\right)
\end{aligned}
$$

for suitable $r_{0}>0$, where we wrote $r=x^{-1}$, and we used the lower bound for $(x \circ \gamma)^{2} \frac{\partial}{\partial t}\left(x^{-1} \circ \gamma\right)$ in the second factor, and that $\gamma$ is unit speed in the first factor, with $\ell^{2}$ being the norm as a symmetric map on $T_{p} X$. The second factor on the right hand side is bounded by $(2 \digamma)^{-1}$, so can be dropped. Now, as $\tau d x+\zeta d y=$ $\left(x^{2} \tau\right) \frac{d x}{x^{2}}+(x \zeta) \frac{d y}{x}$, so e.g. the $d x^{2}$ component of $d^{s} v$ is $x^{-4}$ times the $\frac{d x^{2}}{x^{4}}$ component in the scattering basis, we have

$$
\left|d^{s} v(\gamma(t))\right|_{\ell^{2}} \leq C x(\gamma(t))^{-4}\left|d^{s} v(\gamma(t))\right|_{\ell_{\mathrm{sc}}^{2}},
$$

so the right hand side is bounded from above by

$$
C^{\prime \prime} \digamma^{-1} \int_{0}^{\tau} e^{-2 \digamma / x(\gamma(t))} x(\gamma(t))^{2 k-6}\left|d^{s} v(\gamma(t))\right|_{\ell_{\mathrm{sc}}^{2}}^{2} d t
$$

Integrating in the spatial variable, $\gamma(0) \in \partial_{\mathrm{int}} \Omega_{1}$, and using that the second factor is $(2 \digamma)^{-1}$, gives

$$
\left\|e^{-\digamma / x} x^{k} v\left(\gamma^{\prime}\right)\right\|_{L^{2}\left(\Omega_{1} \backslash \Omega\right)}^{2} \leq C \digamma^{-1}\left\|x^{k-3} e^{-\digamma / x} d^{s} v\right\|_{L^{2}\left(\Omega_{1} \backslash \Omega ; \operatorname{Sym}^{2 \mathrm{sc}} T^{*} X\right)}^{2} .
$$

Using different families of geodesics with tangent vectors covering $T X$ over $\Omega_{1} \backslash \Omega$,

$$
\left\|e^{-\digamma / x} x^{k} v\right\|_{L^{2}\left(\Omega_{1} \backslash \Omega ; T^{*} X\right)}^{2} \leq C \digamma^{-1}\left\|x^{k-3} e^{-\digamma / x} d^{s} v\right\|_{L^{2}\left(\Omega_{1} \backslash \Omega ; \mathrm{Sym}^{2 \mathrm{sc}} T^{*} X\right)}^{2} .
$$

Now, similarly to (4.18), but going the opposite direction,

$$
\|v(p)\|_{\ell_{\mathrm{sc}}^{2}} \leq x(p)\|v(p)\|_{\ell^{2}}
$$

so

$$
\left\|e^{-\digamma / x} x^{k-1} v\right\|_{L^{2}\left(\Omega_{1} \backslash \Omega ; \mathrm{sc} T^{*} X\right)}^{2} \leq C \digamma^{-1}\left\|x^{k-3} e^{-\digamma / x} d^{s} v\right\|_{L^{2}\left(\Omega_{1} \backslash \Omega ; \mathrm{Sym}^{2 \mathrm{sc}} T^{*} X\right)}^{2} .
$$

Changing the volume form as well yields

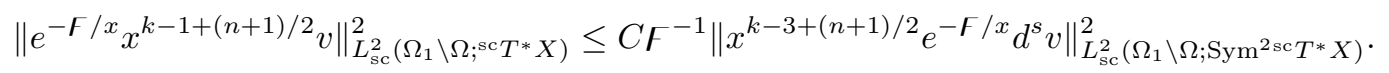

With $u=e^{-\digamma / x} v$, this gives, for $u \in C^{\infty}\left(\overline{\Omega_{1} \backslash \Omega}\right)$, vanishing at $\partial_{\text {int }} \Omega_{1}$, of compact support,

$$
\|u\|_{H_{\mathrm{sc}}^{0, r-2}\left(\Omega_{1} \backslash \Omega\right)}^{2} \leq C \digamma^{-1}\left\|d_{\digamma}^{s} u\right\|_{H_{\mathrm{sc}}^{0, r}\left(\Omega_{1} \backslash \Omega\right)}^{2},
$$

$r \leq-(n-5) / 2$, which then gives the same conclusion, by density and continuity considerations for $u \in \dot{H}_{\mathrm{sc}}^{1, r}\left(\Omega_{1} \backslash \Omega\right)$, the desired Poincaré estimate.

To obtain the $H^{1}$ estimate, we use Lemma 4.5, which gives, even for $u \in$ $\bar{H}_{\mathrm{sc}}^{1, r-2}\left(\Omega_{1} \backslash \Omega\right)$,

$$
\|u\|_{\bar{H}_{\mathrm{sc}}^{1, r-2}\left(\Omega_{1} \backslash \Omega\right)}^{2} \leq C\left(\left\|d_{\digamma}^{s} u\right\|_{H_{\mathrm{sc}}^{0, r-2}\left(\Omega_{1} \backslash \Omega\right)}^{2}+\|u\|_{H_{\mathrm{sc}}^{0, r-2}\left(\Omega_{1} \backslash \Omega\right)}^{2}\right),
$$


which combined with 4.19 proves

$$
\|u\|_{\dot{H}_{\mathrm{sc}}^{1, r-2}\left(\Omega_{1} \backslash \Omega\right)} \leq C\left\|d_{\digamma}^{s} u\right\|_{H_{\mathrm{sc}}^{0, r}\left(\Omega_{1} \backslash \Omega\right)}, \quad u \in \dot{H}_{\mathrm{sc}}^{1, r}\left(\Omega_{1} \backslash \Omega\right),
$$

where recall that our notation is that membership of $\dot{H}_{\mathrm{sc}}^{1, r}\left(\Omega_{1} \backslash \Omega\right)$ only implies vanishing at $\partial_{\text {int }} \Omega_{1}$, not at $\partial_{\text {int }} \Omega$.

Taking into account the above considerations, namely choosing several families of geodesics to span the tangent space, and working with $v=e^{F / x} u$, the formula (4.17) then also gives an explicit formula for the left inverse.

Recall now 4.13):

$$
u=-B_{\Omega} \gamma_{\partial_{\mathrm{int}} \Omega} Q_{\digamma, \Omega_{1}} e_{01} f .
$$

Using Lemmas 4.12 4.13, we conclude that

$$
u=-B_{\Omega} \gamma_{\partial_{\mathrm{int}} \Omega} P_{\Omega_{1} \backslash \Omega} d_{\digamma}^{s} Q_{\digamma, \Omega_{1}} e_{01} f,
$$

and as $e_{01} f$ vanishes on $\Omega_{1} \backslash \Omega$,

$$
\left.\mathcal{S}_{\digamma, \Omega_{1}} e_{01} f\right|_{\Omega_{1} \backslash \Omega}=-\left.d_{\digamma}^{s} Q_{\digamma, \Omega_{1}} e_{01} f\right|_{\Omega_{1} \backslash \Omega},
$$

so

and thus

$$
u=B_{\Omega} \gamma_{\partial_{\mathrm{int}} \Omega} P_{\Omega_{1} \backslash \Omega} \mathcal{S}_{\digamma, \Omega_{1}} e_{01} f
$$

$$
\mathcal{S}_{\digamma, \Omega}-r_{10} \mathcal{S}_{\digamma, \Omega_{1}} e_{01}=-d_{\digamma}^{s} B_{\Omega} \gamma_{\partial_{\mathrm{int}} \Omega} P_{\Omega_{1} \backslash \Omega} \mathcal{S}_{\digamma, \Omega_{1}} e_{01}
$$

Using 4.12 this gives

$$
r_{10} \mathcal{S}_{\digamma, \Omega_{1}} r_{21} \mathcal{S}_{\digamma, \Omega_{2}} G N_{\digamma}=\mathcal{S}_{\digamma, \Omega}+d_{\digamma}^{s} B_{\Omega} \gamma_{\partial_{\text {int }} \Omega} P_{\Omega_{1} \backslash \Omega} \mathcal{S}_{\digamma, \Omega_{1}} e_{01}+r_{10} K_{2} .
$$

Using 4.12 again to express $\mathcal{S}_{\digamma, \Omega_{1}} e_{01}$ on the right hand side, we get

$$
\begin{aligned}
& r_{10} \mathcal{S}_{\digamma, \Omega_{1}} r_{21} \mathcal{S}_{\digamma, \Omega_{2}} G N_{\digamma} \\
& \quad=\mathcal{S}_{\digamma, \Omega}+d_{\digamma}^{s} B_{\Omega} \gamma_{\partial_{\mathrm{int}} \Omega} P_{\Omega_{1} \backslash \Omega}\left(\mathcal{S}_{\digamma, \Omega_{1}} r_{21} \mathcal{S}_{\digamma, \Omega_{2}} G N_{\digamma}-K_{2}\right)+r_{10} K_{2},
\end{aligned}
$$

which gives

$$
\begin{array}{r}
\left(r_{10}-d_{\digamma}^{s} B_{\Omega} \gamma_{\partial_{\mathrm{int}} \Omega} P_{\Omega_{1} \backslash \Omega}\right) \mathcal{S}_{\digamma, \Omega_{1}} r_{21} \mathcal{S}_{\digamma, \Omega_{2}} G N_{\digamma} \\
=\mathcal{S}_{\digamma, \Omega}+\left(r_{10}-d_{\digamma}^{s} B_{\Omega} \gamma_{\partial_{\mathrm{int}} \Omega} P_{\Omega_{1} \backslash \Omega}\right) K_{2} .
\end{array}
$$

We now add $\mathcal{P}_{\digamma, \Omega}$ to both sides, and use that the smallness of $K_{2}$ when $\Omega$ is small enough gives that $\mathrm{Id}+\left(r_{10}-d_{\digamma}^{s} B_{\Omega} \gamma_{\partial_{\text {int }} \Omega} P_{\Omega_{1} \backslash \Omega}\right) K_{2}$ is invertible. Here we need to be careful in the 2-tensor case: while $K_{2}$ is smoothing, including in the sense of producing additional decay, so there is no problem with applying $P_{\Omega_{1} \backslash \Omega}$ regardless of the weighted space we are considering, the result will have only a weighted estimate in $H_{\mathrm{sc}}^{1, r-2}, r \leq-(n-5) / 2$, corresponding to Lemma 4.13 , so the inversion has to be done in a sufficiently negatively weighted space, namely $H_{\mathrm{sc}}^{0, r}(\Omega)$, with $r \leq-(n-1) / 2$. Thus,

$$
\begin{aligned}
& \left(\mathrm{Id}+\left(r_{10}-d_{\digamma}^{s} B_{\Omega} \gamma_{\partial_{\mathrm{int}} \Omega} P_{\Omega_{1} \backslash \Omega}\right) K_{2}\right)^{-1} \\
& \quad \circ\left(\left(r_{10}-d_{\digamma}^{s} B_{\Omega} \gamma_{\partial_{\mathrm{int}} \Omega} P_{\Omega_{1} \backslash \Omega}\right) \mathcal{S}_{\digamma, \Omega_{1}} r_{21} \mathcal{S}_{\digamma, \Omega_{2}} G N_{\digamma}+\mathcal{P}_{\digamma, \Omega}\right)=\mathrm{Id},
\end{aligned}
$$

and so multiplying from $\mathcal{S}_{\digamma, \Omega}$ from the right yields

$$
\begin{aligned}
\left(\mathrm{Id}+\left(r_{10}\right.\right. & \left.\left.-d_{\digamma}^{s} B_{\Omega} \gamma_{\partial_{\mathrm{int}} \Omega} P_{\Omega_{1} \backslash \Omega}\right) K_{2}\right)^{-1} \\
& \circ\left(r_{10}-d_{\digamma}^{s} B_{\Omega} \gamma_{\partial_{\mathrm{int}} \Omega} P_{\Omega_{1} \backslash \Omega}\right) \mathcal{S}_{\digamma, \Omega_{1}} r_{21} \mathcal{S}_{\digamma, \Omega_{2}} G N_{\digamma}=\mathcal{S}_{\digamma, \Omega} .
\end{aligned}
$$

Now recall that $N_{\digamma}=e^{-\digamma / x} L I e^{\digamma / x}$, and that for $f \in e^{\digamma / x} L_{\mathrm{sc}}^{2}(\Omega), \mathcal{P}_{\digamma, \Omega} e^{-\digamma / x} f=0$ amounts to $e^{\digamma / x} \delta^{s} e^{-\digamma / x}\left(e^{-\digamma / x} f\right)=0$, i.e. $\delta^{s}\left(e^{-2 \digamma / x} f\right)=0$. This in particular 
gives an inversion formula for the geodesic $\mathrm{X}$-ray transform on $e^{2 \digamma / x}$-solenoidal one-forms and symmetric 2-tensors.

In order to state the stability estimate it is convenient to consider $(x, y, \lambda, \omega) \in$ $S X$ to actually lie in ${ }^{\text {sc }} S X$ via the identification (multiplying the tangent vector by $x$ )

$$
\left(x, y, \lambda \partial_{x}+\omega \partial_{y}\right) \mapsto\left(x, y,(\lambda / x)\left(x^{2} \partial_{x}\right)+\omega\left(x \partial_{y}\right)\right)
$$

Here ${ }^{\text {sc }} S X=\left({ }^{\mathrm{sc}} T X \backslash o\right) / \mathbb{R}^{+}$is the sphere bundle in ${ }^{\mathrm{sc}} T X$, and in the relevant open set the fiber over a fixed point $(x, y)$ can be identified with vectors of the form $\tilde{\lambda}\left(x^{2} \partial_{x}\right)+\tilde{\omega}\left(x \partial_{y}\right), \tilde{\omega} \in \mathbb{S}^{n-2}, \tilde{\lambda} \in \mathbb{R}$. Then the region $|\lambda / x|<M$ in $S X$ corresponds to the region $|\tilde{\lambda}|<M$; this is now an open subset of ${ }^{\mathrm{sc}} S X$. Note that in particular that the 'blow-down map' $(x, y, \tilde{\lambda}, \tilde{\omega}) \mapsto(x, y, x \tilde{\lambda}, \tilde{\omega})$ is smooth, and the composite map $(x, y, \tilde{\lambda}, \tilde{\omega}, t) \mapsto \gamma_{x, y, x \tilde{\lambda}, \tilde{\omega}}(t)$ has surjective differential. In particular, with

$$
U=\{|\tilde{\lambda}|<M\}
$$

the scattering Sobolev spaces are just restrictions to a domain with smooth boundary. Note that $U$ lies within the set of $\Omega$-local geodesics; we choose $M$ so that supp $\chi \subset M$.

This discussion, in particular 4.20, proves our main local result, for which we reintroduce the subscript $c$ for the size of the region $\Omega_{c}$ :

Theorem 4.15. For one forms, let $\digamma>0$; for symmetric 2-tensors let $\digamma_{0}>0$ be the maximum of the two constants, denoted there by $\digamma_{0}$, in Proposition 3.3 and Corollary 4.6 .

For $\Omega=\Omega_{c}, c>0$ small, the geodesic $X$-ray transform on $e^{2 \digamma / x}$-solenoidal oneforms and symmetric 2-tensors $f \in e^{\digamma / x} L_{\mathrm{sc}}^{2}(\Omega)$, i.e. ones satisfying $\delta^{s}\left(e^{-2 \digamma / x} f\right)=$ 0 , is injective, with a stability estimate and a reconstruction formula

$$
\begin{aligned}
f=e^{\digamma / x}\left(\mathrm{Id}+\left(r_{10}-d_{\digamma}^{s} B_{\Omega} \gamma_{\partial_{\mathrm{int}} \Omega} P_{\Omega_{1} \backslash \Omega}\right) K_{2}\right)^{-1}\left(r_{10}\right. & \left.-d_{\digamma}^{s} B_{\Omega} \gamma_{\partial_{\mathrm{int}} \Omega} P_{\Omega_{1} \backslash \Omega}\right) \\
& \circ \mathcal{S}_{\digamma, \Omega_{1}} r_{21} \mathcal{S}_{\digamma, \Omega_{2}} G e^{-\digamma / x} L I f .
\end{aligned}
$$

Here stability is in the sense that for $s \geq 0$ there exist $R, R^{\prime}$ such that for any (sufficiently negative in the case of 2-tensors) $r$ the $e^{\digamma / x} H_{\mathrm{sc}}^{s-1, r}$ norm of $f$ on $\Omega$ is controlled by the $e^{\digamma / x} H_{\mathrm{sc}}^{s, r+R}$ norm of If on $U$, provided $f$ is a priori in $e^{\digamma / x} H_{\mathrm{sc}}^{s, r+R^{\prime}}$. In addition, replacing $\Omega_{c}=\{\tilde{x}>-c\} \cap M$ by $\Omega_{\tau, c}=\{\tau>\tilde{x}>-c+\tau\} \cap M$, $c$ can be taken uniform in $\tau$ for $\tau$ in a compact set on which the strict concavity assumption on level sets of $\tilde{x}$ holds.

Remark 4.16. Notice that the proof below gives in particular, by composing $L$ and $I, L I: e^{\digamma / x} H_{\mathrm{sc}}^{s, r}(X) \rightarrow e^{\digamma / x} H_{\mathrm{sc}}^{s, r-1-s}(X), s \geq 0$, even though Proposition 3.1 implies the mapping property $L I: e^{\digamma / x} H_{\mathrm{sc}}^{s, r}(X) \rightarrow e^{\digamma / x} H_{\mathrm{sc}}^{s+1, r}(X)$ (with values in scattering one-forms or 2-tensors). The loss in the derivatives by one order and of the decay by order $\geq 1$ is due to the non-sharp treatment of the scattering Fourier integral operators $L, I$ below.

Proof. Given 4.20, we just need to show that for $s \geq 0$ there exist $R_{1}, R_{2}$ such that for $k \in \mathbb{R}, L$ is bounded

$$
e^{\digamma / x} H_{\mathrm{sc}}^{s, k+R_{1}}(U) \rightarrow e^{\digamma / x} H_{\mathrm{sc}}^{s, k}(X),
$$

while $I$ is bounded

$$
e^{\digamma / x} H_{\mathrm{sc}}^{s, k+R_{2}}(X) \rightarrow e^{\digamma / x} H_{\mathrm{sc}}^{s, k}(U),
$$


with the function spaces on $X$ with values in either one forms or 2 -tensors. To see these boundedness statements, one proceeds as in [28, Section 3], prior to Proposition 3.3, though we change our point of view slightly, as we are using the 'blown-up space' ${ }^{\text {sc }} S X$ rather than $S X$ for the geodesic parameterization.

Concretely, $L$ can be written as the composition of a multiplication operator $M$, by $x \chi(\tilde{\lambda})$, resp. $x^{3} \chi(\tilde{\lambda})$, for the one-form, resp. 2-tensor, case, times $x^{-1}$ times a sc-one-form or $x^{-2}$ times a sc-2-tensor factor, with a -1 in the power of $x$ in the definition of $L$ being absorbed into the $\tilde{\lambda}$ integral, and a push-forward in which the $\tilde{\lambda}, \tilde{\omega}$ variables are integrated out. The pushforward maps $L^{2}(U)=x^{-(2 n-1+1) / 2} L_{\mathrm{sc}}^{2}(U)$ to $L^{2}(X)=x^{-(n+1) / 2} L_{\mathrm{sc}}^{2}(X)\left(L^{2}\right.$ spaces without subscripts being relative to smooth non-degenerate densities) with the weights arising from the scattering volume forms being $x^{-2 n}$, resp. $x^{-n-1}$, times a smooth volume form. Further, it commutes with multiplication by functions of $x$, so it maps $e^{\digamma / x} H_{\mathrm{sc}}^{0, k}(X)$ to $e^{\digamma / x} H_{\mathrm{sc}}^{0, k+(n-1) / 2}(X)$, and (local) lifts of scattering vector fields $x^{2} D_{x}, x D_{y_{j}}$ are still scattering vector fields so it also maps $e^{\digamma / x} H_{\mathrm{sc}}^{s, k}(U)$ to $e^{\digamma / x} H_{\mathrm{sc}}^{s, k+(n-1) / 2}(X)$ for $s \geq 0$ integer, and then by interpolation for $s \geq 0$. Also, taking into account the smoothness of $\chi(\tilde{\lambda})$, we see that multiplication by $x^{p} \chi(\tilde{\lambda})$ maps $e^{\digamma / x} H_{\mathrm{sc}}^{s, k}(U) \rightarrow e^{\digamma / x} H_{\mathrm{sc}}^{s, k+p}(U)$ for all $s \geq 0$, so in the one form case

$$
L: e^{\digamma / x} H_{\mathrm{sc}}^{s, k}(U) \rightarrow e^{\digamma / x} H_{\mathrm{sc}}^{s, k+(n-1) / 2}(X),
$$

while in the 2 -tensor case

$$
L: e^{\digamma / x} H_{\mathrm{sc}}^{s, k}(U) \rightarrow e^{\digamma / x} H_{\mathrm{sc}}^{s, k+1+(n-1) / 2}(X) .
$$

On the other hand, $I$ can be written as a pull-back to the subset $U \times \mathbb{R}$ of ${ }^{\mathrm{sc}} S X \times \mathbb{R}$ from $X$, after contraction with $\gamma_{x, y, x \tilde{\lambda}, \tilde{\omega}}^{\prime}(t)$, via the map $\gamma:(x, y, \tilde{\lambda}, \tilde{\omega}, t) \mapsto$ $\gamma_{x, y, x \tilde{\lambda}, \tilde{\omega}}(t)$, which has surjective differential, followed by integration over (a uniformly controlled compact subset of) the $\mathbb{R}$ factor. The integration (push-forward) maps $e^{\digamma / x} H_{\mathrm{sc}}^{s, k}(U \times \mathbb{R}) \rightarrow e^{\digamma / x} H_{\mathrm{sc}}^{s, k+1 / 2}(U)$, where the $1 / 2$ shift is due to the density defining the scattering space, as above; by the same argument as above. On the other hand, the vector $\gamma_{x, y, x \tilde{\lambda}, \tilde{\omega}}^{\prime}(t)$ is $x^{-1}$ times a scattering tangent vector, as discussed in Proposition 3.1. Thus, the boundedness of the pull-back as a map

$$
x L^{2}\left(X ;{ }^{\mathrm{sc}} T^{*} X\right) \rightarrow L^{2}(U \times \mathbb{R}) \text {, i.e. } x^{-(n-1) / 2} L_{\mathrm{sc}}^{2}(X) \rightarrow x^{-(2 n+1) / 2} L_{\mathrm{sc}}^{2}(U \times \mathbb{R}),
$$

in the one-form case, resp.

$x^{2} L^{2}\left(X ; \operatorname{Sym}^{2 \mathrm{sc}} T^{*} X\right) \rightarrow L^{2}(U \times \mathbb{R})$, i.e. $x^{-(n-3) / 2} L_{\mathrm{sc}}^{2}(X) \rightarrow x^{-(2 n+1) / 2} L_{\mathrm{sc}}^{2}(U \times \mathbb{R})$,

in the 2-tensor case, follows from the surjectivity of the differential of $\gamma$. (Concretely here this means that as for fixed $\tilde{\lambda}, \tilde{\omega}, t,(x, y) \mapsto \gamma_{x, y, \tilde{\lambda}, \tilde{\omega}}(t)=\left(x^{\prime}, y^{\prime}\right)$ is a diffeomorphism, one can rewrite the integral expressing the squared $L^{2}$-norm of the pull-back in terms of the squared $L^{2}$-norm of the original function using Fubini's theorem.) Further, the $x$ coordinate along $\gamma_{x, y, \lambda, \omega}$, denoted by $x^{\prime}$ in Proposition 3.1, satisfies $x^{\prime} \geq x-C M^{2} x^{2}$ (as $|\lambda / x| \leq M$ on $U$ ) due to [28, Equation (3.1)], which means that $e^{-\digamma / x} x^{-k} e^{\digamma / x^{\prime}}\left(x^{\prime}\right)^{k}$ is bounded on the curves as $-\digamma / x+\digamma / x^{\prime}-k \log \left(x / x^{\prime}\right)$ is bounded above (with the boundedness for $x^{\prime} \leq x$, holding thanks to the lower bound for $x^{\prime}$, being the important point; for $x^{\prime} \geq x,-\digamma / x-k \log x$ being monotone for small $x$ can be used). Thus, the mapping property

$$
e^{\digamma / x} H_{\mathrm{sc}}^{0, k}(X) \rightarrow e^{\digamma / x} H_{\mathrm{sc}}^{0, k-n / 2-1}(U \times \mathbb{R}),
$$


resp.

$$
e^{\digamma / x} H_{\mathrm{sc}}^{0, k}(X) \rightarrow e^{\digamma / x} H_{\mathrm{sc}}^{0, k-n / 2-2}(U \times \mathbb{R})
$$

follows by the same argument as the $L^{2}$ boundedness. Finally, by the chain rule, using just the smoothness of $\gamma$, we obtain that any derivative of the pull-back with respect to the standard vector fields $V \in \mathcal{V}(U)$ can be expressed in terms of linear combinations with smooth coefficients of standard derivatives (with respect to $\left.V^{\prime} \in \mathcal{V}(X)\right)$ of the original function, so in particular for $P \in \operatorname{Diff}^{s}(U \times \mathbb{R})$ and one-forms, $P f$ is controlled in $e^{\digamma / x} H_{\mathrm{sc}}^{0, k-n / 2-1}(U \times \mathbb{R})$ in terms of derivatives of order $\leq s$ of $f$ in $e^{\digamma / x} H_{\mathrm{sc}}^{0, k}(X)$, with a similar statement for 2-tensors. Now, (with the above notation) $x^{\prime} \geq c x$ for some $c>0$ (so $x / x^{\prime}$ is bounded), so that $x$ factors of derivatives like $x^{2} \partial_{x}, x \partial_{y_{j}}, x \partial_{\tilde{\lambda}}, x \partial_{\tilde{\omega}_{j}}$ being applied to the pull-back can be turned into factors of $x^{\prime}$, so we see that if $P \in \operatorname{Diff}_{\mathrm{sc}}^{s}(X)$, then $P f$ is controlled in $e^{\digamma / x} H_{\mathrm{sc}}^{0, k-n / 2-1}(U \times \mathbb{R})$ in terms of derivatives of order $\leq s$ of $f$ with respect to the vector fields $x^{\prime} \partial_{x^{\prime}} x^{\prime} \partial_{y^{\prime}}$ in $e^{\digamma / x} H_{\mathrm{sc}}^{0, k}(X)$. Note here the presence of $x^{\prime} \partial_{x^{\prime}}$ rather than $\left(x^{\prime}\right)^{2} \partial_{x^{\prime}}$, due to the fact that when one writes the pull-back as $f\left(\mathrm{X}_{x, y, x \tilde{\lambda}, \tilde{\omega}}(t), \mathrm{Y}_{x, y, x \tilde{\lambda}, \tilde{\omega}}(t)\right)$, a derivative like $x \partial_{y}$ hitting it is controllable by $\left(x^{\prime} \partial_{x^{\prime}} f\right)\left(\partial_{y} \mathrm{X}\right)$ and $\left(x^{\prime} \partial_{y^{\prime}} f\right)\left(\partial_{y} \mathrm{X}\right)$, with the first of these lacking an extra factor of $x^{\prime}$. This means that we need to have an extra decay by order $s$ to get a bounded map between the scattering spaces ( since $x^{\prime} \partial_{x^{\prime}}=\left(x^{\prime}\right)^{-1}\left(\left(x^{\prime}\right)^{2} \partial_{x^{\prime}}\right)$ ), so for $s \geq 0$ integer the mapping property

$$
e^{\digamma / x} H_{\mathrm{sc}}^{s, k}(X) \rightarrow e^{\digamma / x} H_{\mathrm{sc}}^{s, k-s-n / 2-1}(U \times \mathbb{R}),
$$

resp.

$$
e^{\digamma / x} H_{\mathrm{sc}}^{s, k}(X) \rightarrow e^{\digamma / x} H_{\mathrm{sc}}^{s, k-s-n / 2-2}(U \times \mathbb{R}),
$$

follows, and then interpolation gives this for all $s \geq 0$. Thus, in the one-form case

$$
I: e^{\digamma / x} H_{\mathrm{sc}}^{s, k}(X) \rightarrow e^{\digamma / x} H_{\mathrm{sc}}^{s, k-s-n / 2-1 / 2}(U \times \mathbb{R}),
$$

in the 2-tensor case

$$
I: e^{\digamma / x} H_{\mathrm{sc}}^{s, k}(X) \rightarrow e^{\digamma / x} H_{\mathrm{sc}}^{s, k-s-n / 2-3 / 2}(U \times \mathbb{R}),
$$

completing the proof.

If $f \in x^{r} e^{\digamma / x} L_{\mathrm{sc}}^{2}(\Omega)$ then the map $f \rightarrow I f$ factors through

$$
\mathcal{S}_{\digamma, \Omega} e^{-\digamma / x} f=e^{-\digamma / x} f-\mathcal{P}_{\digamma, \Omega} e^{-\digamma / x} f
$$

since

$$
I e^{\digamma / x} \mathcal{P}_{\digamma, \Omega} e^{-\digamma / x} f=I d^{s} e^{\digamma / x} \Delta_{\digamma, s, \Omega}^{-1} e^{\digamma / x} \delta^{s} e^{-2 \digamma / x} f=0 .
$$

By Theorem 4.15, $e^{\digamma / x} \mathcal{S}_{\digamma, \Omega} e^{-\digamma / x} f \mapsto I e^{\digamma / x} \mathcal{S}_{\digamma, \Omega} e^{-\digamma / x} f$ is injective, with a stability estimate. Since

$$
e^{\digamma / x} \mathcal{P}_{\digamma, \Omega} e^{-\digamma / x} f=d^{s} e^{\digamma / x} \Delta_{\digamma, s, \Omega}^{-1} e^{\digamma / x} \delta^{s} e^{-2 \digamma / x} f,
$$

this means that we have recovered $f$ up to a potential term, i.e. in a gauge-free manner we have:

Corollary 4.17. Let $\digamma>0$. With $\Omega=\Omega_{c}$ as in Theorem 4.15, $r$ sufficiently negative, $c>0$ small, if $f \in e^{\digamma / x} x^{r} L_{\mathrm{sc}}^{2}(\Omega)$ is a one-form then $f=u+d^{s} v$, where $v \in e^{\digamma / x} x^{r} \dot{H}_{\mathrm{sc}}^{1,0}(\Omega)$, while $u \in e^{\digamma / x} x^{r} L_{\mathrm{sc}}^{2}(\Omega)$ can be stably determined from If.

Again, replacing $\Omega_{c}=\{\tilde{x}>-c\} \cap M$ by $\Omega_{\tau, c}=\{\tau>\tilde{x}>-c+\tau\} \cap M$, can be taken uniform in $\tau$ for $\tau$ in a compact set on which the strict concavity assumption on level sets of $\tilde{x}$ holds. 
Corollary 4.18. Let $\digamma, \digamma_{0}$ be as in Theorem 4.15. With $\Omega=\Omega_{c}$ as in Theorem 4.15, $r$ sufficiently negative, $c>0$ small, if $f \in x^{r} e^{\digamma / x} L_{\mathrm{sc}}^{2}(\Omega)$ is a symmetric 2-tensor then $f=u+d^{s} v$, where $v \in e^{\digamma / x} \dot{H}_{\mathrm{sc}}^{1, r-2}(\Omega)$, while $u \in e^{\digamma / x} x^{r-2} L_{\mathrm{sc}}^{2}(\Omega)$ can be stably determined from If.

Again, replacing $\Omega_{c}=\{\tilde{x}>-c\} \cap M$ by $\Omega_{\tau, c}=\{\tau>\tilde{x}>-c+\tau\} \cap M, c$ can be taken uniform in $\tau$ for $\tau$ in a compact set on which the strict concavity assumption on level sets of $\tilde{x}$ holds.

This theorem has an easy global consequence. To state this, assume that $\tilde{x}$ is a globally defined function with level sets $\Sigma_{t}$ which are strictly concave from the super-level set for $t \in(-T, 0]$, with $\tilde{x} \leq 0$ on the manifold with boundary $M$. Then we have:

Theorem 4.19. Suppose $M$ is compact. The geodesic $X$-ray transform is injective and stable modulo potentials on the restriction of one-forms and symmetric 2-tensors $f$ to $\tilde{x}^{-1}((-T, 0])$ in the following sense. For all $\tau>-T$ there is $v \in \dot{H}_{\mathrm{loc}}^{1}\left(\tilde{x}^{-1}((\tau, 0])\right)$ such that $f-d^{s} v \in L_{\mathrm{loc}}^{2}\left(\tilde{x}^{-1}((\tau, 0])\right)$ can be stably recovered from If. Here for stability we assume that $s \geq 0, f$ is in an $H^{s}$-space, the norm on If is an $H^{s}$-norm, while the norm for $v$ is an $H^{s-1}$-norm.

Proof. For the sake of contradiction, suppose there is no $v$ as stated on $\tilde{x}^{-1}\left(\left(\tau_{0}, 0\right]\right)$ for some $0>\tau_{0}>-T$, If $=0$, and let

$$
\tau=\inf \left\{t \leq 0: \exists v_{t} \in \dot{H}_{\text {loc }}^{1}(\{\tilde{x}>t\}) \text { s.t. } f=d^{s} v_{t} \text { on }\{\tilde{x}>t\}\right\} \geq \tau_{0} .
$$

Thus, for any $\tau^{\prime}>\tau$, such as $\tau^{\prime}<\tau+c / 3, c$ as in the uniform part of Corollaries 4.17 4.18 on the levels $[\tau, 0]$, there is $v \in \dot{H}_{\text {loc }}^{1}\left(\left\{\tilde{x}>\tau^{\prime}\right\}\right)$ such that $f=d^{s} v$ on $\left\{\tilde{x}>\tau^{\prime}\right\}$. Choosing $\phi \in C^{\infty}(M)$ identically 1 near $\tilde{x} \geq \tau+2 c / 3$, supported in $\tilde{x}>\tau+c / 3, f-d^{s}(\phi v)$ is supported in $\tilde{x} \leq \tau+2 c / 3$. But then by the uniform statement of Corollaries 4.17 4.18 there exists $v^{\prime} \in \dot{H}_{\mathrm{loc}}^{1}(\{\tau-c / 3<\tilde{x} \leq \tau+2 c / 3\})$ such that $f-d^{s}(\phi v)=d^{s} v^{\prime}$ in $\tau-c / 3<\tilde{x}<\tau+2 c / 3$. Extending $v^{\prime}$ as 0 , the resulting function $\tilde{v}^{\prime} \in \dot{H}_{\mathrm{loc}}^{1}(\{\tau-c / 3<\tilde{x}\})$ and $d^{s} \tilde{v}^{\prime}$ is the extension of $d^{s} v^{\prime}$ by 0 . Thus, $f=d^{s}\left(\phi v+\tilde{v}^{\prime}\right)$, and this contradicts the choice of $\tau$, completing the proof.

The stability of the recovery follows from a similar argument: by the uniform property one can recover $f$ modulo potentials in a finite number of steps: if $c$ works uniformly on $[\tau, 0]$, at most $|\tau| / c+1$ steps are necessary.

\section{REFERENCES}

[1] Yu. E. Anikonov and V. G. Romanov. On uniqueness of determination of a form of first degree by its integrals along geodesics. J. Inverse Ill-Posed Probl., 5(6):487-490 (1998), 1997.

[2] I. N. Bernstein and M. L. Gerver. A problem of integral geometry for a family of geodesics and an inverse kinematic seismics problem. Dokl. Akad. Nauk SSSR, 243(2):302-305, 1978.

[3] Jan Boman and Eric Todd Quinto. Support theorems for real-analytic Radon transforms. Duke Math. J., 55(4):943-948, 1987.

[4] Christopher Croke. Scattering rigidity with trapped geodesics. peprint, 2012.

[5] Christopher Croke and Pillar Herreros. Lens rigidity with trapped geodesics in two dimensions. preprint, 2012. http://www.math.upenn.edu/ ccroke/dvipapers/SurfaceLensRigidity.pdf.

[6] Christopher B. Croke. Conjugacy rigidity for non-positively curved graph manifolds. Ergodic Theory Dynam. Systems, 24(3):723-733, 2004.

[7] Nurlan S. Dairbekov. Integral geometry problem for nontrapping manifolds. Inverse Problems, 22(2):431-445, 2006.

[8] G. Herglotz. Über die Elastizitaet der Erde bei Beruecksichtigung ihrer variablen Dichte. Zeitschr. für Math. Phys., 52:275-299, 1905. 
[9] Venkateswaran P. Krishnan. A support theorem for the geodesic ray transform on functions. J. Fourier Anal. Appl., 15(4):515-520, 2009.

[10] Venkateswaran P. Krishnan and Plamen Stefanov. A support theorem for the geodesic ray transform of symmetric tensor fields. Inverse Probl. Imaging, 3(3):453-464, 2009.

[11] Richard B. Melrose. Geometric scattering theory. Stanford Lectures. Cambridge University Press, Cambridge, 1995.

[12] R. G. Muhometov. On a problem of reconstructing Riemannian metrics. Sibirsk. Mat. Zh., 22(3):119-135, 237, 1981.

[13] R. G Mukhometov. On the problem of integral geometry (Russian). Math. problems of geophysics, Akad. Nauk SSSR, Sibirsk., Otdel., Vychisl., Tsentr, Novosibirsk, 6(2):212-242, 1975.

[14] Gabriel P. Paternain, Mikko Salo, and Gunther Uhlmann. Tensor tomography on surfaces. Invent. Math., 193(1):229-247, 2013.

[15] L. N. Pestov and V. A. Sharafutdinov. Integral geometry of tensor fields on a manifold of negative curvature. Sibirsk. Mat. Zh., 29(3):114-130, 221, 1988.

[16] Leonid Pestov and Gunther Uhlmann. Two dimensional compact simple Riemannian manifolds are boundary distance rigid. Ann. of Math. (2), 161(2):1093-1110, 2005.

[17] Akhil Ranjan and Hemangi Shah. Convexity of spheres in a manifold without conjugate points. Proc. Indian Acad. Sci. Math. Sci., 112(4):595-599, 2002.

[18] V. A. Sharafutdinov. Integral geometry of tensor fields. Inverse and Ill-posed Problems Series. VSP, Utrecht, 1994.

[19] V. A. Sharafutdinov. Ray transform on Riemannian manifolds, lecture notes, UW-Seattle. available at: http://www.ima.umn.edu/talks/workshops/7-16-27.2001/sharafutdinov/, 1999.

[20] V. A. Sharafutdinov. A problem in integral geometry in a nonconvex domain. Sibirsk. Mat. Zh., 43(6):1430-1442, 2002.

[21] Vladimir Sharafutdinov. Variations of Dirichlet-to-Neumann map and deformation boundary rigidity of simple 2-manifolds. J. Geom. Anal., 17(1):147-187, 2007.

[22] P Stefanov. A sharp stability estimate in tensor tomography. Journal of Physics: Conference Series, 124(1):012007, 2008.

[23] Plamen Stefanov and Gunther Uhlmann. Stability estimates for the X-ray transform of tensor fields and boundary rigidity. Duke Math. J., 123(3):445-467, 2004.

[24] Plamen Stefanov and Gunther Uhlmann. Boundary rigidity and stability for generic simple metrics. J. Amer. Math. Soc., 18(4):975-1003, 2005.

[25] Plamen Stefanov and Gunther Uhlmann. Integral geometry of tensor fields on a class of non-simple Riemannian manifolds. Amer. J. Math., 130(1):239-268, 2008.

[26] Plamen Stefanov and Gunther Uhlmann. Local lens rigidity with incomplete data for a class of non-simple Riemannian manifolds. J. Differential Geom., 82(2):383-409, 2009.

[27] Plamen Stefanov, Gunther Uhlmann, and Andras Vasy. Boundary rigidity with partial data. arxiv.1210.2084, 2013

[28] Gunther Uhlmann and Andras Vasy. The inverse problem for the local geodesic ray transform. preprint, arxiv.1210.2084.

[29] E. Wiechert and K. Zoeppritz. Über Erdbebenwellen. Nachr. Koenigl. Geselschaft Wiss. Göttingen, 4:415-549, 1907.

Department of Mathematics, Purdue University, West Lafayette, IN 47907-1395, U.S.A.

E-mail address: stefanov@math.purdue.edu

Department of Mathematics, University of Washington, Seattle, WA 98195-4350, U.S.A.

E-mail address: gunther@math.washington.edu

Department of Mathematics, Stanford University, Stanford, CA 94305-2125, U.S.A.

E-mail address: andras@math.stanford.edu 